\title{
Angewandte
}

Supporting Information

(C) Wiley-VCH 2012

69451 Weinheim, Germany

\section{Asymmetric Syntheses of 8-Oxabicyclo[3,2,1]octanes: A Cationic} Cascade Cyclization**

Bin Li, Yu-Jun Zhao, Yin-Chang Lai, and Teck-Peng Loh*

ange_201202699_sm_miscellaneous_information.pdf 


\section{Contents}

1 General information $\quad$ S2

2 General procedure for cationic cascade reaction to construct 8-oxabicyclo[3,2,1]octanes S2

3 Experimental data for 8-oxabicyclo[3,2,1]octanes $\quad$ S3

4 Experimental data for the reaction precursors $\quad \mathrm{S} 12$ 


\section{General Information}

Reagents and solvents for Mukaiyama-aldol/[1,5] hydride shift cascade reaction were purified prior to use by the following procedures:

$\mathrm{TiCl}_{4}$ solution (1.0 M in $\mathrm{CH}_{2} \mathrm{Cl}_{2}$ ) was purchased from Sigma-Aldrich and used as obtained.

$\mathrm{CH}_{2} \mathrm{Cl}_{2}$ (Reagent grade, Merck) was distilled from $\mathrm{CaH}_{2}$.

$4 \AA ̊$ molecular sieves were purchased from Alfa Aesar and activated at $250{ }^{\circ} \mathrm{C}$ for 10 hours prior to use.

Analytical thin layer chromatography (TLC) was performed using Merck 60 F254 precoated silica gel plate $(0.2$ $\mathrm{mm}$ thickness). Subsequent to elution, plates were visualized using UV radiation $(254 \mathrm{~nm})$ on Spectroline Model ENF-24061/F $254 \mathrm{~nm}$. Further visualization was possible by staining with basic solution of potassium permanganate or acidic solution of ceric molybdate, followed by heating on a hot plate. Flash chromatography was performed using Merck silica gel 60 with distilled solvents. Columns were typically packed as slurry and equilibrated with hexane prior to use.

Proton nuclear magnetic resonance $\left({ }^{1} \mathrm{H}\right.$ NMR) and carbon nuclear magnetic resonance $\left({ }^{13} \mathrm{C} N \mathrm{NR}\right)$ spectroscopy were performed on Bruker Advance 300, 400 and 500 NMR spectrometers. Chemical shifts of ${ }^{1} \mathrm{H}$ NMR spectra are reported as $\mathrm{d}$ in units of parts per million ( $\mathrm{ppm})$ downfield from $\mathrm{SiMe}_{4}(\mathrm{~d}=0.0)$ and relative to the signal of chloroform- $d(\mathrm{~d}=7.264$, singlet). Multiplicities were given as: s (singlet); $\mathrm{d}$ (doublet); t (triplet); q (quartet); $\mathrm{dd}$ (doublet of doublets); ddd (doublet of doublets of doublets); $\mathrm{m}$ (multiplets) and etc. The number of protons for a given resonance is indicated by $\mathrm{nH}$. Coupling constants are reported as $J$ values in Hz. Carbon nuclear magnetic resonance spectra $\left({ }^{13} \mathrm{C}\right.$ NMR) are reported as $\mathrm{d}$ in units of parts per million (ppm) downfield from $\mathrm{SiMe}_{4}(\mathrm{~d} 0.0)$ and relative to the signal of chloroform- $d(\mathrm{~d}=77.23$, triplet). Infrared spectra were recorded on a Shimadzu IR Prestige-21 FT-IR Spectrometer. Liquid samples were examined as film between $\mathrm{NaCl}$ salt plates. Highresolution mass spectral analysis (HRMS) was performed on Q-Tof Premier mass spectrometer (Waters Corporation).

\section{General Procedure for Cationic Cascade Reaction to Construct 8- Oxabicyclo[3,2,1]octanes}

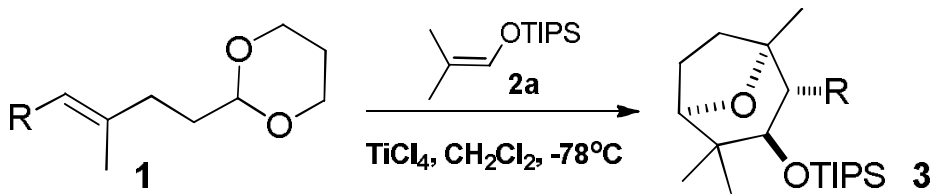

An oven-dried round bottom flask $(10 \mathrm{~mL})$ equipped with a magnetic stir bar was charged with $4 \AA$ molecular sieves $(300 \mathrm{mg})$, and sealed with a rubber septum. Then acetal $(0.20 \mathrm{mmol})$ and silyl enol ether $(0.3 \mathrm{mmol}, 1.5$ equiv) were dissolved in dry $\mathrm{CH}_{2} \mathrm{Cl}_{2}(2 \mathrm{~mL})$ and added via syringe. After cooling the solution to $-78{ }^{\circ} \mathrm{C}, \mathrm{TiCl}_{4}$ ( $0.24 \mathrm{~mL}$ of a $1.0 \mathrm{M}$ solution in $\mathrm{CH}_{2} \mathrm{Cl}_{2}, 0.24 \mathrm{mmol}, 1.2$ equiv) was added dropwisely. The solution was allowed to stir at $-78^{\circ} \mathrm{C}$ for $10 \mathrm{~h}$ and then quenched with sat. $\mathrm{NaHCO}_{3}(5 \mathrm{~mL})$, and extracted with EtOAc $(3 \mathrm{x}$ $20 \mathrm{~mL}$ ). The combined organic layers were washed with brine $(30 \mathrm{~mL})$, dried over $\mathrm{Na}_{2} \mathrm{SO}_{4}$, and concentrated in vacuo. The crude product was purified by flash column chromatography on silica gel using appropriate solvents (hexane/diethyl ether mixture) to provide the title compound. 


\section{Experimental Data for 8-Oxabicyclo[3,2,1]octanes}

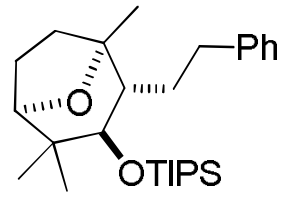

Triisopropyl((1,4,4-trimethyl-2-phenethyl-8-oxabicyclo[3.2.1] octan-3-yl)oxy)silane (3a for Table 1)

The title compound prepared following the General Procedure described above. Yield: $91 \%$ as colorless oil. ${ }^{1} \mathrm{H}$ NMR (400 MHz, $\mathrm{CDCl}_{3}$ ): d 0.93 (s, 3H), 1.04 (s, 3H), 1.07-1.10 (m, 21H), 1.38 (s, 3H), 1.51-1.64 (m, 3H), 1.81 $\left(\mathrm{ddt}, J_{l}=5.2 \mathrm{~Hz}, J_{2}=7.6 \mathrm{~Hz}, J_{3}=12.5 \mathrm{~Hz}, 1 \mathrm{H}\right), 2.11-2.14(\mathrm{~m}, 1 \mathrm{H}), 2.22-2.28(\mathrm{~m}, 1 \mathrm{H}), 2.45\left(\mathrm{dt}, J_{l}=5.1 \mathrm{~Hz}, J_{2}\right.$ $=10.0 \mathrm{~Hz}, 1 \mathrm{H}), 2.64-2.73(\mathrm{~m}, 2 \mathrm{H}), 3.67(\mathrm{~d}, J=7.9 \mathrm{~Hz}, 1 \mathrm{H}), 3.69(\mathrm{~s}, 1 \mathrm{H}), 7.16-7.20(\mathrm{~m}, 3 \mathrm{H}), 7.25-7.30$ (m, 2H); ${ }^{13} \mathrm{C}$ NMR (100 MHz, $\left.\mathrm{CDCl}_{3}\right)$ : d 12.96, 18.56, 23.88, 25.61, 26.27, 26.81, 35.29, 36.69, 37.66, 37.94, 51.23, 80.49, 80.96, 84.47, 125.90, 128.30, 128.41, 142.50. HRMS (ESI ${ }^{+}$) exact mass calcd for $\mathrm{C}_{27} \mathrm{H}_{46} \mathrm{O}_{2} \mathrm{SiNa}$ $[\mathrm{M}+\mathrm{Na}]^{+}$requires $\mathrm{m} / \mathrm{z}$ 453.3165, found $\mathrm{m} / \mathrm{z} 453.3164$.

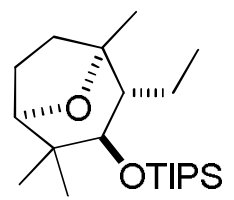

2-Ethyl-1,4,4-trimethyl-8-oxabicyclo[3.2.1]octan-3-yl)oxy)triisopropylsilane (3b for Table 1)

The title compound prepared following the General Procedure described above. Yield: $85 \%$ as colorless oil. ${ }^{1} \mathrm{H}$ NMR (400 MHz, CDCl $)$ ) d 0.93 (s, 3H), 1.00 (t, J = 7.2 Hz, 3H), 1.03 (s, 3H), 1.08-1.14 (m, 21H), 1.30 (s, 3H), $1.32-1.37(\mathrm{~m}, 1 \mathrm{H}), 1.43(\mathrm{t}, J=6.1 \mathrm{~Hz}, 1 \mathrm{H}), 1.51\left(\mathrm{dt}, J_{l}=3.8 \mathrm{~Hz}, J_{2}=12.2 \mathrm{~Hz}, 1 \mathrm{H}\right), 1.75-1.85(\mathrm{~m}, 2 \mathrm{H}), 2.24(\mathrm{dt}$, $\left.J_{l}=3.6 \mathrm{~Hz}, J_{2}=11.2 \mathrm{~Hz}, 1 \mathrm{H}\right), 2.42\left(\mathrm{dt}, J_{l}=5.2 \mathrm{~Hz}, J_{2}=10.4 \mathrm{~Hz}, 1 \mathrm{H}\right), 3.66(\mathrm{~d}, J=7.8 \mathrm{~Hz}, 1 \mathrm{H}), 3.69(\mathrm{~s}, 1 \mathrm{H})$; ${ }^{13} \mathrm{C}$ NMR (100 MHz, $\mathrm{CDCl}_{3}$ ): d 12.92, 15.47, 18.50, 23.92, 25.28, 26.22, 26.70, 36.73, 37.87, 53.13, 79.31, 80.94, 84.43. HRMS (ESI ${ }^{+}$) exact mass calcd for $\mathrm{C}_{21} \mathrm{H}_{43} \mathrm{O}_{2} \mathrm{Si}[\mathrm{M}+\mathrm{H}]^{+}$requires $\mathrm{m} / \mathrm{z} 355.3032$, found $\mathrm{m} / \mathrm{z}$ 355.3041 .

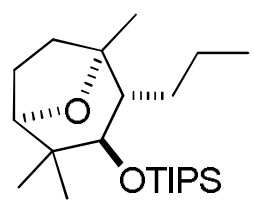

\section{Triisopropyl((1,4,4-trimethyl-2-propyl-8-oxabicyclo[3.2.1]octan-3-yl)oxy)silane (3c for Table 1)}

The title compound prepared following the General Procedure described above. Yield: $90 \%$ as colorless oil. ${ }^{1} \mathrm{H}$ NMR (400 MHz, $\left.\mathrm{CDCl}_{3}\right)$ : d $0.91(\mathrm{t}, J=7.2 \mathrm{~Hz}, 3 \mathrm{H}), 0.93$ (s, 3H), 1.04 (s, 3H), 1.05-1.14 (m, 21H), 1.18-1.26 $(\mathrm{m}, 1 \mathrm{H}), 1.28(\mathrm{~s}, 3 \mathrm{H}), 1.32-1.42(\mathrm{~m}, 2 \mathrm{H}), 1.46-1.54(\mathrm{~m}, 2 \mathrm{H}), 1.64-1.73(\mathrm{~m}, 1 \mathrm{H}), 1.79\left(\mathrm{ddt}, J_{l}=4.9 \mathrm{~Hz}, J_{2}=7.7\right.$ $\left.\mathrm{Hz}, J_{3}=12.2 \mathrm{~Hz}, 1 \mathrm{H}\right), 2.24\left(\mathrm{dt}, J_{l}=3.8 \mathrm{~Hz}, J_{2}=12.0 \mathrm{~Hz}, 1 \mathrm{H}\right), 2.41\left(\mathrm{dt}, J_{l}=4.2 \mathrm{~Hz}, J_{2}=8.8 \mathrm{~Hz}, 1 \mathrm{H}\right), 3.65(\mathrm{~d}, J$ $=6.0 \mathrm{~Hz}, 1 \mathrm{H}), 3.66(\mathrm{~s}, 1 \mathrm{H}) ;{ }^{13} \mathrm{C} \mathrm{NMR}\left(100 \mathrm{MHz}, \mathrm{CDCl}_{3}\right): \mathrm{d}$ 12.93, 14.86, 18.51, 23.91, 24.52, 25.34, 26.23, 26.79, 35.53, 36.65, 37.89, 51.62, 80.17, 80.92, 84.47. HRMS (ESI $)$ exact mass calcd for $\mathrm{C}_{22} \mathrm{H}_{45} \mathrm{O}_{2} \mathrm{Si}[\mathrm{M}+\mathrm{H}]^{+}$ requires $m / z$ 369.3189, found $m / z, 369.3202$.

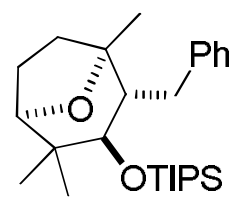

((2-Benzyl-1,4,4-trimethyl-8-oxabicyclo[3.2.1]octan-3-yl)oxy)triisopropylsilane (3d for Table 1) 
The title compound prepared following the General Procedure described above. Yield: $92 \%$ as colorless oil. ${ }^{1} \mathrm{H}$ NMR (400 MHz, $\left.\mathrm{CDCl}_{3}\right)$ : d $0.68\left(\mathrm{qd}, J_{l}=7.5 \mathrm{~Hz}, J_{2}=15.1 \mathrm{~Hz}, 3 \mathrm{H}\right), 0.89-0.93(\mathrm{~m}, 21 \mathrm{H}), 1.25(\mathrm{~s}, 3 \mathrm{H}), 1.38(\mathrm{~s}$, $3 \mathrm{H}), 1.58\left(\mathrm{dt}, J_{l}=3.7 \mathrm{~Hz}, J_{2}=12.2 \mathrm{~Hz}, 1 \mathrm{H}\right), 1.79-1.85(\mathrm{~m}, 1 \mathrm{H}), 1.89\left(\mathrm{dd}, J_{l}=5.2 \mathrm{~Hz}, J_{2}=10.8 \mathrm{~Hz}, 1 \mathrm{H}\right), 2.24$ $\left(\mathrm{dt}, J_{I}=4.0 \mathrm{~Hz}, J_{2}=12.8 \mathrm{~Hz}, 1 \mathrm{H}\right), 2.46\left(\mathrm{dt}, J_{l}=5.2 \mathrm{~Hz}, J_{2}=10.4 \mathrm{~Hz}, 1 \mathrm{H}\right), 2.64\left(\mathrm{dd}, J_{l}=10.9 \mathrm{~Hz}, J_{2}=14.0 \mathrm{~Hz}\right.$, $1 \mathrm{H}), 3.08\left(\mathrm{dd}, J_{l}=5.1 \mathrm{~Hz}, J_{2}=14.1 \mathrm{~Hz}, 1 \mathrm{H}\right), 3.59(\mathrm{~s}, 1 \mathrm{H}), 3.73(\mathrm{~d}, J=7.7 \mathrm{~Hz}, 1 \mathrm{H}), 7.15-7.19(\mathrm{~m}, 3 \mathrm{H}), 7.24-$ 7.28 (m, 2H); ${ }^{13} \mathrm{C}$ NMR (100 MHz, $\left.\mathrm{CDCl}_{3}\right)$ : d 12.44, 18.38, 18.41, 24.25, 25.83, 26.14, 28.03, 36.77, 37.03, 38.15, 52.90, 75.52, 81.01, 84.43, 125.81, 128.18, 129.62, 141.51. HRMS (ESI ${ }^{+}$) exact mass calcd for $\mathrm{C}_{26} \mathrm{H}_{45} \mathrm{O}_{2} \mathrm{Si}[\mathrm{M}+\mathrm{H}]^{+}$requires $m / z$ 417.3189, found $m / z 417.3204$.

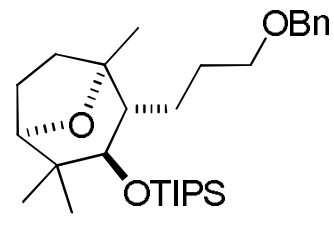

((2-(3-(Benzyloxy)propyl)-1,4,4-trimethyl-8-oxabicyclo[3.2.1]octan-3-yl)oxy)triisopropylsilane (3e for Table 1)

The title compound prepared following the General Procedure described above. Yield: $84 \%$ as colorless oil. ${ }^{1} \mathrm{H}$ NMR (400 MHz, $\mathrm{CDCl}_{3}$ ): d 0.92 (s, 3H), 1.03 (s, 3H), 1.06-1.10 (m, 21H), 1.30 (s, 3H), 1.26-1.33 (m, 1H), $1.48-1.55(\mathrm{~m}, 2 \mathrm{H}), 1.66-1.74(\mathrm{~m}, 2 \mathrm{H}), 1.75-1.85(\mathrm{~m}, 2 \mathrm{H}), 2.24\left(\mathrm{dt}, J_{l}=3.6 \mathrm{~Hz}, J_{2}=12.4 \mathrm{~Hz}, 1 \mathrm{H}\right), 2.42\left(\mathrm{dt}, J_{l}=\right.$ $\left.5.1 \mathrm{~Hz}, J_{2}=10.7 \mathrm{~Hz}, 1 \mathrm{H}\right), 3.44(\mathrm{t}, J=6.6 \mathrm{~Hz}, 2 \mathrm{H}), 3.66(\mathrm{~s}, 2 \mathrm{H}), 4.50(\mathrm{~s}, 2 \mathrm{H}), 7.27-7.34(\mathrm{~m}, 5 \mathrm{H}) ;{ }^{13} \mathrm{C} \mathrm{NMR}(100$ $\mathrm{MHz}_{\mathrm{CDCl}}$ ): d 12.92, 17.72, 18.53, 23.90, 25.41, 26.24, 26.79, 29.33, 31.46, 36.64, 37.88, 51.41, 70.73, 72.91, 80.12, 80.92, 84.48, 127.51, 127.60, 128.36, 138.58. HRMS (ESI $\left.{ }^{+}\right)$exact mass calcd for $\mathrm{C}_{29} \mathrm{H}_{51} \mathrm{O}_{3} \mathrm{Si}[\mathrm{M}+\mathrm{H}]^{+}$ requires $m / z$ 475.3607, found $m / z$ 475.3595.

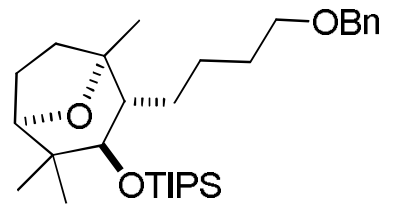

((2-(4-(Benzyloxy)butyl)-1,4,4-trimethyl-8-oxabicyclo[3.2.1]octan-3-yl)oxy)triisopropylsilane (3f for Table 1)

The title compound prepared following the General Procedure described above. Yield: $89 \%$ as colorless oil. ${ }^{1} \mathrm{H}$ NMR (400 MHz, $\mathrm{CDCl}_{3}$ ): d 0.92 (s, 3H), 1.03 (s, 3H), 1.07-1.10 (m, 21H), $1.28(\mathrm{~s}, 3 \mathrm{H}), 1.25-1.32(\mathrm{~m}, 1 \mathrm{H})$, 1.39-1.54 (m, 4H), 1.59-1.64 (m, 2H), 1.70-1.84 (m, 2H), $2.23\left(\mathrm{dt}, J_{l}=3.2 \mathrm{~Hz}, J_{2}=12.8 \mathrm{~Hz}, 1 \mathrm{H}\right), 2.41\left(\mathrm{dt}, J_{l}=\right.$ $\left.5.1 \mathrm{~Hz}, J_{2}=10.7 \mathrm{~Hz}, 1 \mathrm{H}\right), 3.47(\mathrm{t}, J=6.4 \mathrm{~Hz}, 2 \mathrm{H}), 3.66(\mathrm{~s}, 2 \mathrm{H}), 4.49(\mathrm{~s}, 2 \mathrm{H}), 7.27-7.36(\mathrm{~m}, 5 \mathrm{H}) ;{ }^{13} \mathrm{C} \mathrm{NMR}(100$ $\mathrm{MHz}_{\mathrm{CDCl}}$ ): d 12.30, 12.94, 17.72, 18.53, 23.91, 25.38, 26.23, 26.82, 27.89, 30.42, 32.84, 36.64, 37.89, 51.73, 70.12, 72.88, 80.13, 80.91, 84.48, 127.47, 127.58, 128.34, 138.67. HRMS (ESI ${ }^{+}$) exact mass calcd for $\mathrm{C}_{30} \mathrm{H}_{53} \mathrm{O}_{3} \mathrm{Si}[\mathrm{M}+\mathrm{H}]^{+}$requires $m / z$ 489.3764, found $m / z$ 489.3752.

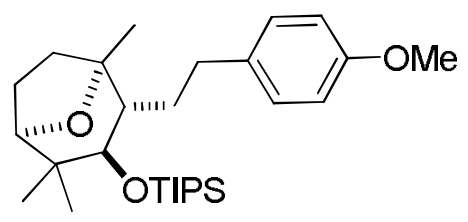

Triisopropyl((2-(4-methoxyphenethyl)-1,4,4-trimethyl-8-oxabicyclo[3.2.1]octan-3-yl)oxy)silane (3g for Table 1)

The title compound prepared following the General Procedure described above. Yield: $84 \%$ as colorless oil. ${ }^{1} \mathrm{H}$ 
NMR (400 MHz, $\mathrm{CDCl}_{3}$ ): d 0.93 (s, 3H), 1.02-1.10 (m, 24H), 1.38 (s, 3H), 1.50-1.59 (m, 3H), 1.78-1.84 (m, $1 \mathrm{H}), 2.05-2.11(\mathrm{~m}, 1 \mathrm{H}), 2.25\left(\mathrm{td}, J_{l}=3.6 \mathrm{~Hz}, J_{2}=10.0 \mathrm{~Hz}, 1 \mathrm{H}\right), 2.45\left(\mathrm{td}, J_{l}=4.8 \mathrm{~Hz}, J_{2}=10.0 \mathrm{~Hz}, 1 \mathrm{H}\right), 2.55-$ $2.62(\mathrm{~m}, 1 \mathrm{H}), 2.64-2.72(\mathrm{~m}, 1 \mathrm{H}), 3.67(\mathrm{~d}, J=7.2 \mathrm{~Hz}, 1 \mathrm{H}), 3.68(\mathrm{~s}, 1 \mathrm{H}), 3.78(\mathrm{~s}, 3 \mathrm{H}), 6.83(\mathrm{~d}, J=8.5 \mathrm{~Hz}, 2 \mathrm{H})$, $7.09(\mathrm{~d}, J=8.5 \mathrm{~Hz}, 2 \mathrm{H}) ;{ }^{13} \mathrm{C} \mathrm{NMR}\left(100 \mathrm{MHz}, \mathrm{CDCl}_{3}\right): \mathrm{d} 12.96,18.55,23.88,25.62,26.27,26.80,35.57,36.68$, 36.72, 37.93, 51.09, 55.24, 80.57, 80.96, 84.47, 113.80, 129.19, 134.60, 157.83. HRMS (ESI ${ }^{+}$) exact mass calcd for $\mathrm{C}_{28} \mathrm{H}_{49} \mathrm{O}_{3} \mathrm{Si}[\mathrm{M}+\mathrm{H}]^{+}$requires $m / z$ 461.3451, found $\mathrm{m} / \mathrm{z}$, 461.3455.

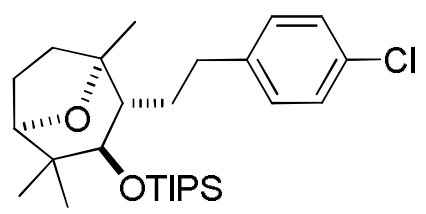

((2-(4-Chlorophenethyl)-1,4,4-trimethyl-8-oxabicyclo[3.2.1] loctan-3-yl)oxy)triisopropylsilane (3h for Table 1)

The title compound prepared following the General Procedure described above. Yield: $85 \%$ as colorless oil. ${ }^{1} \mathrm{H}$ NMR (400 MHz, $\mathrm{CDCl}_{3}$ ): d 0.93 (s, 3H), 0.99 (s, 3H), 1.10-1.10 (m, 21H), 1.37 (s, 3H), 1.50-1.59 (m, 3H), 1.77-1.86 (m, 1H), 2.04-2.14 (m, 1H), 2.21-2.28 (m, 1H), 2.41-2.48 (m, 1H), 2.58-2.74 (m, 2H), $3.66(\mathrm{~s}, 1 \mathrm{H})$, $3.67(\mathrm{~d}, J=8.2 \mathrm{~Hz}, 1 \mathrm{H}), 7.10(\mathrm{~d}, J=8.2 \mathrm{~Hz}, 2 \mathrm{H}), 7.24(\mathrm{~d}, J=8.3 \mathrm{~Hz}, 2 \mathrm{H}) ;{ }^{13} \mathrm{C} \mathrm{NMR}\left(100 \mathrm{MHz}, \mathrm{CDCl}_{3}\right): \mathrm{d}$ $12.96,18.54,23.85,25.62,26.26,26.79,35.25,36.63,36.93,37.92,51.00,80.59,80.88,84.47,128.49,129.66$, 131.63, 140.87. HRMS (ESI ${ }^{+}$) exact mass calcd for $\mathrm{C}_{27} \mathrm{H}_{46} \mathrm{ClO}_{2} \mathrm{Si}[\mathrm{M}+\mathrm{H}]^{+}$requires $\mathrm{m} / \mathrm{z}$ 465.2956, found $\mathrm{m} / \mathrm{z}$ 465.2953 .

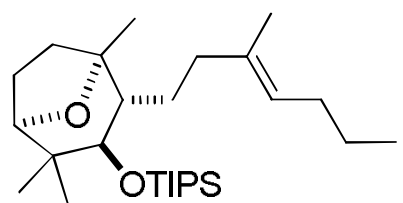

Triisopropyl((1,4,4-trimethyl-2-((E)-3-methylhept-3-en-1-yl)-8-oxabicyclo[3.2.1]octan-3-yl)oxy)silane (3i for Table 1)

The title compound prepared following the General Procedure described above. Yield: $66 \%$ as colorless oil. ${ }^{1} \mathrm{H}$ NMR (400 MHz, $\mathrm{CDCl}_{3}$ ): d 0.89 (t, $\left.J=7.4 \mathrm{~Hz}, 3 \mathrm{H}\right), 0.93$ (s, 3H), 1.05 (s, 3H), 1.09-1.11 (m, 22H), 1.28-1.38 $(\mathrm{m}, 3 \mathrm{H}), 1.31(\mathrm{~s}, 3 \mathrm{H}), 1.46-1.55(\mathrm{~m}, 2 \mathrm{H}), 1.60(\mathrm{~s}, 2 \mathrm{H}), 1.75-1.89(\mathrm{~m}, 2 \mathrm{H}), 1.95(\mathrm{q}, J=7.2 \mathrm{~Hz}, 2 \mathrm{H}), 2.02-2.06(\mathrm{~m}$, $2 \mathrm{H}), 2.21-2.27(\mathrm{~m}, 1 \mathrm{H}), 2.42\left(\mathrm{dt}, J_{l}=5.1 \mathrm{~Hz}, J_{2}=10.6 \mathrm{~Hz}, 1 \mathrm{H}\right), 3.66-3.69(\mathrm{~m}, 2 \mathrm{H}), 5.13(\mathrm{t}, J=7.1 \mathrm{~Hz}, 1 \mathrm{H}) ;{ }^{13} \mathrm{C}$ NMR (100 MHz, $\left.\mathrm{CDCl}_{3}\right)$ : d 12.95, 13.85, 16.03, 18.53, 22.96, 23.90, 25.43, 26.23, 26.80, 30.02, 31.97, 36.66, 37.90, 41.57, 51.35, 80.31, 80.98, 84.48, 124.57, 135.41. HRMS (ESI ${ }^{+}$) exact mass calcd for $\mathrm{C}_{27} \mathrm{H}_{53} \mathrm{O}_{2} \mathrm{Si}$ $[\mathrm{M}+\mathrm{H}]^{+}$requires $m / z$ 437.3815, found $m / z$ 437.3812.

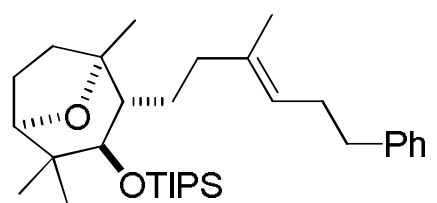

Triisopropyl((1,4,4-trimethyl-2-((E)-3-methyl-6-phenylhex-3-en-1-yl)-8-oxabicyclo[3.2.1]octan-3yl)oxy)silane (3j for Table 1$)$

The title compound prepared following the General Procedure described above. Yield: $52 \%$ as colorless oil. ${ }^{1} \mathrm{H}$ NMR (400 MHz, $\left.\mathrm{CDCl}_{3}\right)$ : d 0.93 (s, 3H), 1.02-1.11 (m, 21H), 1.05 (s, 3H), $1.30(\mathrm{~s}, 3 \mathrm{H}), 1.45-1.58(\mathrm{~m} 2 \mathrm{H}), 1.54$ $(\mathrm{s}, 3 \mathrm{H}), 1.75-1.88(\mathrm{~m}, 3 \mathrm{H}), 2.05(\mathrm{t}, J=8.4 \mathrm{~Hz}, 2 \mathrm{H}), 2.21-2.31(\mathrm{~m}, 3 \mathrm{H}), 2.38-2.45(\mathrm{~m}, 1 \mathrm{H}), 2.63(\mathrm{t}, J=7.8 \mathrm{~Hz}$, 
2H), $3.67(\mathrm{~s}, 1 \mathrm{H}), 3.68(\mathrm{~d}, J=7.9 \mathrm{~Hz}, 1 \mathrm{H}), 5.18(\mathrm{t}, J=6.8 \mathrm{~Hz}, 1 \mathrm{H}), 7.15-7.29(\mathrm{~m}, 3 \mathrm{H}), 7.26-7.29(\mathrm{~m}, 2 \mathrm{H}) ;{ }^{13} \mathrm{C}$ NMR (100 MHz, $\left.\mathrm{CDCl}_{3}\right)$ : d 12.97, 16.01, 18.55, 23.90, 25.45, 26.24, 26.82, 30.00, 31.97, 36.11, 36.67, 37.91, $41.53,51.38,80.35,80.98,84.48,123.57,125.69,128.21,128.50,136.27,142.33$. HRMS (ESI ${ }^{+}$) exact mass calcd for $\mathrm{C}_{32} \mathrm{H}_{55} \mathrm{O}_{2} \mathrm{Si}[\mathrm{M}+\mathrm{H}]^{+}$requires $m / z, 499.3971$, found $m / z, 499.3975$.

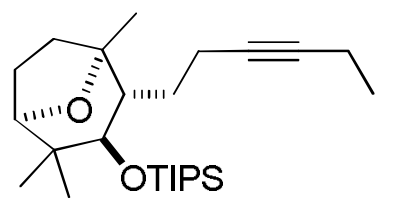

((2-(Hex-3-yn-1-yl)-1,4,4-trimethyl-8-oxabicyclo[3.2.1]octan-3-yl)oxy)triisopropylsilane (3k for Table 1) The title compound prepared following the General Procedure described above. Yield: $56 \%$ as colorless oil. ${ }^{1} \mathrm{H}$ NMR (400 MHz, CDCl $)$ ) d 0.93 (s, 3H), 1.05 (s, 3H), 1.10-1.13 (m, 24H), 1.30 (s, 3H), 1.47-1.56 (m, 3H), 1.80 (ddt, $\left.J_{1}=5.2 \mathrm{~Hz}, J_{2}=7.7 \mathrm{~Hz}, J_{3}=12.6 \mathrm{~Hz}, 1 \mathrm{H}\right), 1.93-2.03(\mathrm{~m}, 1 \mathrm{H}), 2.12-2.27(\mathrm{~m}, 5 \mathrm{H}), 2.43\left(\mathrm{ddd}, J_{l}=5.2 \mathrm{~Hz}\right.$, $\left.J_{2}=9.9 \mathrm{~Hz}, J_{3}=11.6 \mathrm{~Hz}, 1 \mathrm{H}\right), 3.63(\mathrm{~s}, 1 \mathrm{H}), 3.66(\mathrm{~d}, J=7.8 \mathrm{~Hz}, 1 \mathrm{H}) ;{ }^{13} \mathrm{C} \mathrm{NMR}\left(100 \mathrm{MHz}, \mathrm{CDCl}_{3}\right): \mathrm{d} 12.43$, 12.92, 14.26, 18.52, 19.94, 23.91, 25.38, 26.23, 26.87, 32.36, 36.61, 37.89, 50.63, 79.26, 79.83, 80.77, 81.86, 84.41. HRMS (ESI ${ }^{+}$) exact mass calcd for $\mathrm{C}_{25} \mathrm{H}_{47} \mathrm{O}_{2} \mathrm{Si}[\mathrm{M}+\mathrm{H}]^{+}$requires $m / z$ 407.3345, found $m / z$ 407.3347.

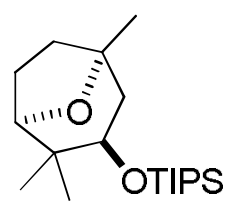

\section{Triisopropyl((1,4,4-trimethyl-8-oxabicyclo[3.2.1] octan-3-yl)oxy)silane (3l for Table 1)}

The title compound prepared following the General Procedure described above. Yield: $74 \%$ as colorless oil. ${ }^{1} \mathrm{H}$ NMR (400 MHz, CDCl $)$ : d 0.93 (s, 3H), 1.02 (s, 3H), 1.09-1.12 (m, 21H), 1.29 (s, 3H), 1.47 (ddt, $J_{I}=1.8 \mathrm{~Hz}$, $\left.J_{2}=4.4 \mathrm{~Hz}, J_{3}=12.8 \mathrm{~Hz}, 1 \mathrm{H}\right), 1.60\left(\mathrm{dd}, J_{l}=1.2 \mathrm{~Hz}, J_{2}=14.3 \mathrm{~Hz}, 1 \mathrm{H}\right), 1.82\left(\mathrm{ddt}, J_{l}=4.2 \mathrm{~Hz}, J_{2}=7.9 \mathrm{~Hz}, J_{3}=\right.$ $12.1 \mathrm{~Hz}, 1 \mathrm{H}), 1.98\left(\mathrm{ddd}, J_{l}=1.5 \mathrm{~Hz}, J_{2}=4.5 \mathrm{~Hz}, J_{3}=14.3 \mathrm{~Hz}, 1 \mathrm{H}\right), 2.22\left(\mathrm{ddd}, J_{l}=4.7 \mathrm{~Hz}, J_{2}=9.9 \mathrm{~Hz}, J_{3}=\right.$ $12.1 \mathrm{~Hz}, 1 \mathrm{H}), 2.37\left(\mathrm{ddd}, J_{l}=4.5 \mathrm{~Hz}, J_{2}=10.1 \mathrm{~Hz}, J_{3}=12.1 \mathrm{~Hz}, 1 \mathrm{H}\right), 3.68(\mathrm{~d}, J=8.1 \mathrm{~Hz}, 1 \mathrm{H}), 3.73\left(\mathrm{td}, J_{l}=1.3\right.$ $\left.\mathrm{Hz}, J_{2}=4.4 \mathrm{~Hz}, 1 \mathrm{H}\right) ;{ }^{13} \mathrm{C} \mathrm{NMR}\left(100 \mathrm{MHz}, \mathrm{CDCl}_{3}\right): \mathrm{d} 12.79,18.36,22.71,26.26,26.88,26.92,34.56,38.16$, 42.27, 74.05, 78.59, 83.74. HRMS (ESI ${ }^{+}$) exact mass calcd for $\mathrm{C}_{19} \mathrm{H}_{39} \mathrm{O}_{2} \mathrm{Si}[\mathrm{M}+\mathrm{H}]^{+}$requires $m / z$ 327.2719, found $m / z, 327.2722$.

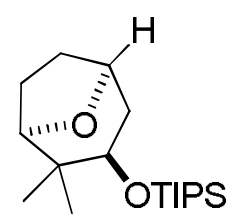

((2,2-Dimethyl-8-oxabicyclo[3.2.1]octan-3-yl)oxy)triisopropylsilane (3m for Table 1)

The title compound prepared following the General Procedure described above. Yield: $46 \%$ as colorless oil. ${ }^{1} \mathrm{H}$ NMR (400 MHz, CDCl $)$ : d 0.96 (s, 3H), 1.05 (s, 3H), 1.05-1.09 (m, 21H), 1.55 (d, J = 14.5 Hz, 1H), 1.72 (ddt, $\left.J_{I}=4.0 \mathrm{~Hz}, J_{2}=7.3 \mathrm{~Hz}, J_{3}=11.2 \mathrm{~Hz}, 1 \mathrm{H}\right), 1.81\left(\mathrm{ddt}, J_{l}=4.0 \mathrm{~Hz}, J_{2}=7.3 \mathrm{~Hz}, J_{3}=11.2 \mathrm{~Hz}, 1 \mathrm{H}\right), 2.11-2.22(\mathrm{~m}$, $2 \mathrm{H}), 2.34-2.40(\mathrm{~m}, 1 \mathrm{H}), 3.69(\mathrm{~s}, 1 \mathrm{H}), 3.69(\mathrm{~d}, J=11.9 \mathrm{~Hz}, 1 \mathrm{H}), 4.30-4.33(\mathrm{~m}, 1 \mathrm{H}) ;{ }^{13} \mathrm{C} \mathrm{NMR}(100 \mathrm{MHz}$, $\mathrm{CDCl}_{3}$ ): d 12.78, 18.36, 23.11, 24.85, 26.92, 28.34, 36.31, 39.02, 73.44, 73.79, 82.60. HRMS (ESI ${ }^{+}$) exact mass calcd for $\mathrm{C}_{18} \mathrm{H}_{37} \mathrm{O}_{2} \mathrm{Si}[\mathrm{M}+\mathrm{H}]^{+}$requires $m / z 313.2563$, found $m / z 313.2566$. 


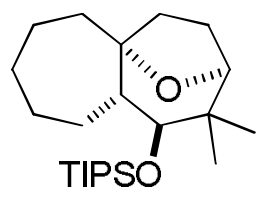

((2,2-Dimethyldecahydro-1H-3,5a-epoxyheptalen-1-yl)oxy)triisopropylsilane (3n for Table 1)

The title compound prepared following the General Procedure described above. Yield: $54 \%$ as colorless oil. ${ }^{1} \mathrm{H}$ NMR (400 MHz, $\left.\mathrm{CDCl}_{3}\right)$ : d 0.92 (s, 3H), 1.00 (s, 3H), 1.06-1.11 (m, 21H), 1.25-1.34 (m, 3H), 1.37-1.38 (m, $1 \mathrm{H}), 1.46\left(\mathrm{dt}, J_{l}=4.0 \mathrm{~Hz}, J_{2}=12.0 \mathrm{~Hz}, 1 \mathrm{H}\right), 1.65-1.73(\mathrm{~m}, 2 \mathrm{H}), 1.75-1.90(\mathrm{~m}, 6 \mathrm{H}), 2.28\left(\mathrm{dt}, J_{1}=4.1 \mathrm{~Hz}, J_{2}=\right.$ $11.2 \mathrm{~Hz}, 1 \mathrm{H}), 2.41\left(\mathrm{dt}, J_{l}=4.8 \mathrm{~Hz}, J_{2}=11.2 \mathrm{~Hz}, 1 \mathrm{H}\right), 3.54(\mathrm{~s}, 1 \mathrm{H}), 3.63(\mathrm{~d}, J=8.0 \mathrm{~Hz}, 1 \mathrm{H}) ;{ }^{13} \mathrm{C} \mathrm{NMR}(100$ $\mathrm{MHz}, \mathrm{CDCl}_{3}$ ): d 12.94, 18.46, 22.38, 23.48, 26.26, 26.42, 30.59, 30.78, 32.21, 37.39, 37.70, 41.08, 52.91, 82.76, 83.06, 83.39. HRMS $\left(\mathrm{ESI}^{+}\right)$exact mass calcd for $\mathrm{C}_{23} \mathrm{H}_{45} \mathrm{O}_{2} \mathrm{Si}[\mathrm{M}+\mathrm{H}]^{+}$requires $\mathrm{m} / z$ 381.3189, found $\mathrm{m} / \mathrm{z}$ 381.3194.<smiles>CCO[C@H]1CCC2=CCCC[C@H]2[C@@H](O[AsH])C1(C)C</smiles>

3-((8,8-Dimethyl-9-((triisopropylsilyl)oxy)-2,3,5,6,7,8,9,9a-octahydro-1H-benzo[7]annulen-7yl)oxy)propan-1-ol (3o for Table 1)

The title compound prepared following the General Procedure described above. Yield: $57 \%$ as colorless oil. ${ }^{1} \mathrm{H}$ NMR (400 MHz, $\mathrm{CDCl}_{3}$ ): d 0.84 (s, 3H), 1.11-1.16 (m, 21H), 1.16 (s, 3H), 1.36-1.57 (m, 3H), 1.70-1.91 (m, $4 \mathrm{H}), 1.97(\mathrm{~s}, 2 \mathrm{H}), 2.05-2.15(\mathrm{~m}, 2 \mathrm{H}), 2.25(\mathrm{t}, J=12.0 \mathrm{~Hz}, 1 \mathrm{H}), 2.49$ (d, $J=11.9 \mathrm{~Hz}, 1 \mathrm{H}), 2.92$ (brs, 1H), 3.53$3.60(\mathrm{~m}, 2 \mathrm{H}), 3.68(\mathrm{~s}, 1 \mathrm{H}), 3.72-3.78(\mathrm{~m}, 3 \mathrm{H}), 5.37(\mathrm{~d}, J=3.1 \mathrm{~Hz}, 1 \mathrm{H}) ;{ }^{13} \mathrm{C} \mathrm{NMR}\left(100 \mathrm{MHz}, \mathrm{CDCl}_{3}\right): \mathrm{d} 13.02$, $18.19,18.52,23.75,25.51,29.10,30.90,31.47,32.23,32.79,44.79,48.60,63.07,70.95,82.79,84.68,122.52$, 140.90. HRMS $\left(\mathrm{ESI}^{+}\right.$) exact mass calcd for $\mathrm{C}_{25} \mathrm{H}_{49} \mathrm{O}_{3} \mathrm{Si}[\mathrm{M}+\mathrm{H}]^{+}$requires $\mathrm{m} / z$ 425.3451, found $\mathrm{m} / z$ 425.3452.

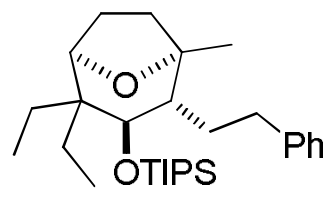

((4,4-Diethyl-1-methyl-2-phenethyl-8-oxabicyclo[3.2.1] loctan-3-yl)oxy)triisopropylsilane (3ab for Table 2) The title compound prepared following the General Procedure described above. Yield: 85\% as colorless oil. Dr: 90:10. ${ }^{1} \mathrm{H}$ NMR (400 MHz, $\left.\mathrm{CDCl}_{3}\right)$ : d $0.73(\mathrm{t}, J=7.6 \mathrm{~Hz}, 3 \mathrm{H}), 0.80$ (t, $\left.J=7.6 \mathrm{~Hz}, 3 \mathrm{H}\right), 1.03-1.14(\mathrm{~m}, 21 \mathrm{H})$, $1.38(\mathrm{~s}, 3 \mathrm{H}), 1.30-1.47(\mathrm{~m}, 2 \mathrm{H}), 1.50-1.69(\mathrm{~m}, 5 \mathrm{H}), 1.76-1.86(\mathrm{~m}, 1 \mathrm{H}), 2.06-2.15(\mathrm{~m}, 1 \mathrm{H}), 2.21-2.27(\mathrm{~m}, 1 \mathrm{H})$, 2.41-2.48 (m, 1H), 2.60-2.77 (m, $2 \mathrm{H}), 3.70(\mathrm{~s}, 0.10 \mathrm{H}$ for minor isomer), 3.80 (s, 0.90H for major isomer), 3.88 $(\mathrm{d}, J=7.77 \mathrm{~Hz}, 1 \mathrm{H}), 7.16-7.20(\mathrm{~m}, 3 \mathrm{H}), 7.25-7.30$ (m, 2H); ${ }^{13} \mathrm{C} \mathrm{NMR}\left(100 \mathrm{MHz}, \mathrm{CDCl}_{3}\right): \mathrm{d} 6.73,8.37,13.09$, 18.60, 22.30, 24.20, 25.64, 25.79, 35.20, 37.94, 41.90, 51.65, 76.82, 80.87, 81.84, 125.91, 128.31, 128.42, 142.44. HRMS $\left(\mathrm{ESI}^{+}\right)$exact mass calcd for $\mathrm{C}_{29} \mathrm{H}_{51} \mathrm{O}_{2} \mathrm{Si}[\mathrm{M}+\mathrm{H}]^{+}$requires $\mathrm{m} / \mathrm{z}$ 459.3658, found $\mathrm{m} / \mathrm{z}$, 459.3673.

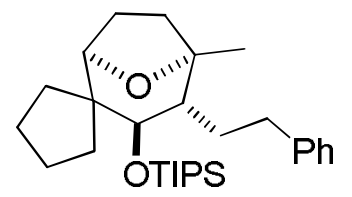

Triisopropyl((5-methyl-4-phenethyl-8-oxaspiro[bicyclo[3.2.1]octane-2,1'-cyclopentan]-3-yl)oxy)silane (3ac for Table 2) 
The title compound prepared following the General Procedure described above. Yield: $87 \%$ as colorless oil. Dr: 96:4. ${ }^{1} \mathrm{H}$ NMR (400 MHz, $\left.\mathrm{CDCl}_{3}\right)$ : d 1.02-1.13 (m, 21H), 1.36 (s, 3H), 1.26-1.41 (m, 1H), 1.48-1.66 (m, 8H), 1.77-1.91 (m, 2H), 1.97-2.11 (m, 2H), 2.22-2.29 (m, 1H), $2.52\left(\mathrm{ddd}, J_{1}=4.9 \mathrm{~Hz}, J_{2}=10.0 \mathrm{~Hz}, J_{3}=11.5 \mathrm{~Hz}\right.$, 1H), 2.63-2.73 (m, 2H), $3.77(\mathrm{~s}, 1 \mathrm{H}), 3.80(\mathrm{~d}, J=7.8 \mathrm{~Hz}, 1 \mathrm{H}), 7.16-7.20(\mathrm{~m}, 3 \mathrm{H}), 7.25-7.30(\mathrm{~m}, 2 \mathrm{H})$; ${ }^{13} \mathrm{C} \mathrm{NMR}$ (100 MHz, $\left.\mathrm{CDCl}_{3}\right)$ : d 12.95, 18.56, 23.90, 25.36, 25.72, 26.98, 33.03, 33.10, 36.50, 36.94, 37.15, 50.47, 50.99, 80.40, 81.24, 81.70, 125.88, 128.31, 128.40, 142.53. HRMS (ESI ${ }^{+}$) exact mass calcd for $\mathrm{C}_{29} \mathrm{H}_{49} \mathrm{O}_{2} \mathrm{Si}[\mathrm{M}+\mathrm{H}]^{+}$ requires $m / z$ 457.3502, found $m / z 457.3514$.<smiles>C[C@]12CC[C@@H](O1)C1(CCCCC1)[C@@H](O[Na])[C@@H]2CCc1ccccc1</smiles>

Triisopropyl((5-methyl-4-phenethyl-8-oxaspiro[bicyclo[3.2.1]octane-2,1'-cyclohexan]-3-yl)oxy)silane (3ad for Table 2)

The title compound prepared following the General Procedure described above. Yield: $91 \%$ as colorless oil. Dr: 93:7. ${ }^{1} \mathrm{H}$ NMR (400 MHz, $\left.\mathrm{CDCl}_{3}\right)$ : d 1.03-1.15 (m, 21H), 1.23-1.34 (m, 4H), 1.39 (s, 3H), 1.41-1.64 (m, 8H), 1.79-1.88 (m, 1H), 1.93 (d, $J=12.8 \mathrm{~Hz}, 1 \mathrm{H}), 2.03-2.13(\mathrm{~m}, 1 \mathrm{H}), 2.24-2.30(\mathrm{~m}, 1 \mathrm{H}), 2.42-2.49(\mathrm{~m}, 1 \mathrm{H}), 2.68$ $\left(\mathrm{dtd}, J_{l}=5.4 \mathrm{~Hz}, J_{2}=13.2 \mathrm{~Hz}, J_{3}=19.2 \mathrm{~Hz}, 2 \mathrm{H}\right), 3.68(\mathrm{~s}, 1 \mathrm{H}), 4.26(\mathrm{~d}, J=7.8 \mathrm{~Hz}, 1 \mathrm{H}), 7.16-7.20(\mathrm{~m}, 3 \mathrm{H})$, 7.26-7.30 (m, 2H); ${ }^{13} \mathrm{C}$ NMR (100 MHz, $\left.\mathrm{CDCl}_{3}\right)$ : d 13.13, 18.62, 21.46, 21.60. 25.46, 25.59, 26.44, 31.68, 33.71, 35.50, 36.90, 37.96, 39.80, 51.63, 79.25, 81.29, 81.42, 125.89, 128.30, 128.40, 142.52. HRMS (ESI ${ }^{+}$) exact mass calcd for $\mathrm{C}_{30} \mathrm{H}_{51} \mathrm{O}_{2} \mathrm{Si}[\mathrm{M}+\mathrm{H}]^{+}$requires $\mathrm{m} / \mathrm{z} 471.3658$, found $\mathrm{m} / \mathrm{z} 471.3656$.<smiles>CC12CC[C@@H](O1)[C@@H](Cc1ccccc1)[C@@H](O[Si](F)(F)F)[C@@H]2CCc1ccccc1</smiles>

4-Benzyl-1-methyl-2-phenethyl-8-oxabicyclo[3.2.1]octan-3-yl)oxy)triisopropylsilane (3ae for Table 2) The title compound prepared following the General Procedure described above. Yield: $96 \%$ as colorless oil. Dr: 59:41. ${ }^{1} \mathrm{H}$ NMR $\left(400 \mathrm{MHz}, \mathrm{CDCl}_{3}\right)$ : d 1.00-1.17 (m, 21H), $1.32(\mathrm{~s}, 1.8 \mathrm{H})$ for major isomer, $1.40(\mathrm{~s}, 1.2 \mathrm{H})$ for minor isomer, 1.44-1.67 (m, 3H), 1.71-1.83 (m, 1H), 1.88-1.99 (m, 0.6H), 2.05-2.24 (m, 2H), 2.32-2.38 (m, $0.6 \mathrm{H}), 2.52-2.61(\mathrm{~m}, 1 \mathrm{H}), 2.65-2.79(\mathrm{~m}, 3.6 \mathrm{H}), 2.85\left(\mathrm{dd}, J_{l}=10.9 \mathrm{~Hz}, J_{2}=13.0 \mathrm{~Hz}, 0.4 \mathrm{H}\right), 3.87\left(\mathrm{dd}, J_{l}=2.9 \mathrm{~Hz}\right.$, $\left.J_{2}=7.4 \mathrm{~Hz}, 0.6 \mathrm{H}\right), 3.92(\mathrm{~s}, 0.4 \mathrm{H}), 4.07(\mathrm{~d}, J=3.5 \mathrm{~Hz}, 0.6 \mathrm{H}), 4.11(\mathrm{~d}, J=7.6 \mathrm{~Hz}, 0.4 \mathrm{H}), 7.14-7.32(\mathrm{~m}, 10 \mathrm{H}) ;{ }^{13} \mathrm{C}$ NMR (100 MHz, $\left.\mathrm{CDCl}_{3}\right)$ : d 12.18, 12.89, 18.27, 18.57, 25.38, 25.58, 25.82, 30.04, 32.28, 33.43, 35.48, 36.14, 36.31, 36.46, 37.01, 38.43, 42.04, 49.27, 50.32, 51.20, 75.64, 75.80, 76.87, 80.94, 81.43, 125.93, 125.98, 126.02, 128.33, 128.38, 128.48, 128.94, 129.40, 140.19, 140.90, 142.25, 142.47. HRMS (ESI ${ }^{+}$) exact mass calcd for $\mathrm{C}_{31} \mathrm{H}_{47} \mathrm{O}_{2} \mathrm{Si}[\mathrm{M}+\mathrm{H}]^{+}$requires $\mathrm{m} / z$ 479.3345, found $\mathrm{m} / \mathrm{z} 479.3353$.

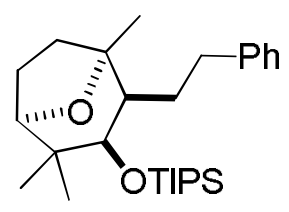

\section{Triisopropyl((1,4,4-trimethyl-2-phenethyl-8-oxabicyclo[3.2.1]octan-3-yl)oxy)silane (5a for Table 4)}

The title compound prepared following the General Procedure described above. Yield: $51 \%$ as colorless oil. Dr: 97:3. ${ }^{1} \mathrm{H}$ NMR (400 MHz, $\mathrm{CDCl}_{3}$ ): d 0.96 (s, 3H), 1.05 (s, 3H), 1.07-1.17 (m, 21H), 1.27 (d, $\left.J=12.3 \mathrm{~Hz}, 1 \mathrm{H}\right)$, $1.29(\mathrm{~s}, 3 \mathrm{H}), 1.49-1.51(\mathrm{~m}, 1 \mathrm{H}), 1.80-1.87(\mathrm{~m}, 3 \mathrm{H}), 2.28-2.35(\mathrm{~m}, 2 \mathrm{H}), 2.68-2.73(\mathrm{~m}, 2 \mathrm{H}), 3.64(\mathrm{~d}, J=8.0 \mathrm{~Hz}$, 
$1 \mathrm{H}), 3.69(\mathrm{~d}, J=2.5 \mathrm{~Hz}, 1 \mathrm{H}), 7.14-7.19(\mathrm{~m}, 3 \mathrm{H}), 7.25-7.29(\mathrm{~m}, 2 \mathrm{H}) ;{ }^{13} \mathrm{C}$ NMR $\left(100 \mathrm{MHz}, \mathrm{CDCl}_{3}\right): \mathrm{d} 14.07$, $18.81,18.82$, 23.51, 25.90, 26.97, 27.45, 29.35, 31.48, 36.33, 39.35, 46.51, 78.89, 82.74, 83.26, 125.78, 128.29, 128.31, 142.34. HRMS (ESI ${ }^{+}$) exact mass calcd for $\mathrm{C}_{27} \mathrm{H}_{47} \mathrm{O}_{2} \mathrm{Si}[\mathrm{M}+\mathrm{H}]^{+}$requires $\mathrm{m} / z 431.3345$, found $\mathrm{m} / z$ 431.3347.

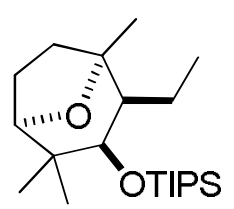

((2-Ethyl-1,4,4-trimethyl-8-oxabicyclo[3.2.1]octan-3-yl)oxy)triisopropylsilane (5b for Table 4)

The title compound prepared following the General Procedure described above. Yield: 53\% as colorless oil. Dr: 93:7. ${ }^{1} \mathrm{H}$ NMR (400 MHz, CDCl $)$ : d 0.96 (s, 3H), 0.99 (t, $\left.J=7.6 \mathrm{~Hz}, 3 \mathrm{H}\right), 1.06$ (s, 3H), 1.12-1.16 (m, 21H), 1.17-1.26 (m, 2H), $1.30(\mathrm{~s}, 3 \mathrm{H}), 1.40-1.55(\mathrm{~m}, 1 \mathrm{H}), 1.63-1.66(\mathrm{~m}, 1 \mathrm{H}), 1.76-1.87(\mathrm{~m}, 1 \mathrm{H}), 2.21-2.45(\mathrm{~m}, 2 \mathrm{H})$, $3.63(\mathrm{~d}, J=8.0 \mathrm{~Hz}, 1 \mathrm{H}), 3.70(\mathrm{~d}, J=3.4 \mathrm{~Hz}, 1 \mathrm{H}) ;{ }^{13} \mathrm{C} \mathrm{NMR}\left(100 \mathrm{MHz}, \mathrm{CDCl}_{3}\right)$ : d 12.91, 14.01, 15.07, 18.51, 18.78, 18.83, 20.49, 23.50, 25.94, 26.94, 27.50, 31.34, 39.29, 49.46, 78.63, 82.81, 83.23. HRMS (ESI ${ }^{+}$) exact mass calcd for $\mathrm{C}_{21} \mathrm{H}_{43} \mathrm{O}_{2} \mathrm{Si}[\mathrm{M}+\mathrm{H}]^{+}$requires $m / z 355.3032$, found $m / z 355.3038$.

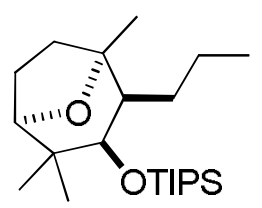

\section{Triisopropyl((1,4,4-trimethyl-2-propyl-8-oxabicyclo[3.2.1]octan-3-yl)oxy)silane (5c for Table 4)}

The title compound prepared following the General Procedure described above. Yield: 58\% as colorless oil. Dr: 93:7. ${ }^{1} \mathrm{H}$ NMR (400 MHz, $\left.\mathrm{CDCl}_{3}\right)$ : d 0.91 (t, $\left.J=7.11 \mathrm{~Hz}, 3 \mathrm{H}\right), 0.95$ (s, 3H), $1.06(\mathrm{~s}, 3 \mathrm{H}), 1.13-1.26(\mathrm{~m}, 22 \mathrm{H})$, $1.28(\mathrm{~s}, 3 \mathrm{H}), 1.33-1.51(\mathrm{~m}, 4 \mathrm{H}), 1.69-1.73(\mathrm{~m}, 1 \mathrm{H}), 1.78-1.88(\mathrm{~m}, 1 \mathrm{H}), 2.20-2.44(\mathrm{~m}, 2 \mathrm{H}), 3.63(\mathrm{~d}, J=7.1 \mathrm{~Hz}$, 1H), $3.67(\mathrm{~d}, J=4.0 \mathrm{~Hz}, 1 \mathrm{H}) ;{ }^{13} \mathrm{C}$ NMR $\left(100 \mathrm{MHz}, \mathrm{CDCl}_{3}\right): \mathrm{d} 14.05,14.46,18.51,18.75,18.83,23.54,23.60$, 25.84, 26.96, 27.50, 30.02, 31.37, 39.30, 47.12, 79.10, 82.78, 83.22. HRMS (ESI ${ }^{+}$) exact mass calcd for $\mathrm{C}_{22} \mathrm{H}_{45} \mathrm{O}_{2} \mathrm{Si}[\mathrm{M}+\mathrm{H}]^{+}$requires $\mathrm{m} / z$ 369.3189, found $m / z$ 369.3192.

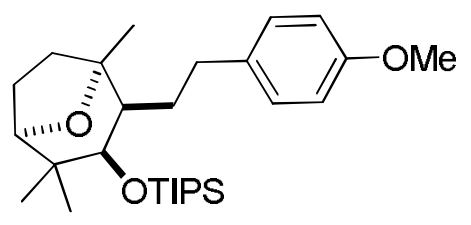

\section{Triisopropyl((2-(4-methoxyphenethyl)-1,4,4-trimethyl-8-oxabicyclo[3.2.1]octan-3-yl)oxy)silane (5d for}

\section{Table 4)}

The title compound prepared following the General Procedure described above. Yield: 56\% as colorless oil. Dr: 95:5. ${ }^{1} \mathrm{H}$ NMR (400 MHz, $\left.\mathrm{CDCl}_{3}\right)$ : d $0.95(\mathrm{~s}, 3 \mathrm{H}), 1.05(\mathrm{~s}, 3 \mathrm{H}), 1.07-1.17(\mathrm{~m}, 21 \mathrm{H}), 1.26-1.28(\mathrm{~m}, 1 \mathrm{H}), 1.30$ (s, $3 \mathrm{H}), 1.43-1.51(\mathrm{~m}, 1 \mathrm{H}), 1.76-1.87(\mathrm{~m}, 3 \mathrm{H}), 2.25-2.34(\mathrm{~m}, 2 \mathrm{H}), 2.62-2.66(\mathrm{~m}, 2 \mathrm{H}), 3.63(\mathrm{~d}, J=7.9 \mathrm{~Hz}, 1 \mathrm{H}), 3.68$ $(\mathrm{d}, J=2.6 \mathrm{~Hz}, 1 \mathrm{H}), 3.79(\mathrm{~s}, 3 \mathrm{H}), 6.82(\mathrm{~d}, J=8.6 \mathrm{~Hz}, 2 \mathrm{H}), 7.07(\mathrm{~d}, J=8.6 \mathrm{~Hz}, 2 \mathrm{H}) ;{ }^{13} \mathrm{C} \mathrm{NMR}(100 \mathrm{MHz}$, $\left.\mathrm{CDCl}_{3}\right)$ : d 14.07, 18.82, 23.51, 25.89, 26.69, 27.45, 29.51, 31.48, 35.39, 39.34, 46.43, 55.26, 78.87, 82.75, 83.26, 113.71, 129.17, 134.46, 157.70. HRMS (ESI ${ }^{+}$) exact mass calcd for $\mathrm{C}_{28} \mathrm{H}_{49} \mathrm{O}_{3} \mathrm{Si}[\mathrm{M}+\mathrm{H}]^{+}$requires $\mathrm{m} / z$ 461.3451, found $m / z, 461.3448$. 


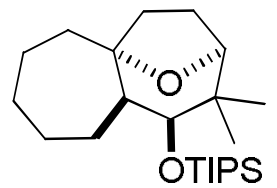

((2,2-Dimethyldecahydro-1H-3,5a-epoxyheptalen-1-yl)oxy)triisopropylsilane (5f for Table 1)

The title compound prepared following the General Procedure described above. Yield: 55\% for two isomers, $26 \%$ yield for the title compound. (another isomer data see 3n) ${ }^{1} \mathrm{H}$ NMR (400 MHz, $\left.\mathrm{CDCl}_{3}\right)$ : d 0.94 (s, $3 \mathrm{H}$ ), 1.07 (s, 3H), 1.11-1.15 (m, 22H), 1.25-1.38 (m, 5H), 1.49-1.58 (m, 1H), 1.61-1.64 (m, 1H), 1.75-1.87 (m, 4H), $1.92-$ $1.93(\mathrm{~m}, 1 \mathrm{H}), 2.27-2.32(\mathrm{~m}, 1 \mathrm{H}), 2.36-2.42(\mathrm{~m}, 1 \mathrm{H}), 3.67(\mathrm{~s}, 1 \mathrm{H}), 3.67(\mathrm{~d}, J=10.8 \mathrm{~Hz}, 1 \mathrm{H}) ;{ }^{13} \mathrm{C}$ NMR $(100$ $\mathrm{MHz}, \mathrm{CDCl}_{3}$ ): d 13.97, 18.77, 21.98, 23.07, 26.11, 27.69, 27.88, 28.04, 28.66, 31.48, 39.21, 39.61, 47.88, 80.69, 83.74, 85.02. HRMS $\left(\mathrm{ESI}^{+}\right)$exact mass calcd for $\mathrm{C}_{23} \mathrm{H}_{45} \mathrm{O}_{2} \mathrm{Si}[\mathrm{M}+\mathrm{H}]^{+}$requires $\mathrm{m} / z$ 381.3189, found $\mathrm{m} / z$ 381.3194

\section{Procedure for removing TIPS group}

To a solution of corresponding ether $(0.1 \mathrm{mmol})$ in $5 \mathrm{~mL}$ THF was added TBAF (1.0 M in THF, $0.5 \mathrm{~mL}$, 3.0 equiv) at $0{ }^{\circ} \mathrm{C}$. The reaction mixture was stirred at room temperature for $12 \mathrm{~h}$. the reaction was quenched with half saturated $\mathrm{NH}_{4} \mathrm{Cl}(5 \mathrm{~mL})$ and extracted with $\mathrm{EA}(3 \times 10 \mathrm{~mL})$. The combined organic layers were washed with brine $(25 \mathrm{~mL})$ and dried over $\mathrm{Na}_{2} \mathrm{SO}_{4}$, then concentrated in vacuo and purified by chromatography with hexane/EA as eluent gave desired alcohols in $88-94 \%$ yields

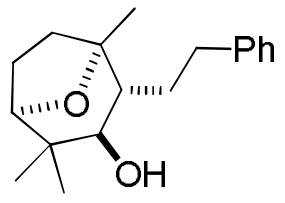

\section{1,4,4-Trimethyl-2-phenethyl-8-oxabicyclo[3.2.1]octan-3-ol (3a' for Scheme 1)}

The title compound prepared following the General Procedure described above. Yield: $94 \%$ as white solid, bp: 108-110 ${ }^{\circ} \mathrm{C} .{ }^{1} \mathrm{H}$ NMR (400 MHz, $\mathrm{CDCl}_{3}$ ): d 0.96 (s, 3H), 1.10 (s, 3H), 1.34 (s, 3H), 1.48-1.51 (m, 2H), 1.55$1.71(\mathrm{~m}, 2 \mathrm{H}), 1.84\left(\mathrm{ddt}, J_{1}=4.9 \mathrm{~Hz}, J_{2}=7.6 \mathrm{~Hz}, J_{3}=12.4 \mathrm{~Hz}, 1 \mathrm{H}\right), 2.05\left(\mathrm{ddd}, J_{1}=5.8 \mathrm{~Hz}, J_{2}=11.0 \mathrm{~Hz}, J_{3}=\right.$ $15.7 \mathrm{~Hz}, 1 \mathrm{H}), 2.17-2.32(\mathrm{~m}, 2 \mathrm{H}), 2.62-2.77(\mathrm{~m}, 2 \mathrm{H}), 3.59$ (s, 1H), $3.73(\mathrm{~d}, J=7.7 \mathrm{~Hz}, 1 \mathrm{H}), 7.17-7.19(\mathrm{~m}, 3 \mathrm{H})$, 7.26-7.31 (m, 2H); ${ }^{13} \mathrm{C}$ NMR (100 MHz, $\left.\mathrm{CDCl}_{3}\right)$ : d 22.73, 25.11, 26.09, 26.82, 33.25, 35.99, 37.26, 37.39, 76.72, 80.80, 83.83, 125.88, 128.34, 128.44, 142.33. HRMS $\left(\mathrm{ESI}^{+}\right)$exact mass calcd for $\mathrm{C}_{18} \mathrm{H}_{27} \mathrm{O}_{2}[\mathrm{M}+\mathrm{H}]^{+}$requires $m / z$ 275.2011, found $\mathrm{m} / \mathrm{z}$ 275.2003.

\section{X-ray crystal structure analysis of $3 \mathbf{a}^{\prime}$}

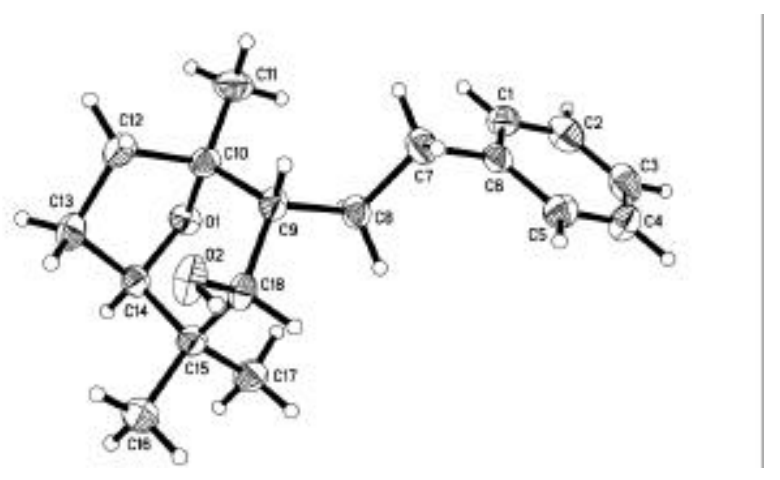

CCDC-873237 contains the supplementary crystallographic data for this paper. These data can be obtained free of charge from The Cambridge Crystallographic Data Centre via www.ccdc.cam.ac.uk/data_request/cif. 
<smiles>COc1ccc(CCC2C(O)C3(C)CCC2(C)O[C@@H]3O)cc1</smiles>

\section{2-(4-Methoxyphenethyl)-1,4,4-trimethyl-8-oxabicyclo[3.2.1]octan-3-ol (5d' for Figure 2)}

The title compound prepared following the General Procedure described above. Yield: $89 \%$ as white solid. ${ }^{1} \mathrm{H}$ NMR (400 MHz, CDCl $)_{3}$ ) d 0.98 (s, 3H), 1.05 (s, 3H), 1.26 (s, 3H), 1.29 (t, $\left.J=11.6 \mathrm{~Hz}, 1 \mathrm{H}\right), 1.40$ (d, J = 3.6 Hz, $1 \mathrm{H}), 1.58-1.66(\mathrm{~m}, 1 \mathrm{H}), 1.71-1.89(\mathrm{~m}, 3 \mathrm{H}), 2.21\left(\mathrm{dtd}, J_{l}=4.1 \mathrm{~Hz}, J_{2}=9.8 \mathrm{~Hz}, J_{3}=21.4 \mathrm{~Hz}, 2 \mathrm{H}\right), 2.45\left(\mathrm{ddd}, J_{I}\right.$ $\left.=6.5 \mathrm{~Hz}, J_{2}=9.7 \mathrm{~Hz}, J_{3}=13.6 \mathrm{~Hz}, 1 \mathrm{H}\right), 2.72\left(\mathrm{ddd}, J_{l}=5.1 \mathrm{~Hz}, J_{2}=10.5 \mathrm{~Hz}, J_{3}=13.6 \mathrm{~Hz}, 1 \mathrm{H}\right), 3.51(\mathrm{~s}, 1 \mathrm{H})$, $3.71(\mathrm{~d}, J=7.9 \mathrm{~Hz}, 1 \mathrm{H}), 3.79(\mathrm{~s}, 3 \mathrm{H}), 6.83(\mathrm{~d}, J=8.5 \mathrm{~Hz}, 2 \mathrm{H}), 7.12(\mathrm{~d}, J=8.5 \mathrm{~Hz}, 2 \mathrm{H}) ;{ }^{13} \mathrm{C} \mathrm{NMR}(100 \mathrm{MHz}$, $\left.\mathrm{CDCl}_{3}\right)$ : d 22.08, 25.54, 26.57, 26.70, 28.89, 31.21, 32.06, 38.43, 45.18, 55.28, 73.45, 82.18, 82.71, 113.82, 129.17, 134.77, 157.76. HRMS (ESI ${ }^{+}$) exact mass calcd for $\mathrm{C}_{19} \mathrm{H}_{29} \mathrm{O}_{3}[\mathrm{M}+\mathrm{H}]^{+}$requires $\mathrm{m} / \mathrm{z}$ 305.2117, found $\mathrm{m} / \mathrm{z}$ 305.2130 .

\section{X-ray crystal structure analysis of 5d'}

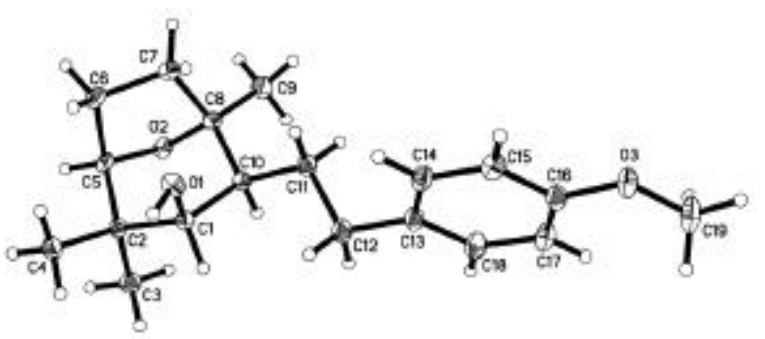

CCDC-873238 contains the supplementary crystallographic data for this paper. These data can be obtained free of charge from The Cambridge Crystallographic Data Centre via www.ccdc.cam.ac.uk/data_request/cif.

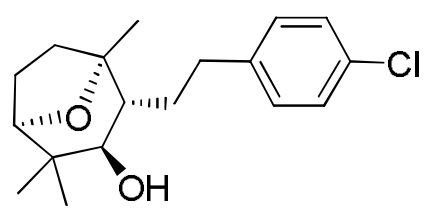

\section{(1S,2R,3R,5R)-2-(4-chlorophenethyl)-1,4,4-trimethyl-8-oxabicyclo[3.2.1]octan-3-ol (3h')}

The title compound prepared following the General Procedure described above. Yield: $88 \%$ as white solid, bp: 112-114 ${ }^{\circ} \mathrm{C} .{ }^{1} \mathrm{H}$ NMR $\left(400 \mathrm{MHz}, \mathrm{CDCl}_{3}\right.$ ): d 0.95 (s, 3H), 1.09 (s, 3H), 1.33 (s, 3H), 1.46-1.50 (m, 2H), 1.55$1.69(\mathrm{~m}, 2 \mathrm{H}), 1.84\left(\mathrm{ddt}, J_{l}=4.8 \mathrm{~Hz}, J_{2}=7.7 \mathrm{~Hz}, J_{3}=12.6 \mathrm{~Hz}, 1 \mathrm{H}\right), 2.01\left(\mathrm{ddd}, J_{l}=5.8 \mathrm{~Hz}, J_{2}=10.9 \mathrm{~Hz}, J_{3}=\right.$ $15.8 \mathrm{~Hz}, 1 \mathrm{H}), 2.24\left(\mathrm{dtd}, J_{l}=4.3 \mathrm{~Hz}, J_{2}=9.9 \mathrm{~Hz}, J_{3}=13.2 \mathrm{~Hz}, 2 \mathrm{H}\right), 2.58-2.73(\mathrm{~m}, 2 \mathrm{H}), 3.55(\mathrm{~s}, 1 \mathrm{H}), 3.73(\mathrm{~d}, J=$ $7.7 \mathrm{~Hz}, 1 \mathrm{H}), 7.11(\mathrm{~d}, J=8.3 \mathrm{~Hz}, 2 \mathrm{H}), 7.25(\mathrm{~d}, J=8.3 \mathrm{~Hz}, 2 \mathrm{H}) ;{ }^{13} \mathrm{C} \mathrm{NMR}\left(100 \mathrm{MHz}, \mathrm{CDCl}_{3}\right): \mathrm{d} 22.70,25.11$, $26.08,26.81,33.23,35.31,37.21,37.39,50.49,77.17,80.73,83.82,128.53,129.67,131.57,140.73$. HRMS $\left(\mathrm{ESI}^{+}\right.$) exact mass calcd for $\mathrm{C}_{18} \mathrm{H}_{26} \mathrm{ClO}_{2}[\mathrm{M}+\mathrm{H}]^{+}$requires $\mathrm{m} / z$ 309.1621, found $\mathrm{m} / z$ 309.1611.

\section{$\mathrm{X}$-ray crystal structure analysis of $3 \mathrm{~h}$ '}




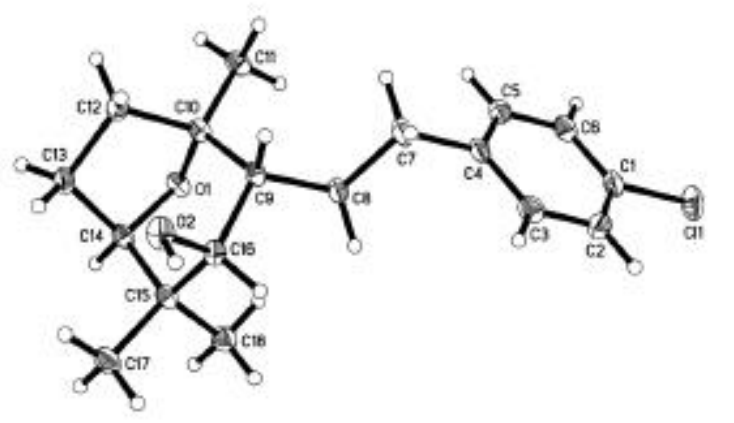

CCDC-873239 contains the supplementary crystallographic data for this paper. These data can be obtained free of charge from The Cambridge Crystallographic Data Centre via www.ccdc.cam.ac.uk/data request/cif.

\section{Experimental Data for Reaction Precursors}

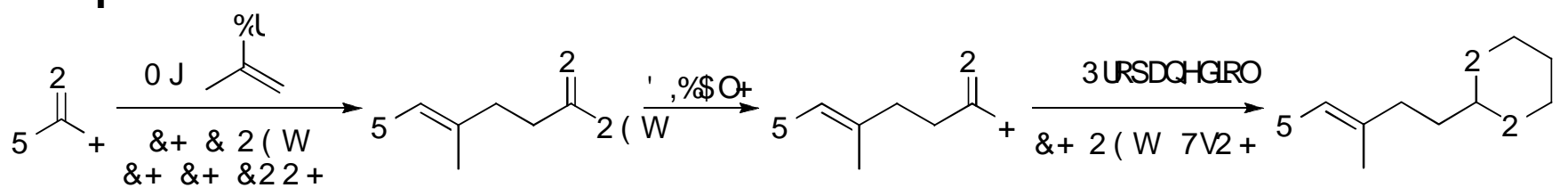

To a solution of corresponding aldehyde $(30 \mathrm{mmol})$ in THF $(30 \mathrm{~mL})$ was added 2-propenylmagnesium bromide (1.0 M in THF, $45 \mathrm{~mL}$ ) dropwise at $0{ }^{\circ} \mathrm{C}$. The mixture was stirred at room temperature for 30 mins, followed by quenching with saturated $\mathrm{NH}_{4} \mathrm{Cl}$ solution $(50 \mathrm{~mL})$. The ultimate reaction mixture was extracted with $\mathrm{Et}_{2} \mathrm{O}(2 \times 100 \mathrm{~mL})$, washed with brine $(100 \mathrm{~mL})$, dried over anhydrous $\mathrm{MgSO}_{4}$, concentrated in vacuo provided the allylic alcohols as colorless oil.

A solution of the allylic alcohol $(30 \mathrm{mmol})$ and propanoic acid $(1 \mathrm{~mL})$ in triethyl orthoacetate $(50 \mathrm{~mL})$ was heated at $145^{\circ} \mathrm{C}$ for 2 hours and enthanol was then distilled out. The reaction was cooled to room temperature and washed with saturated $\mathrm{NaHCO}_{3}$ solution $(100 \mathrm{~mL})$ and brine $(100 \mathrm{~mL})$. The organic layer was dried over $\mathrm{MgSO}_{4}$ and concentrated in vacuo to yield the ester as colourless oil in 85-96\% yields.

To a solution of the above prepared esters $(10 \mathrm{mmol})$ in $25 \mathrm{~mL}$ dry dichloromethane was added DIBAL-H ( $1 \mathrm{M}$ in heptane, $12 \mathrm{~mL}, 1.2$ equiv) dropwise at $-78^{\circ} \mathrm{C}$. The mixture was stirred at that temperature for $2.5 \mathrm{~h}$, then quenched by adding $\mathrm{MeOH}(5 \mathrm{~mL})$ and saturated aqueous solution of Rochelle's salt $(15 \mathrm{~mL})$. The resulting mixture was stirred vigorously until two clear layers were obtained (about $2 \mathrm{~h}$ ) at room temperature. The two layers were separated and the aqueous layer was extracted with $\mathrm{Et}_{2} \mathrm{O}(3 \times 20 \mathrm{~mL})$. The combined organic layers were washed with brine $(40 \mathrm{~mL})$, dried over $\mathrm{MgSO}_{4}$, filtered, and concentrated in vacuo to obtain crude aldehydes. Flash column chromatography with hexane/EA as the eluent afforded the desired products as colorless oil in $75-86 \%$ yields.

To a solution of aldehyde ( $2 \mathrm{mmol})$, TsOH ( 0.10 equiv) and 1,3-propanediol in $15 \mathrm{~mL}$ toluene was added triethylorthoformate $\left(6 \mathrm{mmol}, 3.0\right.$ equiv) at $0{ }^{\circ} \mathrm{C}$. The reaction mixture was stirred for $12 \mathrm{~h}$ at room temperature. The reaction was then quenched with triethyl amine $(0.5 \mathrm{~mL})$ and concentrated in vacuo. The crude product was purified by flash column chromatography with hexane/EA as the eluent provided the desired products as colorless oil in $78-93 \%$ yields. 
<smiles>CC(=CCCc1ccccc1)CCC1OCCCO1</smiles>

(E)-2-(3-methyl-6-phenylhex-3-en-1-yl)-1,3-dioxane (1a for Table 1)

${ }^{1} \mathrm{H}$ NMR (400 MHz, $\left.\mathrm{CDCl}_{3}\right)$ : $1.32(\mathrm{~d}, J=13.4 \mathrm{~Hz}, 1 \mathrm{H}), 1.54(\mathrm{~s}, 3 \mathrm{H}), 1.64-1.69(\mathrm{~m}, 2 \mathrm{H}), 2.03-2.13(\mathrm{~m}, 3 \mathrm{H})$, $2.30(\mathrm{q}, J=7.4 \mathrm{~Hz}, 2 \mathrm{H}), 2.63(\mathrm{t}, J=7.7 \mathrm{~Hz}, 2 \mathrm{H}), 3.73(\mathrm{t}, J=12.2 \mathrm{~Hz}, 2 \mathrm{H}), 4.09\left(\mathrm{dd}, J_{l}=5.5 \mathrm{~Hz}, J_{2}=11.2 \mathrm{~Hz}\right.$, $2 \mathrm{H}), 4.45(\mathrm{t}, J=5.2 \mathrm{~Hz}, 1 \mathrm{H}), 5.20(\mathrm{t}, J=7.1 \mathrm{~Hz}, 1 \mathrm{H}), 7.15-7.19(\mathrm{~m}, 3 \mathrm{H}), 7.25-7.28(\mathrm{~m}, 2 \mathrm{H}) ;{ }^{13} \mathrm{C} \mathrm{NMR}(100$ $\left.\mathrm{MHz}, \mathrm{CDCl}_{3}\right)$ : d 15.95, 25.87, 29.84, 33.60, 33.83, 36.04, 66.89, 102.05, 123.85, 125.67, 128.22, 128.49, 135.07, 142.31. HRMS (ESI ${ }^{+}$) exact mass calcd for $\mathrm{C}_{17} \mathrm{H}_{25} \mathrm{O}_{2}[\mathrm{M}+\mathrm{H}]^{+}$requires $\mathrm{m} / z$ 261.1855, found $\mathrm{m} / \mathrm{z}$ 261.1852.<smiles>CCC=C(C)CCC1OCCCO1</smiles>

\section{(E)-2-(3-methylhex-3-en-1-yl)-1,3-dioxane (1b for Table 1)}

${ }^{1} \mathrm{H}$ NMR (400 MHz, CDCl$)_{3}$ ): 0.93 (t, $\left.J=7.5 \mathrm{~Hz}, 3 \mathrm{H}\right), 1.34$ (d, $\left.J=13.4 \mathrm{~Hz}, 1 \mathrm{H}\right), 1.59$ (s, 3H), 1.65-1.71 (m, $2 \mathrm{H}), 1.95-2.14(\mathrm{~m}, 5 \mathrm{H}), 3.75(\mathrm{t}, J=12.2 \mathrm{~Hz}, 2 \mathrm{H}), 4.10\left(\mathrm{dd}, J_{I}=4.9 \mathrm{~Hz}, J_{2}=11.7 \mathrm{~Hz}, 2 \mathrm{H}\right), 4.49(\mathrm{t}, J=5.2 \mathrm{~Hz}$, $1 \mathrm{H}), 5.14(\mathrm{t}, J=7.0 \mathrm{~Hz}, 1 \mathrm{H}) ;{ }^{13} \mathrm{C} \mathrm{NMR}\left(100 \mathrm{MHz}, \mathrm{CDCl}_{3}\right): \mathrm{d} 14.34,15.78,21.15,25.87,33.66,33.78,66.90$, 102.13, 126.66, 133.52. HRMS (ESI ${ }^{+}$) exact mass calcd for $\mathrm{C}_{11} \mathrm{H}_{21} \mathrm{O}_{2}[\mathrm{M}+\mathrm{H}]^{+}$requires $\mathrm{m} / \mathrm{z}$ 185.1542, found $\mathrm{m} / \mathrm{z}$ 185.1543.<smiles>CCCC=C(C)CCC1OCCCO1</smiles>

(E)-2-(3-methylhept-3-en-1-yl)-1,3-dioxane (1c for Table 1)

${ }^{1} \mathrm{H}$ NMR $\left(400 \mathrm{MHz}, \mathrm{CDCl}_{3}\right)$ : d $0.88(\mathrm{t}, J=7.3 \mathrm{~Hz}, 3 \mathrm{H}), 1.30-1.39(\mathrm{~m}, 3 \mathrm{H}), 1.59(\mathrm{~s}, 3 \mathrm{H}), 1.67-1.72(\mathrm{~m}, 2 \mathrm{H}), 1.95$ (q, $J=7.2 \mathrm{~Hz}, 2 \mathrm{H}), 2.02-2.14(\mathrm{~m}, 3 \mathrm{H}), 3.75(\mathrm{t}, J=12.3 \mathrm{~Hz}, 2 \mathrm{H}), 4.10\left(\mathrm{dd}, J_{I}=4.9 \mathrm{~Hz}, J_{2}=11.0 \mathrm{~Hz}, 2 \mathrm{H}\right), 4.49$ (t, $J=5.2 \mathrm{~Hz}, 1 \mathrm{H}), 5.15(\mathrm{t}, J=6.9 \mathrm{~Hz}, 1 \mathrm{H}) ;{ }^{13} \mathrm{C} \mathrm{NMR}\left(100 \mathrm{MHz}, \mathrm{CDCl}_{3}\right): \mathrm{d} 13.78,15.95,22.95,25.88,29.97$, 33.69, 33.89, 66.92, 102.14, 124.86, 134.21. HRMS (ESI ${ }^{+}$) exact mass calcd for $\mathrm{C}_{12} \mathrm{H}_{23} \mathrm{O}_{2}[\mathrm{M}+\mathrm{H}]^{+}$requires $\mathrm{m} / \mathrm{z}$ 199.1698, found $\mathrm{m} / \mathrm{z}, 199.1696$.<smiles>CC(=CCc1ccccc1)CCC1OCCCO1</smiles>

(E)-2-(3-methyl-5-phenylpent-3-en-1-yl)-1,3-dioxane (1d for Table 1)

${ }^{1} \mathrm{H}$ NMR (400 MHz, CDCl $)$ ): d 1.32 (d, $\left.J=13.4 \mathrm{~Hz}, 1 \mathrm{H}\right), 1.71(\mathrm{~s}, 3 \mathrm{H}), 1.73-1.75(\mathrm{~m}, 2 \mathrm{H}), 2.01-2.11(\mathrm{~m}, 1 \mathrm{H})$, $2.13(\mathrm{t}, J=7.2 \mathrm{~Hz}, 2 \mathrm{H}), 3.35(\mathrm{~d}, J=7.3 \mathrm{~Hz}, 2 \mathrm{H}), 3.72(\mathrm{t}, J=12.3 \mathrm{~Hz}, 2 \mathrm{H}), 4.08\left(\mathrm{dd}, J_{l}=4.9 \mathrm{~Hz}, J_{2}=11.2 \mathrm{~Hz}\right.$, 2H), 4.49 (t, $J=5.2 \mathrm{~Hz}, 1 \mathrm{H}), 5.37(\mathrm{t}, J=7.3 \mathrm{~Hz}, 1 \mathrm{H}), 7.16-7.17(\mathrm{~m}, 3 \mathrm{H}), 7.25-7.28(\mathrm{~m}, 2 \mathrm{H}) ;{ }^{13} \mathrm{C} \mathrm{NMR}(100$ $\left.\mathrm{MHz}, \mathrm{CDCl}_{3}\right): \mathrm{d} 16.13,25.87,33.63,33.88,34.17,66.91,102.02,123.29,125.70,128.31,128.33,135.53$, 141.69. HRMS (ESI ${ }^{+}$) exact mass calcd for $\mathrm{C}_{16} \mathrm{H}_{23} \mathrm{O}_{2}[\mathrm{M}+\mathrm{H}]^{+}$requires $\mathrm{m} / z$ 247.1698, found $\mathrm{m} / z$ 247.1692.<smiles>CC(=CCCCOc1ccccc1)CCC1OCCCO1</smiles>

(E)-2-(7-(benzyloxy)-3-methylhept-3-en-1-yl)-1,3-dioxane (1e for Table 1) 
${ }^{1} \mathrm{H}$ NMR (400 MHz, $\mathrm{CDCl}_{3}$ ): d 1.33 (d, $\left.J=13.5 \mathrm{~Hz}, 1 \mathrm{H}\right), 1.60(\mathrm{~s}, 3 \mathrm{H}), 1.65-1.71(\mathrm{~m}, 4 \mathrm{H}), 2.03-2.10(\mathrm{~m}, 5 \mathrm{H})$, $3.46(\mathrm{t}, J=6.5 \mathrm{~Hz}, 2 \mathrm{H}), 3.74(\mathrm{t}, J=12.0 \mathrm{~Hz}, 2 \mathrm{H}), 4.10\left(\mathrm{dd}, J_{l}=4.8 \mathrm{~Hz}, J_{2}=11.4 \mathrm{~Hz}, 2 \mathrm{H}\right), 4.47-4.49(\mathrm{~m}, 3 \mathrm{H})$, $5.14(\mathrm{t}, J=7.1 \mathrm{~Hz}, 1 \mathrm{H}), 7.26-7.34(\mathrm{~m}, 5 \mathrm{H}) ;{ }^{13} \mathrm{C} \mathrm{NMR}\left(100 \mathrm{MHz}, \mathrm{CDCl}_{3}\right)$ : d 15.96, 24.45, 25.87, 29.83, 33.67, 33.84, 66.92, 69.91, 72.93, 102.10, 124.11, 127.48, 127.64, 128.35, 134.86, 138.68. HRMS (ESI ${ }^{+}$) exact mass calcd for $\mathrm{C}_{19} \mathrm{H}_{29} \mathrm{O}_{3}[\mathrm{M}+\mathrm{H}]^{+}$requires $m / z$ 305.2117, found $m / z$ 305.2120.<smiles>CC(=CCCCCOCc1ccccc1)CCC1OCCCO1</smiles>

(E)-2-(8-(benzyloxy)-3-methyloct-3-en-1-yl)-1,3-dioxane (1f for Table 1)

${ }^{1} \mathrm{H}$ NMR (400 MHz, $\left.\mathrm{CDCl}_{3}\right): \mathrm{d} 1.31(\mathrm{~d}, J=13.4 \mathrm{~Hz}, 1 \mathrm{H}), 1.37-1.44(\mathrm{~m}, 2 \mathrm{H}), 1.58(\mathrm{~s}, 3 \mathrm{H}), 1.59-1.63(\mathrm{~m}, 2 \mathrm{H})$, $1.66-1.71(\mathrm{~m}, 2 \mathrm{H}), 1.99(\mathrm{q}, J=7.2 \mathrm{~Hz}, 2 \mathrm{H}), 2.02-2.13(\mathrm{~m}, 1 \mathrm{H}), 2.05(\mathrm{t}, J=8.0 \mathrm{~Hz}, 2 \mathrm{H}), 3.46(\mathrm{t}, J=6.5 \mathrm{~Hz}, 2 \mathrm{H})$, $3.74(\mathrm{t}, J=12.0 \mathrm{~Hz}, 2 \mathrm{H}), 4.09\left(\mathrm{dd}, J_{l}=4.8 \mathrm{~Hz}, J_{2}=11.4 \mathrm{~Hz}, 2 \mathrm{H}\right), 4.48(\mathrm{t}, J=5.2 \mathrm{~Hz}, 1 \mathrm{H}), 4.49(\mathrm{~s}, 2 \mathrm{H}), 5.14(\mathrm{t}$, $J=7.0 \mathrm{~Hz}, 1 \mathrm{H}), 7.25-7.34(\mathrm{~m}, 5 \mathrm{H}) ;{ }^{13} \mathrm{C} \mathrm{NMR}\left(100 \mathrm{MHz}, \mathrm{CDCl}_{3}\right): \mathrm{d}$ 15.98, 25.88, 26.36, 27.66, 29.37, 33.67, 33.87, 66.91, 70.41, 72.88, 102.10, 124.66, 127.47, 127.63, 128.35, 134.42, 138.71. HRMS (ESI ${ }^{+}$) exact mass calcd for $\mathrm{C}_{20} \mathrm{H}_{31} \mathrm{O}_{3}[\mathrm{M}+\mathrm{H}]^{+}$requires $m / z$ 319.2273, found $m / z$ 319.2276.<smiles>COc1ccc(CC/C=C(\C)CCC2OCCCO2)cc1</smiles>

(E)-2-(6-(4-methoxyphenyl)-3-methylhex-3-en-1-yl)-1,3-dioxane (1g for Table 1)

${ }^{1} \mathrm{H}$ NMR (400 MHz, $\mathrm{CDCl}_{3}$ ): d 1.33 (d, $\left.J=13.4 \mathrm{~Hz}, 1 \mathrm{H}\right), 1.54(\mathrm{~s}, 3 \mathrm{H}), 1.64-1.70(\mathrm{~m}, 2 \mathrm{H}), 2.01-2.13(\mathrm{~m}, 3 \mathrm{H})$, $2.27(\mathrm{q}, J=7.4 \mathrm{~Hz}, 2 \mathrm{H}), 2.57(\mathrm{t}, J=7.7 \mathrm{~Hz}, 2 \mathrm{H}), 3.73(\mathrm{t}, J=12.2 \mathrm{~Hz}, 2 \mathrm{H}), 3.79(\mathrm{~s}, 3 \mathrm{H}), 4.09\left(\mathrm{dd}, J_{l}=4.9 \mathrm{~Hz}, J_{2}\right.$ $=11.6 \mathrm{~Hz}, 2 \mathrm{H}), 4.46(\mathrm{t}, J=5.2 \mathrm{~Hz}, 1 \mathrm{H}), 5.18(\mathrm{t}, J=7.1 \mathrm{~Hz}, 1 \mathrm{H}), 6.81(\mathrm{~d}, J=8.4 \mathrm{~Hz}, 2 \mathrm{H}), 7.09(\mathrm{~d}, J=8.4 \mathrm{~Hz}$, $2 \mathrm{H}) ;{ }^{13} \mathrm{C} \mathrm{NMR}\left(100 \mathrm{MHz}, \mathrm{CDCl}_{3}\right): \mathrm{d}$ 15.97, 25.87, 30.07, 33.60, 33.82, 35.10, 55.25, 66.90, 102.06, 113.64, 123.92, 129.34, 134.43, 134.95, 157.67. HRMS (ESI ${ }^{+}$) exact mass calcd for $\mathrm{C}_{18} \mathrm{H}_{27} \mathrm{O}_{3}[\mathrm{M}+\mathrm{H}]^{+}$requires $m / z$ 291.1960 , found $\mathrm{m} / \mathrm{z} 291.1955$.<smiles>CC(=CCCc1ccc(Cl)cc1)CCC1OCCCO1</smiles>

(E)-2-(6-(4-chlorophenyl)-3-methylhex-3-en-1-yl)-1,3-dioxane (1h for Table 1)

${ }^{1} \mathrm{H}$ NMR (400 MHz, $\mathrm{CDCl}_{3}$ ): d $1.33(\mathrm{~d}, J=13.4 \mathrm{~Hz}, 1 \mathrm{H}), 1.52(\mathrm{~s}, 3 \mathrm{H}), 1.63-1.69(\mathrm{~m}, 2 \mathrm{H}), 2.04(\mathrm{t}, J=8.0 \mathrm{~Hz}$, 2H), 2.06-2.13 (m, 1H), $2.27(\mathrm{q}, J=7.4 \mathrm{~Hz}, 2 \mathrm{H}), 2.60(\mathrm{t}, J=7.6 \mathrm{~Hz}, 2 \mathrm{H}), 3.72(\mathrm{t}, J=11.9 \mathrm{~Hz}, 2 \mathrm{H}), 4.09\left(\mathrm{dd}, J_{l}\right.$ $\left.=4.8 \mathrm{~Hz}, J_{2}=11.3 \mathrm{~Hz}, 2 \mathrm{H}\right), 4.44(\mathrm{t}, J=5.2 \mathrm{~Hz}, 1 \mathrm{H}), 5.15(\mathrm{t}, J=7.0 \mathrm{~Hz}, 1 \mathrm{H}), 7.10(\mathrm{~d}, J=8.2 \mathrm{~Hz}, 2 \mathrm{H}), 7.22(\mathrm{~d}, J$ $=8.2 \mathrm{~Hz}, 2 \mathrm{H}) ;{ }^{13} \mathrm{C}$ NMR $\left(100 \mathrm{MHz}, \mathrm{CDCl}_{3}\right): \mathrm{d} 15.98,25.86,29.62,33.60,33.78,35.31,66.89,102.00,123.36$, 128.28, 129.85, 131.36, 135.45, 140.68. HRMS (ESI ${ }^{+}$) exact mass calcd for $\mathrm{C}_{17} \mathrm{H}_{24} \mathrm{ClO}_{2}[\mathrm{M}+\mathrm{H}]^{+}$requires $\mathrm{m} / z$ 295.1465 , found $m / z 295.1468$.<smiles>CCCC=C(C)CCC=C(C)CCC1OCCCO1</smiles>

2-((3E,7E)-3,7-dimethylundeca-3,7-dien-1-yl)-1,3-dioxane (1i for Table 1) 
${ }^{1} \mathrm{H}$ NMR (400 MHz, $\left.\mathrm{CDCl}_{3}\right)$ : d 0.89 (t, $\left.J=7.3 \mathrm{~Hz}, 3 \mathrm{H}\right), 1.30-1.37$ (m, 3H), $1.59(\mathrm{~s}, 6 \mathrm{H}), 1.65-1.71(\mathrm{~m}, 2 \mathrm{H})$, $1.93-2.11(\mathrm{~m}, 9 \mathrm{H}), 3.75(\mathrm{t}, J=12.4 \mathrm{~Hz}, 2 \mathrm{H}), 4.10\left(\mathrm{dd}, J_{I}=4.9 \mathrm{~Hz}, J_{2}=11.0 \mathrm{~Hz}, 2 \mathrm{H}\right), 4.48(\mathrm{t}, J=5.2 \mathrm{~Hz}, 1 \mathrm{H})$, $5.13(\mathrm{t}, J=6.9 \mathrm{~Hz}, 2 \mathrm{H}) ;{ }^{13} \mathrm{C}$ NMR $\left(100 \mathrm{MHz}, \mathrm{CDCl}_{3}\right)$ : d 13.83, 15.98, 16.00, 23.00, 25.88, 26.63, 30.02, 33.64, 33.84, 33.69, 66.91, 102.13, 124.57, 124.63, 134.12, 134.90. HRMS (ESI ${ }^{+}$) exact mass calcd for $\mathrm{C}_{17} \mathrm{H}_{31} \mathrm{O}_{2}$ $[\mathrm{M}+\mathrm{H}]^{+}$requires $\mathrm{m} / \mathrm{z} 267.2324$, found $\mathrm{m} / \mathrm{z} 267.2329$.<smiles>CC(=CCCc1ccccc1)CCCC(C)=CCCC1OCCCO1</smiles>

2-((3E,7E)-3,7-dimethyl-10-phenyldeca-3,7-dien-1-yl)-1,3-dioxane (1j for Table 1)

${ }^{1} \mathrm{H}$ NMR $\left(400 \mathrm{MHz}, \mathrm{CDCl}_{3}\right)$ : d 1.33 (d, $\left.J=13.4 \mathrm{~Hz}, 1 \mathrm{H}\right), 1.55(\mathrm{~s}, 3 \mathrm{H}), 1.59$ (s, 3H), 1.66-1.71 (m, 2H), 1.96$2.14(\mathrm{~m}, 7 \mathrm{H}), 2.30(\mathrm{q}, J=7.4 \mathrm{~Hz}, 2 \mathrm{H}), 2.63(\mathrm{t}, J=7.8 \mathrm{~Hz}, 2 \mathrm{H}), 3.74(\mathrm{t}, J=11.4 \mathrm{~Hz}, 2 \mathrm{H}), 4.10\left(\mathrm{dd}, J_{l}=4.8 \mathrm{~Hz}\right.$, $\left.J_{2}=11.4 \mathrm{~Hz}, 2 \mathrm{H}\right), 4.49(\mathrm{t}, J=5.2 \mathrm{~Hz}, 1 \mathrm{H}), 5.12(\mathrm{t}, J=6.5 \mathrm{~Hz}, 1 \mathrm{H}), 5.18(\mathrm{t}, J=6.9 \mathrm{~Hz}, 1 \mathrm{H}), 7.15-7.20(\mathrm{~m}, 3 \mathrm{H})$, 7.25-7.29 (m, 2H); ${ }^{13} \mathrm{C}$ NMR (100 MHz, $\mathrm{CDCl}_{3}$ ): d 15.99, 25.88, 26.60, 29.99, 33.70, 33.84, 36.15, 39.65, 66.92, 102.13, 123.64, 124.48, 125.65, 128.22, 128.48, 134.24, 135.73, 142.43. HRMS (ESI $\left.{ }^{+}\right)$exact mass calcd for $\mathrm{C}_{22} \mathrm{H}_{33} \mathrm{O}_{2}[\mathrm{M}+\mathrm{H}]^{+}$requires $\mathrm{m} / \mathrm{z}$ 329.2481, found $\mathrm{m} / \mathrm{z} 329.2485$.<smiles>CCC#CCCC=C(C)CCC1OCCCO1</smiles>

\section{(E)-2-(3-methyldec-3-en-7-yn-1-yl)-1,3-dioxane (1k for Table 1)}

${ }^{1} \mathrm{H}$ NMR (400 MHz, CDCl $)$ ): d 1.11 (t, $\left.J=7.3 \mathrm{~Hz}, 3 \mathrm{H}\right), 1.34(\mathrm{~d}, J=13.4 \mathrm{~Hz}, 1 \mathrm{H}), 1.62(\mathrm{~s}, 3 \mathrm{H}), 1.67-1.72(\mathrm{~m}$, $2 \mathrm{H}), 2.05-2.20(\mathrm{~m}, 9 \mathrm{H}), 3.76(\mathrm{t}, J=12.2 \mathrm{~Hz}, 2 \mathrm{H}), 4.10\left(\mathrm{dd}, J_{I}=5.0 \mathrm{~Hz}, J_{2}=10.8 \mathrm{~Hz}, 2 \mathrm{H}\right), 4.50(\mathrm{t}, J=5.2 \mathrm{~Hz}$, $1 \mathrm{H}), 5.19(\mathrm{t}, J=6.2 \mathrm{~Hz}, 1 \mathrm{H}) ;{ }^{13} \mathrm{C} \mathrm{NMR}\left(100 \mathrm{MHz}, \mathrm{CDCl}_{3}\right): \mathrm{d} 12.43,14.33,16.06,19.20,25.87,27.80,33.52$, $33.81,66.90,79.32,81.63,102.04,123.29,135.50$. HRMS (ESI ${ }^{+}$) exact mass calcd for $\mathrm{C}_{15} \mathrm{H}_{25} \mathrm{O}_{2}[\mathrm{M}+\mathrm{H}]^{+}$ requires $\mathrm{m} / \mathrm{z} 237.1855$, found $\mathrm{m} / \mathrm{z} 237.1865$.<smiles>C=C(C)CCC1OCCCO1</smiles>

\section{2-(3-Methylbut-3-en-1-yl)-1,3-dioxane (1l for Table 1)}

${ }^{1} \mathrm{H}$ NMR (400 MHz, $\mathrm{CDCl}_{3}$ ): d 1.34 (d, $\left.J=13.5 \mathrm{~Hz}, 1 \mathrm{H}\right), 1.71-1.76(\mathrm{~m}, 2 \mathrm{H}), 1.73(\mathrm{~s}, 3 \mathrm{H}), 2.02-2.14(\mathrm{~m}, 3 \mathrm{H})$, $3.76\left(\mathrm{dt}, J_{I}=2.3 \mathrm{~Hz}, J_{2}=12.3 \mathrm{~Hz}, 2 \mathrm{H}\right), 4.11\left(\mathrm{dd}, J_{l}=5.0 \mathrm{~Hz}, J_{2}=10.8 \mathrm{~Hz}, 2 \mathrm{H}\right), 4.53(\mathrm{t}, J=5.2 \mathrm{~Hz}, 1 \mathrm{H}), 4.69(\mathrm{~s}$, 1H), $4.71(\mathrm{~s}, 1 \mathrm{H}) ;{ }^{13} \mathrm{C}$ NMR (100 MHz, $\left.\mathrm{CDCl}_{3}\right)$ : d 22.55, 25.85, 31.92, 33.25, 66.91, 101.96, 109.91 145.20. HRMS (ESI ${ }^{+}$) exact mass calcd for $\mathrm{C}_{9} \mathrm{H}_{17} \mathrm{O}_{2}[\mathrm{M}+\mathrm{H}]^{+}$requires $m / z$ 157.1229, found $m / z$ 157.1223.<smiles>C=CCCC1OCCCO1</smiles>

\section{2-(But-3-en-1-yl)-1,3-dioxane (1m for Table 1)}

${ }^{1} \mathrm{H}$ NMR $\left(400 \mathrm{MHz}, \mathrm{CDCl}_{3}\right): \mathrm{d} 1.34(\mathrm{~d}, J=13.4 \mathrm{~Hz}, 1 \mathrm{H}), 1.66-1.72(\mathrm{~m}, 2 \mathrm{H}), 2.02-2.18(\mathrm{~m}, 3 \mathrm{H}), 3.76\left(\mathrm{dt}, J_{l}=\right.$ $\left.2.2 \mathrm{~Hz}, J_{2}=12.3 \mathrm{~Hz}, 2 \mathrm{H}\right), 4.11\left(\mathrm{dd}, J_{l}=5.0 \mathrm{~Hz}, J_{2}=10.9 \mathrm{~Hz}, 2 \mathrm{H}\right), 4.53(\mathrm{t}, J=5.2 \mathrm{~Hz}, 1 \mathrm{H}), 4.96(\mathrm{~d}, J=10.2 \mathrm{~Hz}$, $1 \mathrm{H}), 5.03(\mathrm{~d}, J=17.1 \mathrm{~Hz}, 1 \mathrm{H}), 5.82\left(\mathrm{tdd}, J_{l}=6.6 \mathrm{~Hz}, J_{2}=10.2 \mathrm{~Hz}, J_{3}=16.9 \mathrm{~Hz}, 1 \mathrm{H}\right) ;{ }^{13} \mathrm{C} \mathrm{NMR}(100 \mathrm{MHz}$, $\left.\mathrm{CDCl}_{3}\right)$ : d 25.84, 28.15, 34.31, 66.90, 101.73, 114.74, 138.03. HRMS $\left(\mathrm{ESI}^{+}\right)$exact mass calcd for $\mathrm{C}_{8} \mathrm{H}_{14} \mathrm{O}_{2} \mathrm{Na}$ $[\mathrm{M}+\mathrm{Na}]^{+}$requires $\mathrm{m} / \mathrm{z}$ 165.0891, found $\mathrm{m} / \mathrm{z}$ 165.0896. 
<smiles>C1=C(CCC2CCCCC2)CCCC1</smiles>

\section{2-(2-(Cyclohept-1-en-1-yl)ethyl)-1,3-dioxane (1n for Table 1)}

${ }^{1} \mathrm{H}$ NMR $\left(400 \mathrm{MHz}, \mathrm{CDCl}_{3}\right)$ : d $1.34(\mathrm{~d}, J=13.4 \mathrm{~Hz}, 1 \mathrm{H}), 1.41-1.48(\mathrm{~m}, 4 \mathrm{H}), 1.64-1.73(\mathrm{~m}, 4 \mathrm{H}), 2.03-2.10(\mathrm{~m}$, $7 \mathrm{H}), 3.75\left(\mathrm{dt}, J_{l}=2.4 \mathrm{~Hz}, J_{2}=12.4 \mathrm{~Hz}, 2 \mathrm{H}\right), 4.10\left(\mathrm{dd}, J_{l}=4.8 \mathrm{~Hz}, J_{2}=10.4 \mathrm{~Hz}, 2 \mathrm{H}\right), 4.50(\mathrm{t}, J=5.6 \mathrm{~Hz}, 1 \mathrm{H})$, $5.55(\mathrm{t}, J=10.4 \mathrm{~Hz}, 1 \mathrm{H}) ;{ }^{13} \mathrm{C}$ NMR $\left(100 \mathrm{MHz}, \mathrm{CDCl}_{3}\right): 25.87,26.83,27.34,28.28,32.71,32.77,33.75,34.38$, 66.87, 102.13, 126.02, 143.91. HRMS (ESI ${ }^{+}$) exact mass calcd for $\mathrm{C}_{13} \mathrm{H}_{23} \mathrm{O}_{2}[\mathrm{M}+\mathrm{H}]^{+}$requires $m / z 211.1698$, found $\mathrm{m} / \mathrm{z} 211.1701$.<smiles>C1=C(CCC2OCCCO2)CCCC1</smiles>

\section{2-(2-(Cyclohex-1-en-1-yl)ethyl)-1,3-dioxane (1o for Table 1)}

${ }^{1} \mathrm{H}$ NMR $\left(400 \mathrm{MHz}, \mathrm{CDCl}_{3}\right): \mathrm{d} 1.33(\mathrm{~d}, J=13.4 \mathrm{~Hz}, 1 \mathrm{H}), 1.51-1.71(\mathrm{~m}, 6 \mathrm{H}), 1.89-2.14(\mathrm{~m}, 7 \mathrm{H}), 3.75\left(\mathrm{dt}, J_{l}=\right.$ $\left.2.4 \mathrm{~Hz}, J_{2}=12.4 \mathrm{~Hz}, 2 \mathrm{H}\right), 4.10\left(\mathrm{dd}, J_{I}=5.0 \mathrm{~Hz}, J_{2}=10.7 \mathrm{~Hz}, 2 \mathrm{H}\right), 4.50(\mathrm{t}, J=5.2 \mathrm{~Hz}, 1 \mathrm{H}), 5.42(\mathrm{~s}, 1 \mathrm{H}) ;{ }^{13} \mathrm{C}$ NMR (100 MHz, $\left.\mathrm{CDCl}_{3}\right)$ : d 22.52, 22.98, 25.22, 25.86, 28.34, 32.15, 33.36, 66.89, 102.20, 120.90, 136.98 . HRMS (ESI ${ }^{+}$) exact mass calcd for $\mathrm{C}_{12} \mathrm{H}_{21} \mathrm{O}_{2}[\mathrm{M}+\mathrm{H}]^{+}$requires $\mathrm{m} / \mathrm{z}$ 197.1542, found $\mathrm{m} / \mathrm{z}$ 197.1544. General procedure for chiral acetals synthesis<smiles>[R]C=C(C)CCC(OCC)OCC</smiles>

To a solution of corresponding achiral acetal $(1 \mathrm{mmol})$ in DCM $(5 \mathrm{~mL})$ was added chiral diol $(1.2 \mathrm{mmol}$, 1.2 equiv) and $\mathrm{TsOH}(0.1 \mathrm{mmol})$ at room temperature. The mixture was stirred at room temperature for $5 \mathrm{~h}$, followed by quenching with triethyl amine $(0.1 \mathrm{~mL})$. The reaction mixture concentrated in vacuo and purified by chromatography with hexane/EA as the eluent provided the desired products as colorless oil in $85-94 \%$ yields.<smiles>CC(=CCCc1ccccc1)CCC1O[C@@H](C)C[C@@H](C)O1</smiles>

\section{(4R,6R)-4,6-dimethyl-2-((E)-3-methyl-6-phenylhex-3-en-1-yl)-1,3-dioxane (1aa for Table 3)}

Yield: $94 \%$. [a] $]_{\mathrm{D}}^{20}=16.1^{\circ}\left(c=4.60\right.$ in $\left.\mathrm{CHCl}_{3}\right) .{ }^{1} \mathrm{H}$ NMR $\left(400 \mathrm{MHz}, \mathrm{CDCl}_{3}\right): \mathrm{d} 1.20(\mathrm{~d}, J=6.1 \mathrm{~Hz}, 3 \mathrm{H}), 1.33(\mathrm{~d}$, $J=7.0 \mathrm{~Hz}, 4 \mathrm{H}), 1.55(\mathrm{~s}, 3 \mathrm{H}), 1.60-1.68(\mathrm{~m}, 2 \mathrm{H}), 1.79-1.87(\mathrm{~m}, 1 \mathrm{H}), 2.05(\mathrm{t}, J=7.9 \mathrm{~Hz}, 2 \mathrm{H}), 2.30(\mathrm{q}, J=7.4 \mathrm{~Hz}$, $2 \mathrm{H}), 2.62(\mathrm{t}, J=7.8 \mathrm{~Hz}, 2 \mathrm{H}), 3.91\left(\mathrm{dqd}, J_{l}=2.4 \mathrm{~Hz}, J_{2}=6.2 \mathrm{~Hz}, J_{3}=12.4 \mathrm{~Hz}, 1 \mathrm{H}\right), 4.29(\mathrm{p}, J=6.8 \mathrm{~Hz}, 1 \mathrm{H})$, 4.79 (t, $J=5.2 \mathrm{~Hz}, 1 \mathrm{H}), 5.20(\mathrm{t}, J=7.0 \mathrm{~Hz}, 1 \mathrm{H}), 7.15-7.19(\mathrm{~m}, 3 \mathrm{H}), 7.25-7.28(\mathrm{~m} 2 \mathrm{H}) ;{ }^{13} \mathrm{C}$ NMR $(100 \mathrm{MHz}$, $\left.\mathrm{CDCl}_{3}\right): \mathrm{d} 15.97,17.24,21.90,29.88,33.56,34.03,36.08,36.86,67.50,67.95,93.98,123.75,125.66,128.22$, 128.45, 135.16, 142.34. HRMS (ESI ${ }^{+}$) exact mass calcd for $\mathrm{C}_{19} \mathrm{H}_{29} \mathrm{O}_{2}[\mathrm{M}+\mathrm{H}]^{+}$requires $\mathrm{m} / z$ 289.2168, found $\mathrm{m} / \mathrm{z}$ 289.2176 . 
<smiles>CC(=CCCc1ccccc1)CCC1OC(c2ccccc2)C(c2ccccc2)O1</smiles>

(4R,5R)-2-((E)-3-methyl-6-phenylhex-3-en-1-yl)-4,5-diphenyl-1,3-dioxolane (1ab for Table 3) Yield: $85 \%$. $[\mathrm{a}]_{\mathrm{D}}{ }^{20}=10.0^{\circ}\left(c=1.03\right.$ in $\left.\mathrm{CHCl}_{3}\right) .{ }^{1} \mathrm{H} \mathrm{NMR}\left(400 \mathrm{MHz}, \mathrm{CDCl}_{3}\right): \mathrm{d} 1.62(\mathrm{~s}, 3 \mathrm{H}), 1.97-2.02(\mathrm{~m}, 2 \mathrm{H})$, 2.26-2.37 (m, 4H), $2.66(\mathrm{t}, J=7.8 \mathrm{~Hz}, 2 \mathrm{H}), 4.73(\mathrm{~d}, J=7.8 \mathrm{~Hz}, 1 \mathrm{H}), 4.76(\mathrm{~d}, J=7.6 \mathrm{~Hz}, 1 \mathrm{H}), 5.30(\mathrm{t}, J=7.0 \mathrm{~Hz}$, $1 \mathrm{H}), 5.50(\mathrm{t}, J=4.5 \mathrm{~Hz}, 1 \mathrm{H}), 7.18-7.35(\mathrm{~m}, 15 \mathrm{H}) ;{ }^{13} \mathrm{C} \mathrm{NMR}\left(100 \mathrm{MHz}, \mathrm{CDCl}_{3}\right): \mathrm{d}$ 16.07, 29.99, 33.14, 33.66, 36.08, 84.94, 86.81, 124.01, 125.71, 126.35, 126.81, 128.09, 128.25, 128.47, 128.50, 128.54, 128.58, 135.03, 136.95, 138.59, 142.32. HRMS (ESI ${ }^{+}$) exact mass calcd for $\mathrm{C}_{28} \mathrm{H}_{30} \mathrm{O}_{2} \mathrm{Na}[\mathrm{M}+\mathrm{Na}]^{+}$requires $m / z$ 421.2144, found $m / z 421.2151$.<smiles>CC(=CCCc1ccccc1)CCC1O[C@@H](C)[C@@H](C)O1</smiles>

(4R,5R)-4,5-dimethyl-2-((E)-3-methyl-6-phenylhex-3-en-1-yl)-1,3-dioxolane (1a' for Table 3)

Yield: $90 \%$. $[\mathrm{a}]_{\mathrm{D}}{ }^{20}=59.8^{\circ}\left(c=0.95\right.$ in $\left.\mathrm{CHCl}_{3}\right) .{ }^{1} \mathrm{H}$ NMR $\left(400 \mathrm{MHz}, \mathrm{CDCl}_{3}\right): \mathrm{d} 1.22(\mathrm{~d}, J=5.6 \mathrm{~Hz}, 3 \mathrm{H}), 1.29(\mathrm{~d}$, $J=5.6 \mathrm{~Hz}, 3 \mathrm{H}), 1.55(\mathrm{~s}, 3 \mathrm{H}), 1.69-1.74(\mathrm{~m}, 2 \mathrm{H}), 2.06-2.10(\mathrm{~m}, 2 \mathrm{H}), 2.30(\mathrm{q}, J=7.6 \mathrm{~Hz}, 2 \mathrm{H}), 2.61-2.65(\mathrm{~m}, 2 \mathrm{H})$, $3.58-3.64(\mathrm{~m}, 2 \mathrm{H}), 5.02(\mathrm{t}, J=4.7 \mathrm{~Hz}, 1 \mathrm{H}), 5.22(\mathrm{t}, J=7.0 \mathrm{~Hz}, 1 \mathrm{H}), 7.15-7.19(\mathrm{~m}, 3 \mathrm{H}), 7.25-7.28(\mathrm{~m}, 2 \mathrm{H}) ;{ }^{13} \mathrm{C}$ NMR (100 MHz, $\left.\mathrm{CDCl}_{3}\right)$ : d 16.03, 16.98, 17.31, 29.94, 33.16, 33.69, 36.06, 78.10, 79.75, 103.03, 123.66, 125.67, 128.21, 128.48, 135.11, 142.33. HRMS $\left(\mathrm{ESI}^{+}\right)$exact mass calcd for $\mathrm{C}_{18} \mathrm{H}_{27} \mathrm{O}_{2}[\mathrm{M}+\mathrm{H}]^{+}$requires $m / z$ 275.2011, found $m / z, 275.2013$.<smiles>C[C]1[Z]2C(CC/C(C)=C\Cc3ccccc3)O[C@@H]2[C@@H]1C</smiles>

(4R,5R)-4,5-dimethyl-2-((E)-3-methyl-5-phenylpent-3-en-1-yl)-1,3-dioxolane (1d' for Table 3) Yield: $87 \%$. $[\mathrm{a}]_{\mathrm{D}}{ }^{20}=-11.9^{\circ}\left(c=1.54\right.$ in $\left.\mathrm{CHCl}_{3}\right) .{ }^{1} \mathrm{H}$ NMR $\left(400 \mathrm{MHz}, \mathrm{CDCl}_{3}\right): \mathrm{d} 1.22(\mathrm{~d}, J=5.6 \mathrm{~Hz}, 3 \mathrm{H}), 1.28$ $(\mathrm{d}, J=5.6 \mathrm{~Hz}, 3 \mathrm{H}), 1.72(\mathrm{~s}, 3 \mathrm{H}), 1.75-1.80(\mathrm{~m}, 2 \mathrm{H}), 2.14-2.18(\mathrm{~m}, 2 \mathrm{H}), 3.35(\mathrm{~d}, J=7.3 \mathrm{~Hz}, 2 \mathrm{H}), 3.57-3.61(\mathrm{~m}$, 2H), $5.04(\mathrm{t}, J=4.7 \mathrm{~Hz}, 1 \mathrm{H}), 5.39(\mathrm{t}, J=7.3 \mathrm{~Hz}, 1 \mathrm{H}), 7.16-7.18(\mathrm{~m}, 3 \mathrm{H}), 7.25-7.28(\mathrm{~m}, 2 \mathrm{H}) ;{ }^{13} \mathrm{C} \mathrm{NMR}(100$ $\left.\mathrm{MHz}, \mathrm{CDCl}_{3}\right)$ : d 16.26, 16.98, 17.30, 33.19, 33.71, 34.18, 78.12, 79.76, 103.00, 123.07, 125.70, 128.31, 128.34, 135.59, 141.67. HRMS (ESI ${ }^{+}$) exact mass calcd for $\mathrm{C}_{17} \mathrm{H}_{25} \mathrm{O}_{2}[\mathrm{M}+\mathrm{H}]^{+}$requires $\mathrm{m} / \mathrm{z} 261.1855$, found $\mathrm{m} / \mathrm{z}$ 261.1866.<smiles>CC(=CCCc1ccc(Cl)cc1)CCC1O[C@@H](C)[C@H](C)O1</smiles>

(4R,5R)-2-((E)-6-(4-chlorophenyl)-3-methylhex-3-en-1-yl)-4,5-dimethyl-1,3-dioxolane (1h' for Table 3) Yield: $88 \%$. [a $]_{\mathrm{D}}{ }^{20}=29.0^{\circ}\left(c=0.99\right.$ in $\left.\mathrm{CHCl}_{3}\right) .{ }^{1} \mathrm{H} \mathrm{NMR}\left(400 \mathrm{MHz}, \mathrm{CDCl}_{3}\right): \mathrm{d} 1.22(\mathrm{~d}, J=5.8 \mathrm{~Hz}, 3 \mathrm{H}), 1.28(\mathrm{~d}$, $J=5.8 \mathrm{~Hz}, 3 \mathrm{H}), 1.53(\mathrm{~s}, 3 \mathrm{H}), 1.68-1.73(\mathrm{~m}, 2 \mathrm{H}), 2.06-2.10(\mathrm{~m}, 2 \mathrm{H}), 2.27(\mathrm{q}, J=7.4 \mathrm{~Hz}, 2 \mathrm{H}), 2.59(\mathrm{t}, J=7.6 \mathrm{~Hz}$, 
2H), 3.57-3.63 (m, 2H), $5.00(\mathrm{t}, J=4.7 \mathrm{~Hz}, 1 \mathrm{H}), 5.17(\mathrm{t}, J=7.1 \mathrm{~Hz}, 1 \mathrm{H}), 7.09(\mathrm{~d}, J=8.4 \mathrm{~Hz}, 2 \mathrm{H}), 7.22(\mathrm{~d}, J=$ $8.4 \mathrm{~Hz}, 2 \mathrm{H}) ;{ }^{13} \mathrm{C}$ NMR $\left(100 \mathrm{MHz}, \mathrm{CDCl}_{3}\right): \mathrm{d} 16.06,16.97,17.30,29.71,33.13,33.65,35.34,78.11,79.75$, 102.97, 123.20, 128.26, 129.85, 131.36, 135.47, 140.69. HRMS (ESI ${ }^{+}$) exact mass calcd for $\mathrm{C}_{18} \mathrm{H}_{26} \mathrm{ClO}_{2}$ $[\mathrm{M}+\mathrm{H}]^{+}$requires $\mathrm{m} / \mathrm{z} 309.1621$, found $\mathrm{m} / \mathrm{z} 309.1630$.<smiles>COc1ccc(CCC=C(C)CCC2O[C@@H](C)[C@@H](C)O2)cc1</smiles>

(4R,5R)-2-((E)-6-(4-methoxyphenyl)-3-methylhex-3-en-1-yl)-4,5-dimethyl-1,3-dioxolane (1g' for Table 3)

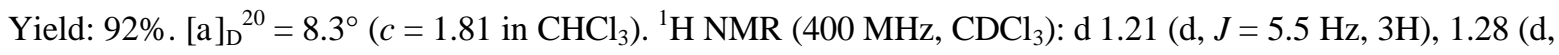
$J=5.5 \mathrm{~Hz}, 3 \mathrm{H}), 1.54$ (s, 3H), 1.69-1.74 (m, 2H), 2.06-2.10 (m, 2H), $2.26(\mathrm{q}, J=7.4 \mathrm{~Hz}, 2 \mathrm{H}), 2.56(\mathrm{t}, J=7.7 \mathrm{~Hz}$, $2 \mathrm{H}), 3.57-3.61(\mathrm{~m}, 2 \mathrm{H}), 3.76(\mathrm{~s}, 3 \mathrm{H}), 5.01(\mathrm{t}, J=4.7 \mathrm{~Hz}, 1 \mathrm{H}), 5.20(\mathrm{t}, J=6.9 \mathrm{~Hz}, 1 \mathrm{H}), 6.80(\mathrm{~d}, J=8.5 \mathrm{~Hz}, 2 \mathrm{H})$, $7.08(\mathrm{~d}, J=8.5 \mathrm{~Hz}, 2 \mathrm{H}) ;{ }^{13} \mathrm{C}$ NMR (100 MHz, $\left.\mathrm{CDCl}_{3}\right)$ : d 16.06, 16.99, 17.32, 30.17, 33.18, 33.71, 35.13, 55.19, 78.10, 79.74, 103.03, 113.63, 123.75, 129.34, 134.40, 134.99, 157.69. HRMS (ESI ${ }^{+}$) exact mass calcd for $\mathrm{C}_{19} \mathrm{H}_{29} \mathrm{O}_{3}[\mathrm{M}+\mathrm{H}]^{+}$requires $\mathrm{m} / \mathrm{z}$ 305.2117, found $\mathrm{m} / \mathrm{z} 305.2122$.<smiles>CC(=CCCCOBr)CCC1OC(C)[C@@H](C)O1</smiles>

(4R,5R)-2-((E)-7-(benzyloxy)-3-methylhept-3-en-1-yl)-4,5-dimethyl-1,3-dioxolane (1e' for Table 3)

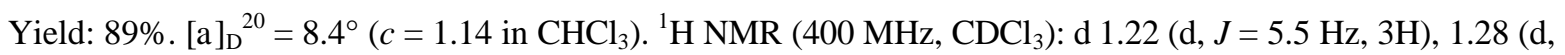
$J=5.5 \mathrm{~Hz}, 3 \mathrm{H}), 1.60(\mathrm{~s}, 3 \mathrm{H}), 1.60-1.75(\mathrm{~m}, 4 \mathrm{H}), 2.05-2.11(\mathrm{~m}, 4 \mathrm{H}), 3.45(\mathrm{t}, J=6.5 \mathrm{~Hz}, 2 \mathrm{H}), 3.57-3.60(\mathrm{~m}, 2 \mathrm{H})$, $4.48(\mathrm{~s}, 2 \mathrm{H}), 5.03(\mathrm{t}, J=4.7 \mathrm{~Hz}, 1 \mathrm{H}), 5.16(\mathrm{t}, J=6.9 \mathrm{~Hz}, 1 \mathrm{H}), 7.25-7.34(\mathrm{~m}, 5 \mathrm{H}) ;{ }^{13} \mathrm{C} \mathrm{NMR}\left(100 \mathrm{MHz}, \mathrm{CDCl}_{3}\right)$ : d 16.07, 16.99, 17.31, 24.45, 29.80, 33.24, 33.70, 69.86, 72.90, 78.10, 79.74, 103.04, 123.91, 127.47, 127.63, 128.35, 134.90, 138.68. HRMS (ESI ${ }^{+}$) exact mass calcd for $\mathrm{C}_{20} \mathrm{H}_{31} \mathrm{O}_{3}[\mathrm{M}+\mathrm{H}]^{+}$requires $\mathrm{m} / \mathrm{z} 319.2273$, found $\mathrm{m} / \mathrm{z}$ 319.2269 .<smiles>CC(=CCCc1ccccc1)CCC=O</smiles>

\section{(E)-4-methyl-7-phenylhept-4-enal (4a for Table 4)}

${ }^{1} \mathrm{H}$ NMR (400 MHz, CDCl $)$ : d 1.55 (s, 3H), 2.27-2.32 (m, 4H), 2.49 (t, $\left.J=7.4 \mathrm{~Hz}, 2 \mathrm{H}\right), 2.63$ (t, $J=7.7 \mathrm{~Hz}, 2 \mathrm{H}$ ), $5.21(\mathrm{t}, J=7.0 \mathrm{~Hz}, 1 \mathrm{H}), 7.16-7.19(\mathrm{~m}, 3 \mathrm{H}), 7.25-7.29(\mathrm{~m}, 2 \mathrm{H}), 9.73(\mathrm{~s}, 1 \mathrm{H}) ;{ }^{13} \mathrm{C} \mathrm{NMR}\left(100 \mathrm{MHz}, \mathrm{CDCl}_{3}\right): \mathrm{d}$ 16.05, 29.88, 31.83, 35.91, 42.12, 124.69, 125.77, 128.26, 128.48, 133.79, 142.08, 202.65. HRMS (ESI ${ }^{+}$) exact mass calcd for $\mathrm{C}_{14} \mathrm{H}_{19} \mathrm{O}[\mathrm{M}+\mathrm{H}]^{+}$requires $\mathrm{m} / \mathrm{z} 203.1436$, found $\mathrm{m} / \mathrm{z} 203.1434$.<smiles>CC/C=C(\C)CCC=O</smiles>

\section{(E)-4-methylhept-4-enal (4b for Table 4)}

${ }^{1} \mathrm{H}$ NMR (400 MHz, $\mathrm{CDCl}_{3}$ ): d 0.93 (t, $\left.J=7.5 \mathrm{~Hz}, 3 \mathrm{H}\right), 1.61$ (s, 3H), 1.99 (p, $J=7.2 \mathrm{~Hz}, 2 \mathrm{H}$ ), 2.32 (t $J=7.5 \mathrm{~Hz}$, $2 \mathrm{H}), 2.52(\mathrm{t}, J=7.5 \mathrm{~Hz}, 2 \mathrm{H}), 5.16(\mathrm{t}, J=6.8 \mathrm{~Hz}, 1 \mathrm{H}), 9.76(\mathrm{~s}, 1 \mathrm{H}) ;{ }^{13} \mathrm{C}$ NMR $\left(100 \mathrm{MHz}, \mathrm{CDCl}_{3}\right): \mathrm{d} 14.21,15.91$, 
21.15, 31.78, 42.15, 127.51, 132.25, 202.77. HRMS (ESI ${ }^{+}$) exact mass calcd for $\mathrm{C}_{8} \mathrm{H}_{15} \mathrm{O}[\mathrm{M}+\mathrm{H}]^{+}$requires $\mathrm{m} / \mathrm{z}$ 127.1123, found $m / 2.127 .1126$.<smiles>CCC/C=C(\C)CCC(=O)F</smiles>

\section{(E)-4-methyloct-4-enal (4c for Table 4)}

${ }^{1} \mathrm{H}$ NMR $\left(400 \mathrm{MHz}, \mathrm{CDCl}_{3}\right): \mathrm{d} 0.88(\mathrm{t}, J=7.4 \mathrm{~Hz}, 3 \mathrm{H}), 1.32-1.37(\mathrm{~m}, 2 \mathrm{H}), 1.62(\mathrm{~s}, 3 \mathrm{H}), 1.96(\mathrm{q}, J=7.2 \mathrm{~Hz}$, 2H), $2.33(\mathrm{t}, J=7.5 \mathrm{~Hz}, 2 \mathrm{H}), 2.52(\mathrm{t}, J=7.4 \mathrm{~Hz}, 2 \mathrm{H}), 5.17(\mathrm{t}, J=7.0 \mathrm{~Hz}, 1 \mathrm{H}), 9.76(\mathrm{~s}, 1 \mathrm{H}) ;{ }^{13} \mathrm{C} \mathrm{NMR}(100 \mathrm{MHz}$, $\mathrm{CDCl}_{3}$ ): d 13.76, 16.07, 22.82, 29.96, 31.90, 42.19, 125.73, 132.93, 202.78. HRMS (ESI ${ }^{+}$) exact mass calcd for $\mathrm{C}_{9} \mathrm{H}_{17} \mathrm{O}[\mathrm{M}+\mathrm{H}]^{+}$requires $m / z$ 141.1279, found $m / z$ 141.1278.<smiles>COc1ccc(CC/C=C(\C)CCC=O)cc1</smiles>

\section{(E)-7-(4-methoxyphenyl)-4-methylhept-4-enal (4d for Table 4)}

${ }^{1} \mathrm{H}$ NMR $\left(400 \mathrm{MHz}, \mathrm{CDCl}_{3}\right.$ ): d 1.55 (s, 3H), 2.26-2.32 (m, 4H), 2.49 (t, $\left.J=7.1 \mathrm{~Hz}, 2 \mathrm{H}\right), 2.57$ (t, $\left.J=7.7 \mathrm{~Hz}, 2 \mathrm{H}\right)$, $3.78(\mathrm{~s}, 3 \mathrm{H}), 5.19(\mathrm{t}, J=7.0 \mathrm{~Hz}, 1 \mathrm{H}), 6.82(\mathrm{~d}, J=8.4 \mathrm{~Hz}, 2 \mathrm{H}), 7.08(\mathrm{~d}, J=8.4 \mathrm{~Hz}, 2 \mathrm{H}), 9.73(\mathrm{~s}, 1 \mathrm{H}) ;{ }^{13} \mathrm{C} \mathrm{NMR}$ $\left(100 \mathrm{MHz}, \mathrm{CDCl}_{3}\right)$ : d 16.06, 30.11, 31.83, 34.98, 42.12, 55.26, 113.66, 124.77, 129.34, 133.68, 134.19, 157.75, 202.69. $\mathrm{HRMS}\left(\mathrm{ESI}^{+}\right.$) exact mass calcd for $\mathrm{C}_{15} \mathrm{H}_{20} \mathrm{O}_{2} \mathrm{Na}[\mathrm{M}+\mathrm{Na}]^{+}$requires $m / z, 255.1361$, found $m / z$ 255.1357.<smiles>C=C(C)CCC=O</smiles>

\section{4-Methylpent-4-enal (4e for Table 4)}

${ }^{1} \mathrm{H}$ NMR (400 MHz, CDCl $)$ ) d 1.75 (s, 3H), $2.35(\mathrm{t}, J=7.4 \mathrm{~Hz}, 2 \mathrm{H}), 2.58\left(\mathrm{dt}, J_{l}=1.3 \mathrm{~Hz}, J_{2}=7.3 \mathrm{~Hz}, 2 \mathrm{H}\right.$ ), 4.69 (s, 1H), 4.77 (s, 1H), 9.78 (t, $J=1.7 \mathrm{~Hz}, 1 \mathrm{H}) ;{ }^{13} \mathrm{C} \mathrm{NMR}\left(100 \mathrm{MHz}, \mathrm{CDCl}_{3}\right)$ : d 22.61, 29.82, 41.76, 110.68, 143.75, 202.23. HRMS (ESI ${ }^{+}$) exact mass calcd for $\mathrm{C}_{6} \mathrm{H}_{11} \mathrm{O}[\mathrm{M}+\mathrm{H}]^{+}$requires $\mathrm{m} / \mathrm{z}$ 99.0810, found $\mathrm{m} / z$ 99.0811.<smiles>O=CCCC1=CCCCCC1</smiles>

\section{3-(Cyclohept-1-en-1-yl)propanal (4f for Table 4)}

${ }^{1} \mathrm{H}$ NMR (400 MHz, $\mathrm{CDCl}_{3}$ ): d 1.41-1.50 (m, 4H), 1.70-1.76 (m, 2H), 2.04-2.11 (m, 4H), $2.32(\mathrm{t}, J=7.4 \mathrm{~Hz} 2 \mathrm{H})$, $2.49\left(\mathrm{dt}, J_{l}=1.9 \mathrm{~Hz}, J_{2}=7.4 \mathrm{~Hz}, 2 \mathrm{H}\right), 5.57(\mathrm{t}, J=6.4 \mathrm{~Hz}, 1 \mathrm{H}), 9.75(\mathrm{t}, J=1.9 \mathrm{~Hz}, 1 \mathrm{H}) ;{ }^{13} \mathrm{C}$ NMR $(100 \mathrm{MHz}$, $\mathrm{CDCl}_{3}$ ): $\mathrm{d} 26.66,27.14,28.20,32.43,32.53,32.83,42.17,127.01,142.49,202.90$. HRMS $\left(\mathrm{ESI}^{+}\right.$) exact mass calcd for $\mathrm{C}_{10} \mathrm{H}_{17} \mathrm{O}[\mathrm{M}+\mathrm{H}]^{+}$requires $m / z, 153.1279$, found $m / z$ 153.1277.

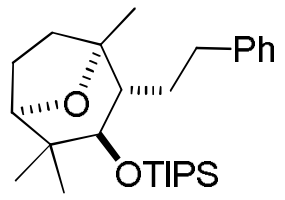

The enantiomeric excess was determined by chiral HPLC (Chiralpak OD-H, $0.1 \% i$-PrOH/hexanes, flow rate $1.0 \mathrm{~mL} / \mathrm{min}, ?=220 \mathrm{~nm}$ ); $t_{\mathrm{r}}=7.37 \mathrm{~min}$ (major) and $11.62 \mathrm{~min}$ (minor) $\min .97 \%$ ee. Yield: $88 \%$. $[\mathrm{a}]_{\mathrm{D}}{ }^{20}=27.9^{\circ}$ $\left(c=1.52\right.$ in $\left.\mathrm{CHCl}_{3}\right)$. 


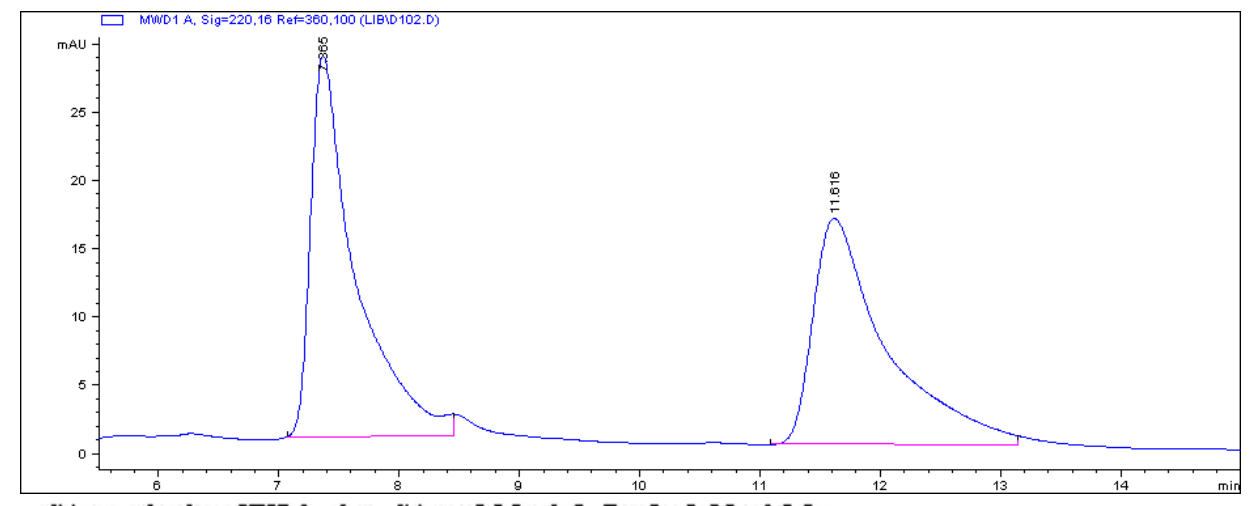

Signal 1: MJDl A, Sig=220,16 Ref $=360,100$

\begin{tabular}{|c|c|c|c|c|c|c|}
\hline $\begin{array}{c}\text { Peak } \\
\#\end{array}$ & $\begin{array}{c}\text { RetTime } \\
\text { [min] }\end{array}$ & Type & $\begin{array}{l}\text { Width } \\
\text { [ min] }\end{array}$ & $\begin{array}{c}\text { Area } \\
{\left[\mathrm{m} h U^{*} s\right]}\end{array}$ & $\begin{array}{l}\text { Height } \\
\text { [mÄU] }\end{array}$ & $\begin{array}{c}\text { Area } \\
\stackrel{4}{*}\end{array}$ \\
\hline$=-$ & ------ & & ------- & ---------1 & ------ & ---1 \\
\hline 1 & 7.365 & $\mathrm{BB}$ & 0.3637 & 726.02765 & 27.89085 & 160 \\
\hline 2 & 11.616 & $\mathrm{BB}$ & 0.5976 & 699.90558 & 16.54170 & 49.0840 \\
\hline
\end{tabular}

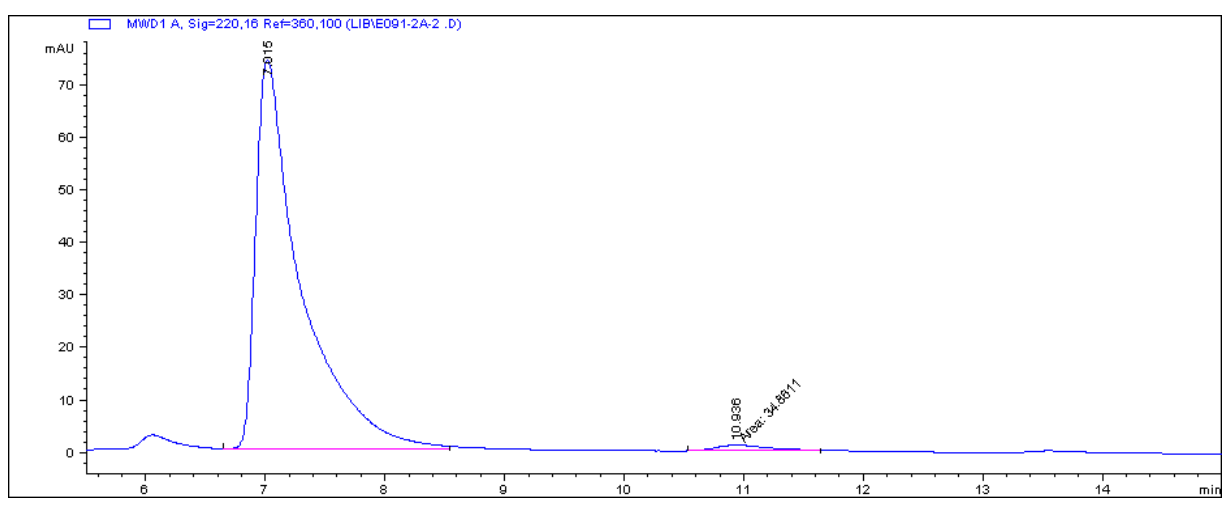

Signal 1: MTDl À, Sig=220,16 Ref $=360,100$

\begin{tabular}{|c|c|c|c|c|c|c|}
\hline $\begin{array}{c}\text { Peak } \\
\#\end{array}$ & $\begin{array}{c}\text { RetTime } \\
\text { [min] }\end{array}$ & Type & $\begin{array}{l}\text { Width } \\
\text { [min] }\end{array}$ & $\begin{array}{c}\text { Area } \\
{\left[\mathrm{m} \mathrm{H}^{*} s\right]}\end{array}$ & $\begin{array}{l}\text { Height } \\
\text { [யயAU] }\end{array}$ & $\begin{array}{c}\text { Area } \\
*\end{array}$ \\
\hline-- & ------ & & ----- & $|----------|$ & ------ & ----1 \\
\hline 1 & 7.015 & VB & 0.3672 & 1951.85181 & 74.12254 & 98.2453 \\
\hline 2 & 10.936 & MM & 0.5328 & 34.86113 & 1. 09049 & 1.7547 \\
\hline
\end{tabular}

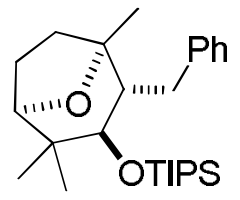

The enantiomeric excess was determined by chiral HPLC (Chiralpak AD-H, $0.1 \% i$-PrOH/hexanes, flow rate $1.0 \mathrm{~mL} / \mathrm{min}, ?=254 \mathrm{~nm}) ; t_{\mathrm{r}}=5.18 \mathrm{~min}$ (major) and $5.68 \mathrm{~min}\left(\right.$ minor) $\min .94 \%$ ee. Yield: $91 \% \cdot[\mathrm{a}]_{\mathrm{D}}{ }^{20}=-16.2^{\circ}$ $\left(c=2.60\right.$ in $\left.\mathrm{CHCl}_{3}\right)$. 


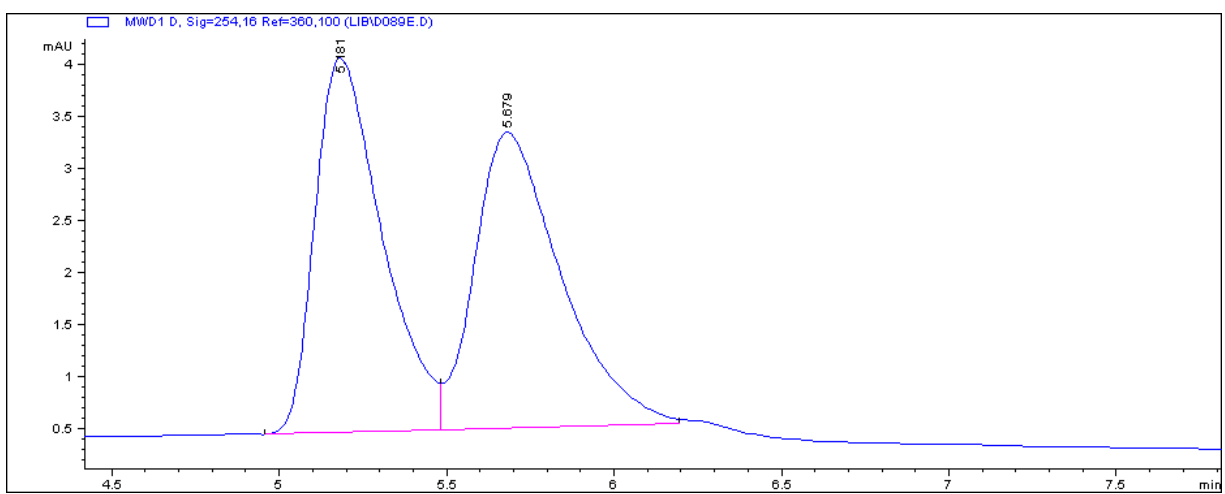

Signal 4: MWD 1 D, Sig $=254,16$ Ref $=360,100$

\begin{tabular}{|c|c|c|c|c|c|c|}
\hline $\begin{array}{c}\text { Peak } \\
\#\end{array}$ & $\begin{array}{c}\text { RetTime } \\
\text { [min] }\end{array}$ & Type & $\begin{array}{l}\text { Width } \\
\text { [min] }\end{array}$ & $\begin{array}{c}\text { Area } \\
{\left[\mathrm{m} A U^{*} s\right]}\end{array}$ & $\begin{array}{l}\text { Height } \\
\text { [ miU] ] }\end{array}$ & $\begin{array}{c}\text { Area } \\
\stackrel{4}{*}\end{array}$ \\
\hline & - & & & ------ & - ------ & -----1 \\
\hline 1 & 5.181 & $\mathrm{BV}$ & 0.2113 & 50.25304 & 3.59111 & 49.6802 \\
\hline 2 & 5.679 & VB & 0.2656 & 50.89997 & 2.83311 & 50.3198 \\
\hline
\end{tabular}
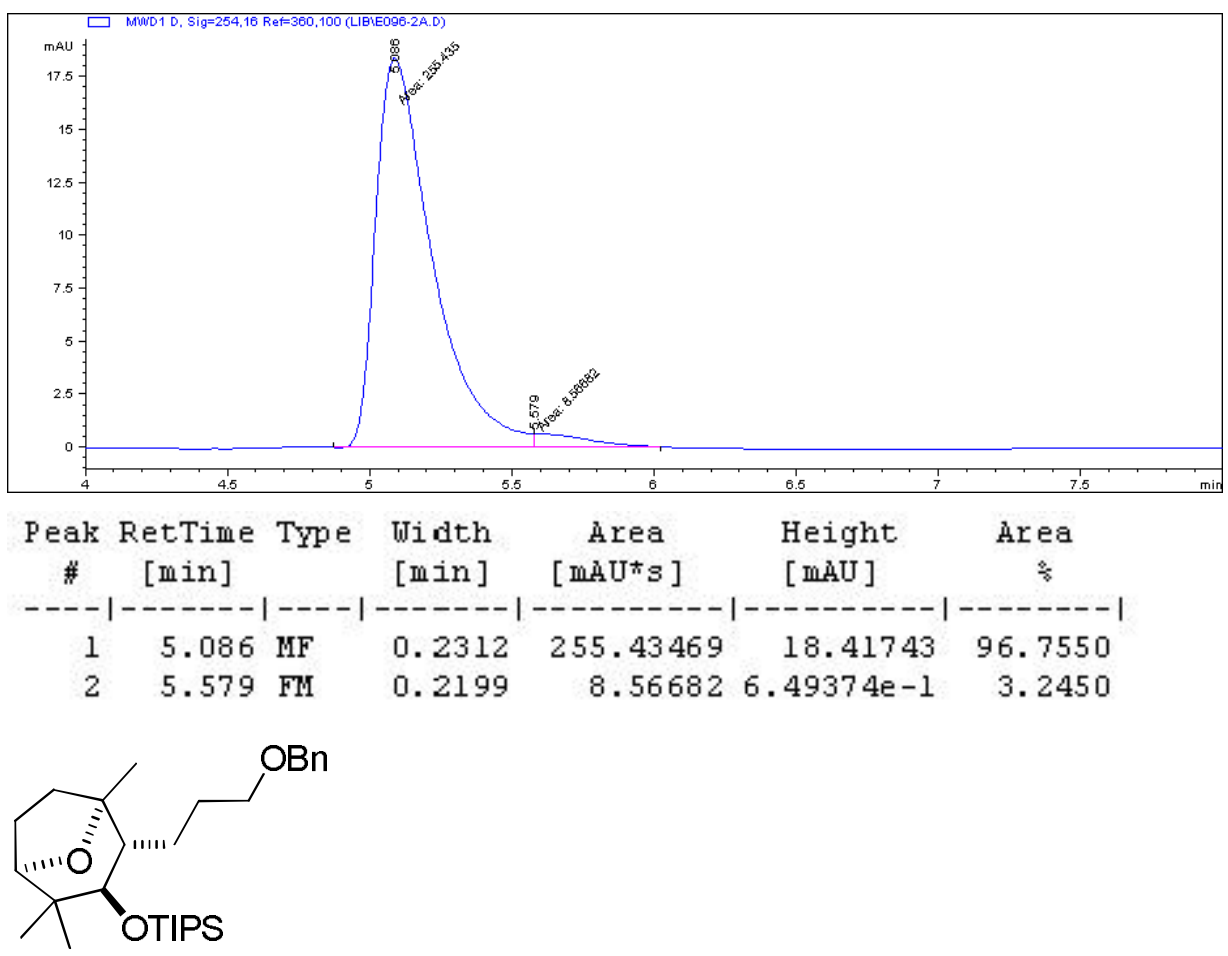

The enantiomeric excess was determined by chiral HPLC (Chiralpak OD-H, $0.5 \% i$-PrOH/hexanes, flow rate $1.0 \mathrm{~mL} / \mathrm{min}, ?=220 \mathrm{~nm}$ ); $\mathrm{t}_{\mathrm{r}}=5.24 \mathrm{~min}$ (major) and $9.13 \mathrm{~min}$ (minor) $\mathrm{min} .94 \%$ ee. Yield: $79 \%$. $[\mathrm{a}]_{\mathrm{D}}{ }^{20}=4.6^{\circ}(c$ $=1.68$ in $\mathrm{CHCl}_{3}$ ). 


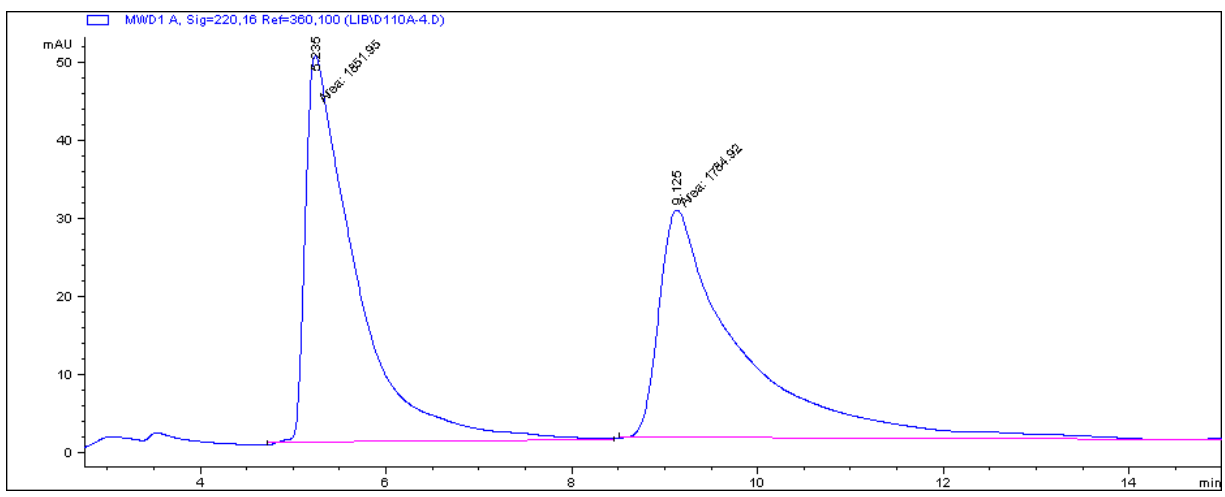

Signal 1: MWD1 A, Sig=220,16 Ref $=360,100$

\begin{tabular}{|c|c|c|c|c|c|c|}
\hline $\begin{array}{c}\text { Peak } \\
\#\end{array}$ & $\begin{array}{c}\text { Re tTime } \\
\text { [min] }\end{array}$ & Type & $\begin{array}{l}\text { Width } \\
\text { [ min] }\end{array}$ & $\begin{array}{c}\text { Area } \\
\left.\text { [min } U^{*} s\right]\end{array}$ & $\begin{array}{l}\text { Height } \\
\text { [mAU] }\end{array}$ & $\begin{array}{c}\text { Àrea } \\
\stackrel{5}{*}\end{array}$ \\
\hline - & & & - & -----0 & & ---1 \\
\hline 1 & 5.235 & MM & 0.6240 & 1851.94946 & 49.46545 & 50.9215 \\
\hline 2 & 9.125 & $M M$ & 1.0193 & 1784.92236 & 29.18522 & 49.0785 \\
\hline
\end{tabular}

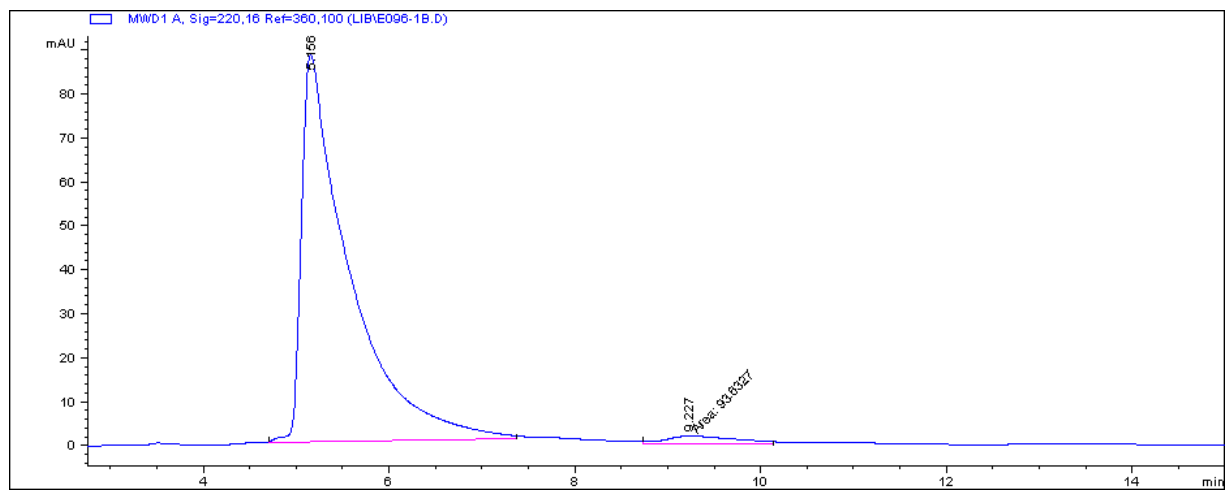

Signal 1: MUDl A, Sig=220,16 Ref $=360,100$

\begin{tabular}{|c|c|c|c|c|c|c|}
\hline $\begin{array}{c}\text { Peak } \\
\#\end{array}$ & $\begin{array}{c}\text { Re tTime } \\
\text { [min] }\end{array}$ & Type & $\begin{array}{l}\text { Uidth } \\
\text { [min] }\end{array}$ & $\begin{array}{c}\text { Area } \\
{\left[\mathrm{m} h U^{*} s\right]}\end{array}$ & $\begin{array}{l}\text { Height } \\
\text { [mAU] }\end{array}$ & $\begin{array}{c}\text { Area } \\
\stackrel{5}{*}\end{array}$ \\
\hline$=-$ & ----- & & ------- & ---------- & 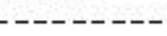 & -------1 \\
\hline 1 & 5.156 & $\mathrm{BB}$ & 0.4800 & 3172.93408 & 88.12731 & 97.1336 \\
\hline 2 & 9.227 & $M M$ & 0.8742 & 93.63274 & 1. 78508 & 2.8664 \\
\hline
\end{tabular}

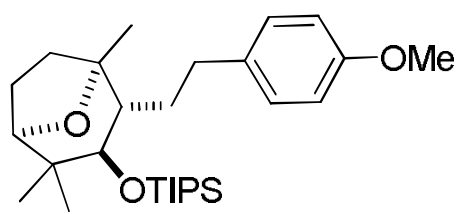

The enantiomeric excess was determined by chiral HPLC (Chiralpak AD-H, 0.5\% $i$-PrOH/hexanes, flow rate $1.0 \mathrm{~mL} / \mathrm{min}, ?=220 \mathrm{~nm}$ ); $t_{\mathrm{r}}=8.78 \mathrm{~min}$ (minor) and $9.53 \mathrm{~min}$ (major) $\min .95 \%$ ee. Yield: $74 \% .\left[\mathrm{a}_{\mathrm{D}}{ }^{20}=29.6^{\circ}\right.$ $\left(c=1.98\right.$ in $\left.\mathrm{CHCl}_{3}\right)$. 


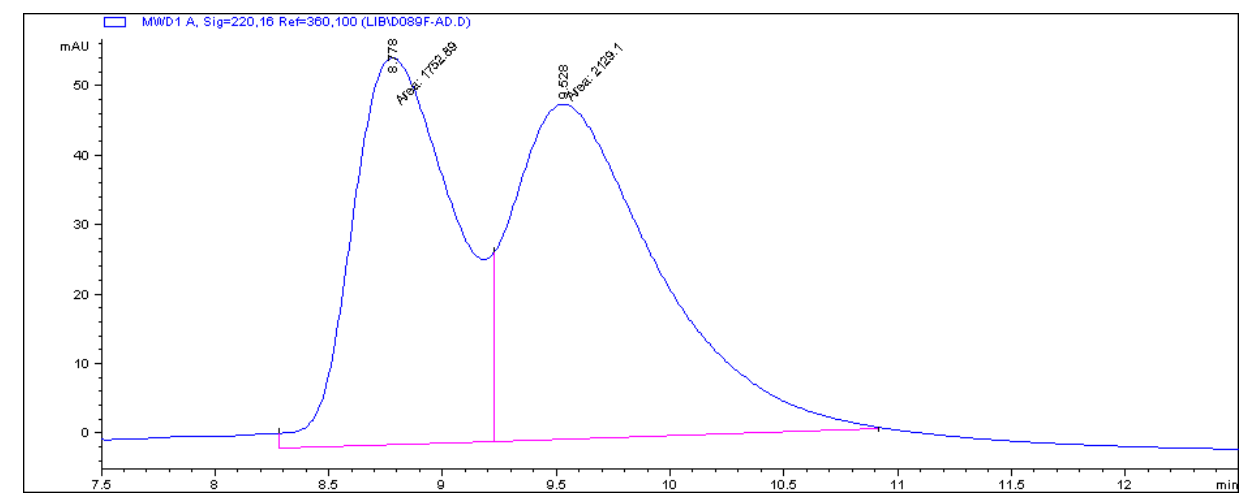

Signal 1: MJDl Aे, Sig=220,16 Ref $=360,100$

\begin{tabular}{|c|c|c|c|c|c|c|}
\hline $\begin{array}{c}\text { Peak } \\
\#\end{array}$ & $\begin{array}{l}\text { Re tTime } \\
\text { [min] }\end{array}$ & Type & $\begin{array}{l}\text { Wid th } \\
\text { [min] }\end{array}$ & $\begin{array}{c}\text { Area } \\
\text { [mAU*'s] }\end{array}$ & $\begin{array}{l}\text { Height } \\
\text { [mAU] }\end{array}$ & $\begin{array}{c}\text { Area } \\
\frac{5}{8}\end{array}$ \\
\hline-0 & ------ & & ----- & --------- & ----- & ------1 \\
\hline 1 & 8.778 & MF & 0.5228 & 1752.88940 & 55.88053 & 45.1544 \\
\hline 2 & 9.528 & FM & 0.7330 & 2129.10498 & 48.41377 & 54.8456 \\
\hline
\end{tabular}

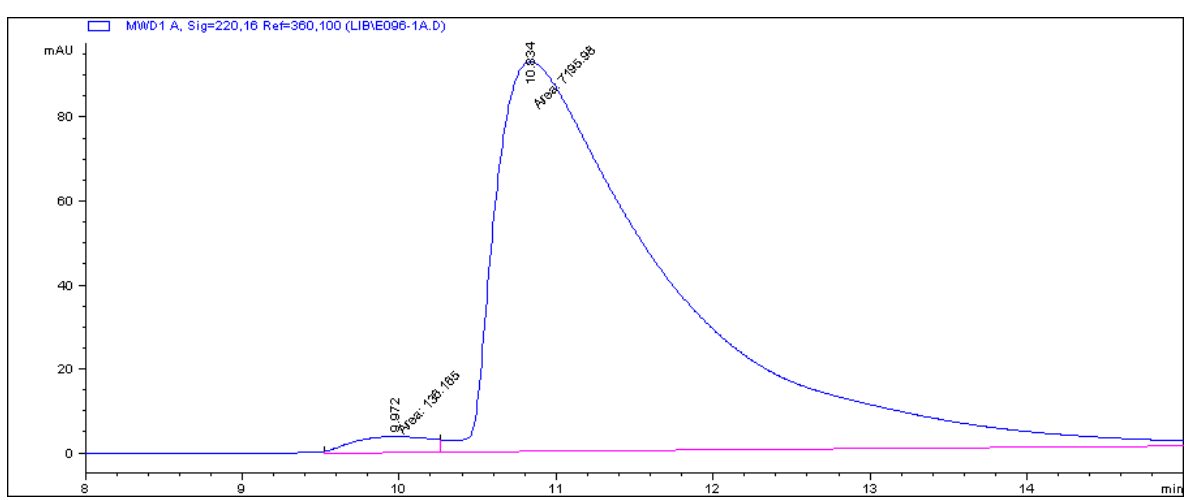

Signal 1: MUD 1 A, Sig $=220,16$ Ref $=360,100$
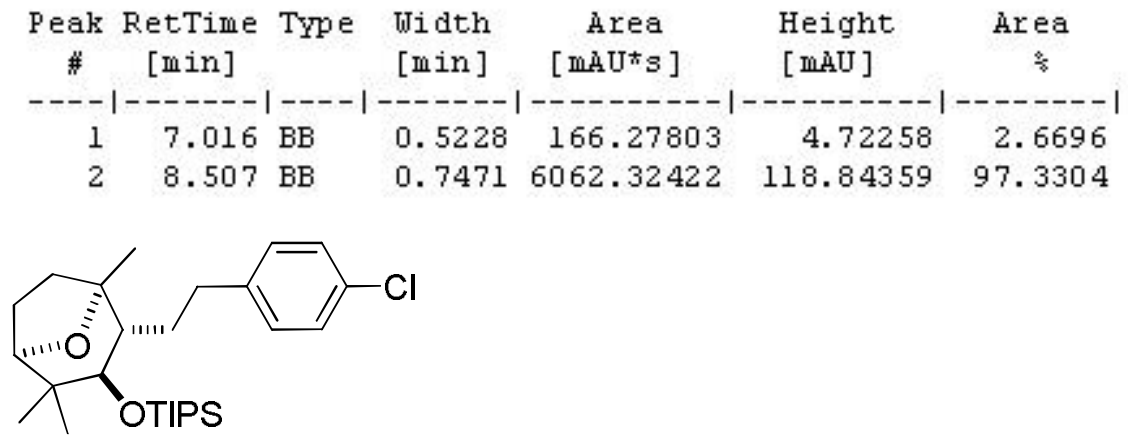

The enantiomeric excess was determined by chiral HPLC (Chiralpak AD-H + OD-H, $0.1 \% i$-PrOH/hexanes, flow rate $0.7 \mathrm{~mL} / \mathrm{min}, ?=220 \mathrm{~nm}$ ); $t_{\mathrm{r}}=17.82 \mathrm{~min}$ (minor) and $19.13 \mathrm{~min}$ (major) $\mathrm{min} .98 \%$ ee. Yield: $80 \%$. $[\mathrm{a}]_{\mathrm{D}}^{20}=32.9^{\circ}\left(c=2.93\right.$ in $\left.\mathrm{CHCl}_{3}\right)$. 


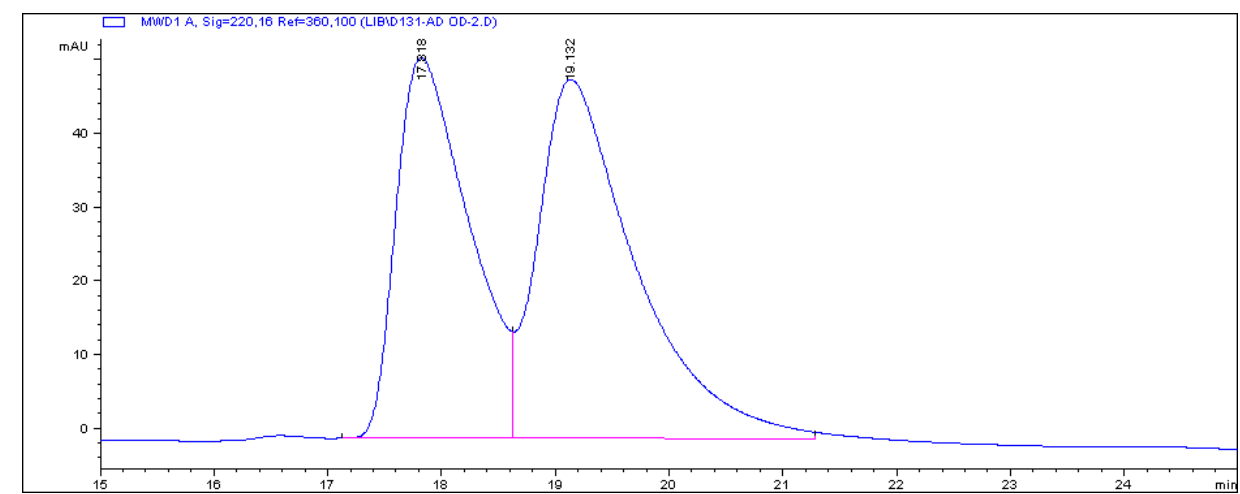

Signal 1: MJDDl A, Sig $=220,16$ Ref $=360,100$

\begin{tabular}{|c|c|c|c|c|c|c|}
\hline $\begin{array}{c}\text { Peak } \\
\#\end{array}$ & $\begin{array}{c}\text { RetTime } \\
\text { [min] }\end{array}$ & Type & $\begin{array}{l}\text { Width } \\
\text { [min] }\end{array}$ & $\begin{array}{c}\text { Area } \\
{\left[\mathrm{m} \mathrm{H}^{*} \mathrm{~s}\right]}\end{array}$ & $\begin{array}{l}\text { Height } \\
\text { [mAU] }\end{array}$ & Area \\
\hline--- & & & 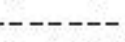 & 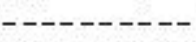 & ------ & -------1 \\
\hline 1 & 17.818 & $\mathrm{BV}$ & 0.6712 & 2316.30078 & 51.64791 & 44. 1935 \\
\hline 2 & 19.132 & VB & 0.8677 & 2924.97217 & 48.76922 & 55.8065 \\
\hline
\end{tabular}

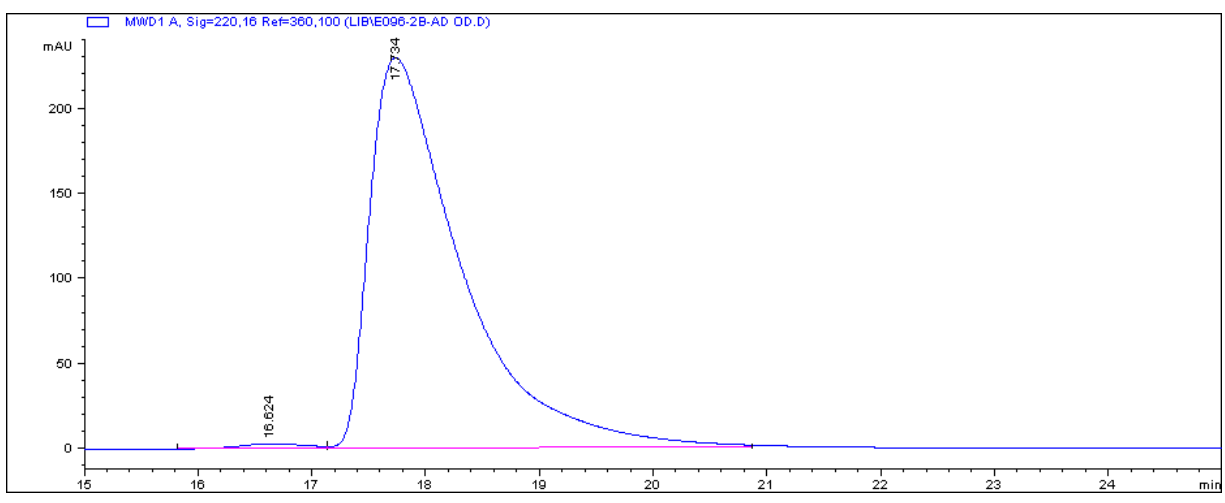

Signal 1: MJD1 म, Sig=220, 16 Ref $=360,100$

\begin{tabular}{|c|c|c|c|c|c|c|}
\hline $\begin{array}{c}\text { Peak } \\
\#\end{array}$ & $\begin{array}{c}\text { Re tTime } \\
\text { [min] }\end{array}$ & Type & $\begin{array}{l}\text { Width } \\
\text { [min] }\end{array}$ & $\begin{array}{c}\text { Area } \\
{\left[\mathrm{m} n U^{*} s\right]}\end{array}$ & $\begin{array}{l}\text { Height } \\
\text { [mAU] }\end{array}$ & $\begin{array}{c}\text { Area } \\
\frac{\Delta}{*}\end{array}$ \\
\hline & & & & --------- & ------ & ------- \\
\hline 1 & 16.624 & $\mathrm{BV}$ & 0.6624 & 130.37111 & 3.07639 & 0.9985 \\
\hline 2 & 17.734 & VB & 0.8217 & $1.29265 \mathrm{e} 4$ & 229.30748 & 99.0015 \\
\hline
\end{tabular}




\section{Spectrum}

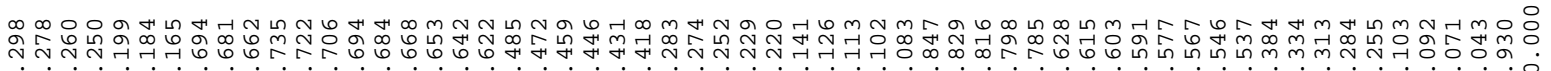

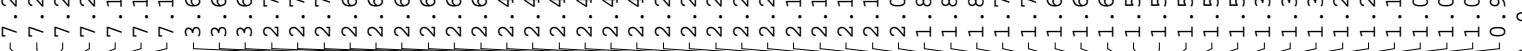

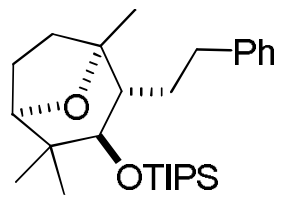

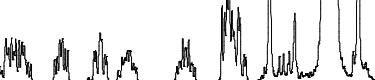

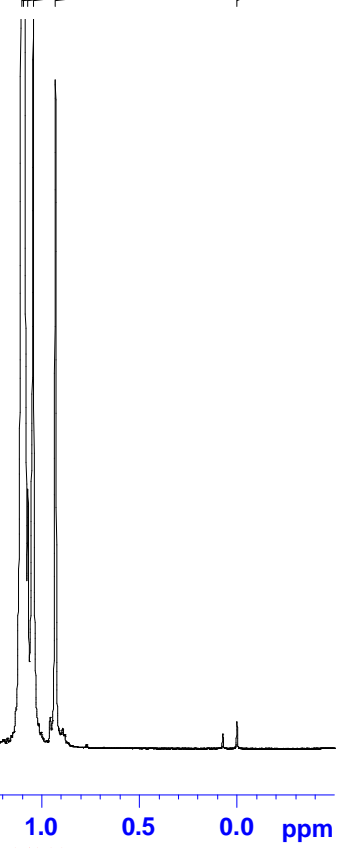
$|\bar{\Gamma}| \stackrel{\text { N. }}{\operatorname{s}} \mid$
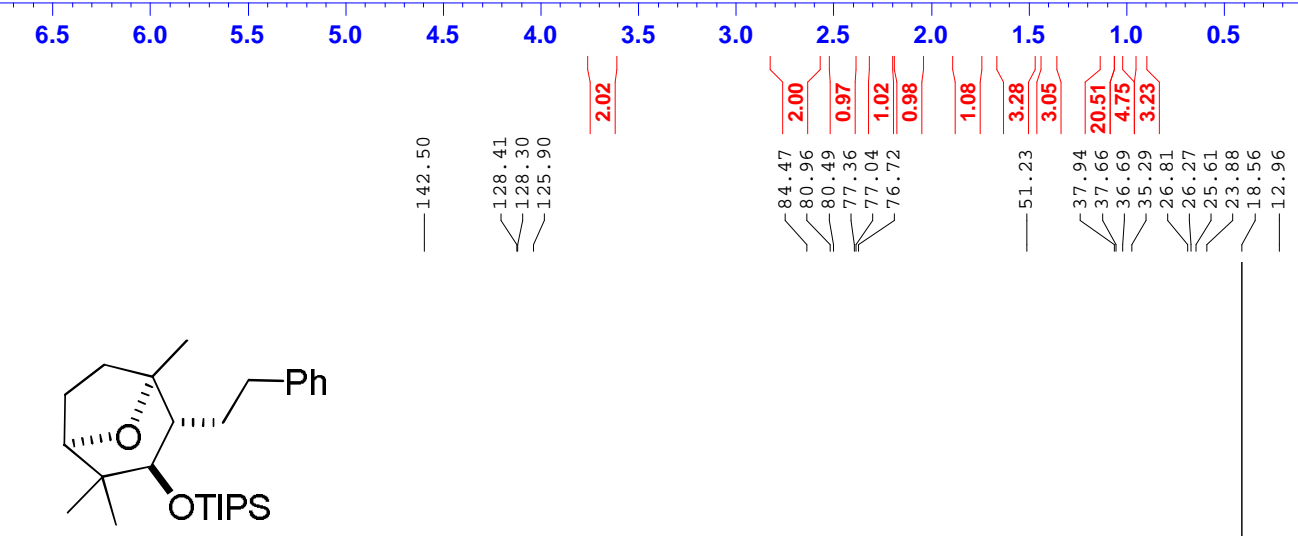


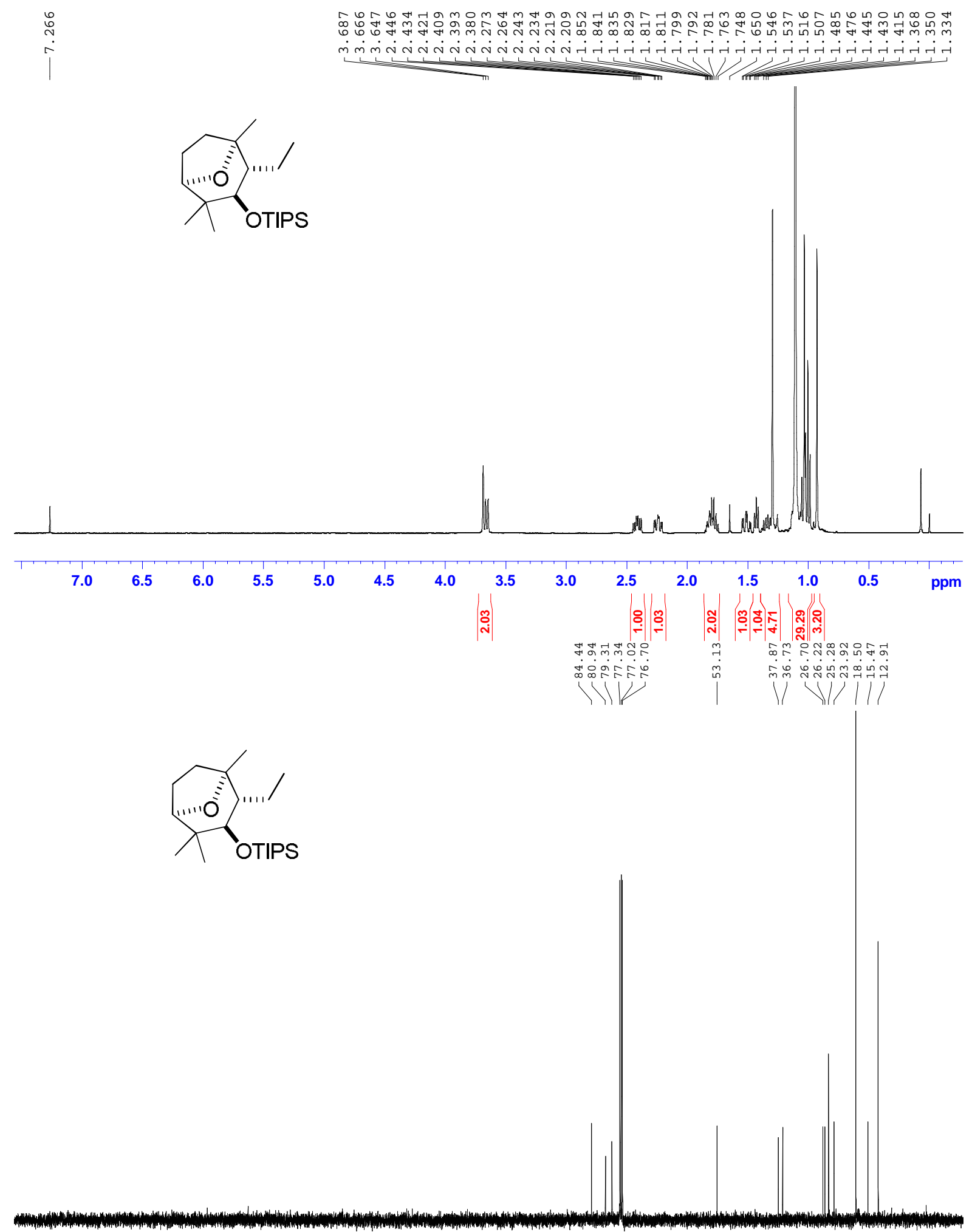

$\begin{array}{lllllllllllllllllllllll}220 & 210 & 200 & 190 & 180 & 170 & 160 & 150 & 140 & 130 & 120 & 110 & 100 & 90 & 80 & 70 & 60 & 50 & 40 & 30 & 20 & 10 & \text { ppm }\end{array}$ 

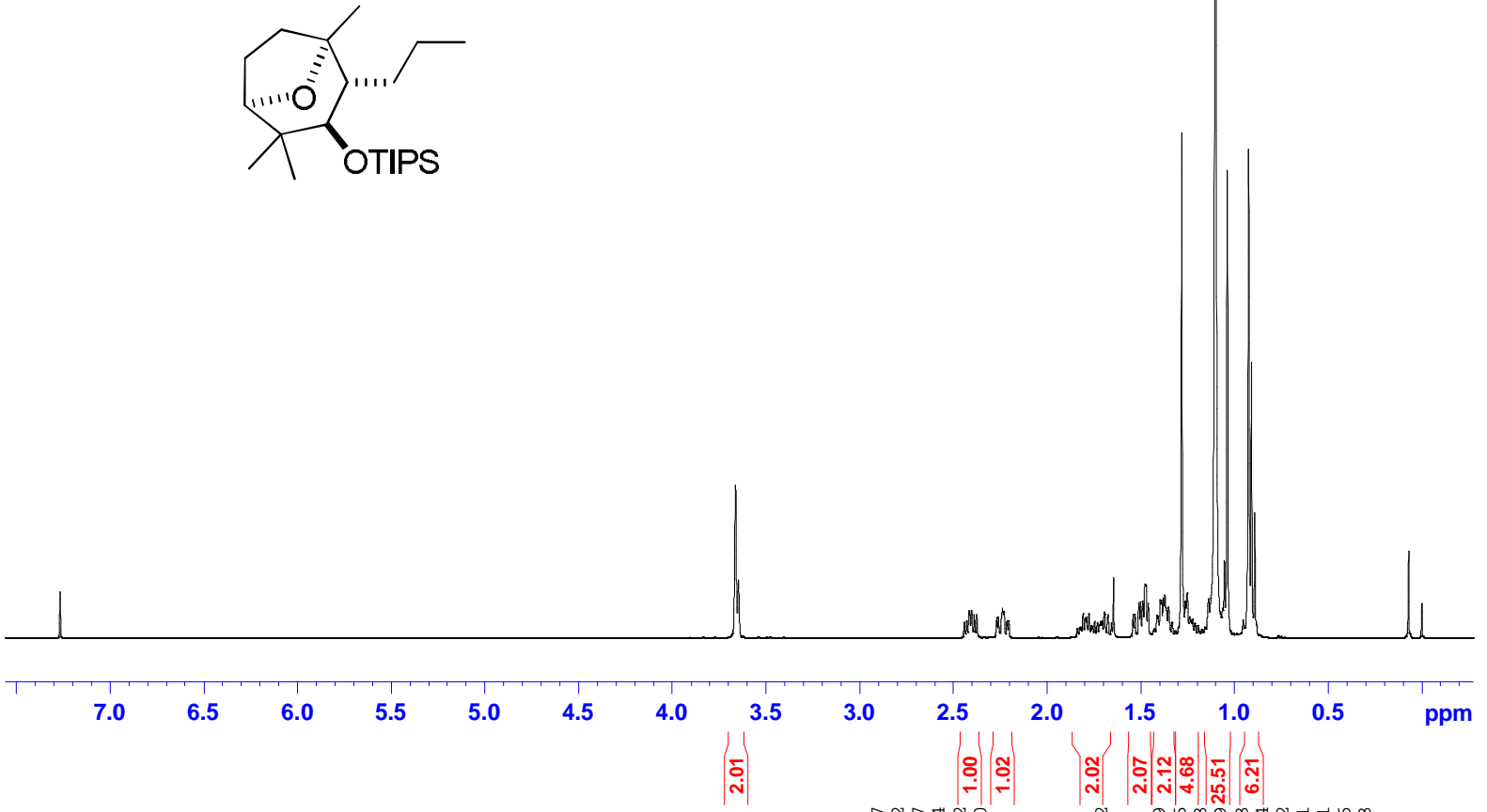

2.0

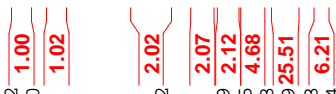

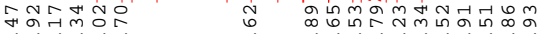

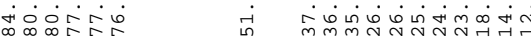

$\left.\right|_{1} ^{\infty}$

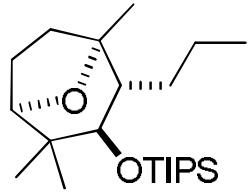

$\begin{array}{lllllllllllllllllllllll}220 & 210 & 200 & 190 & 180 & 170 & 160 & 150 & 140 & 130 & 120 & 110 & 100 & 90 & 80 & 70 & 60 & 50 & 40 & 30 & 20 & 10 & \text { ppm }\end{array}$ 

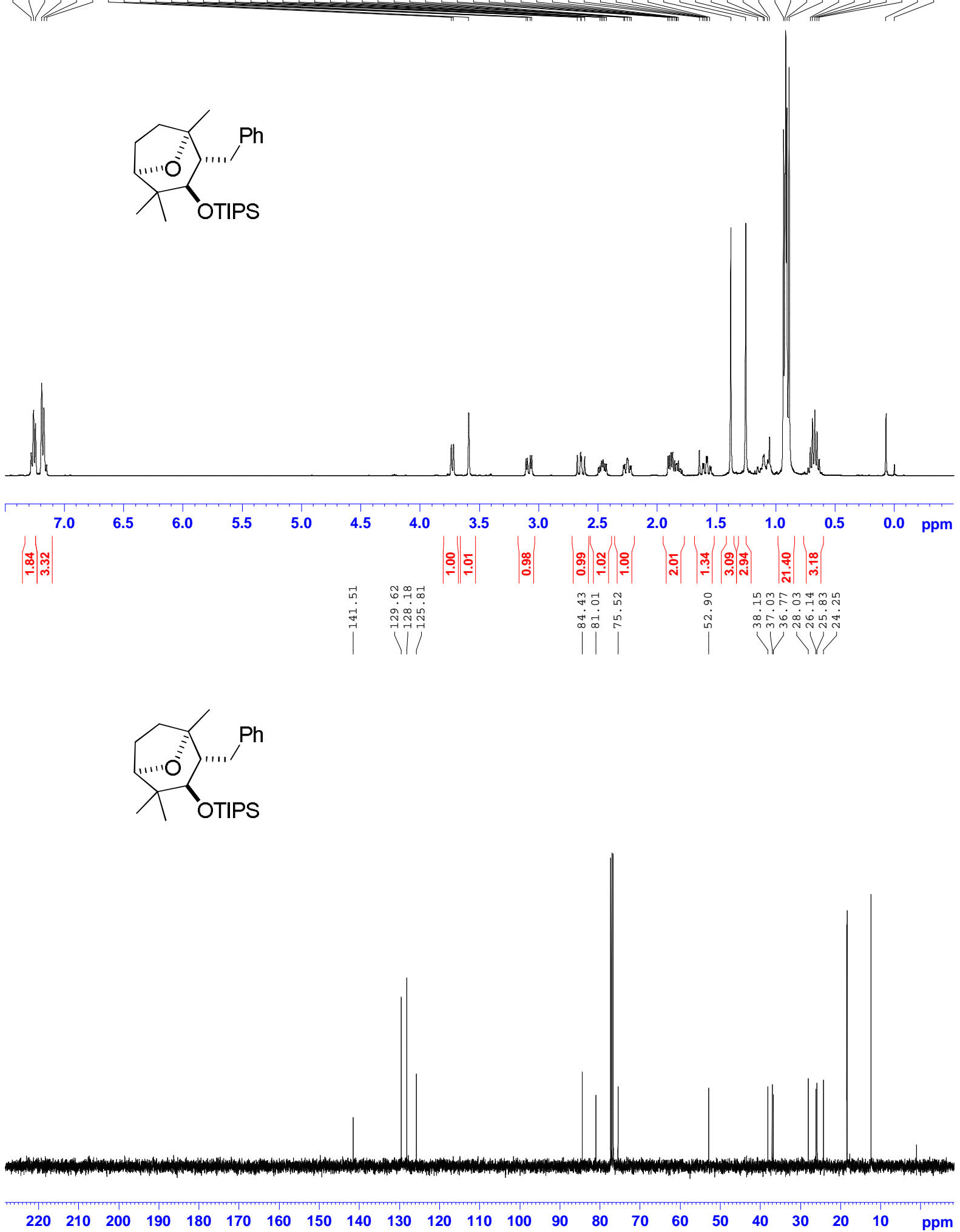

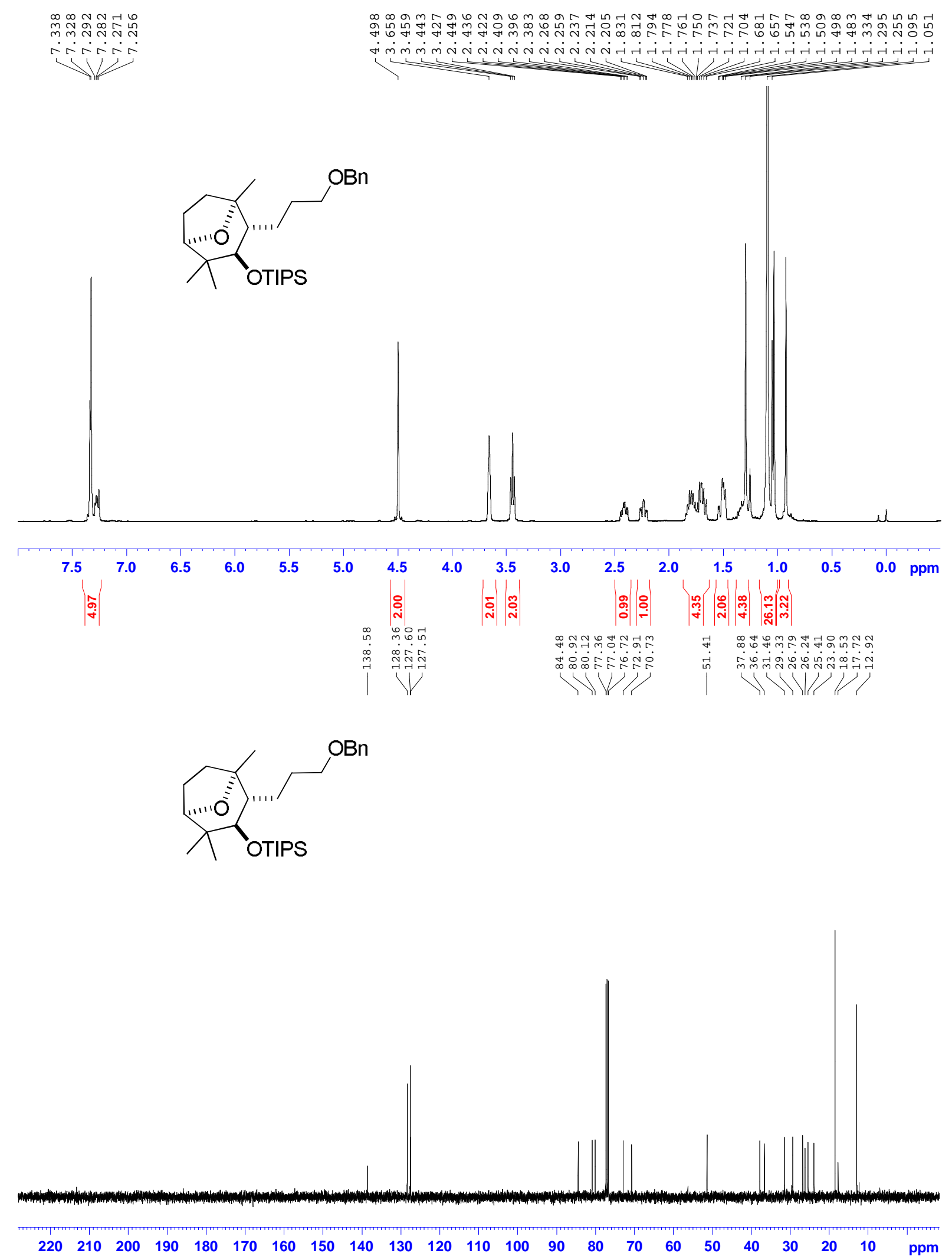

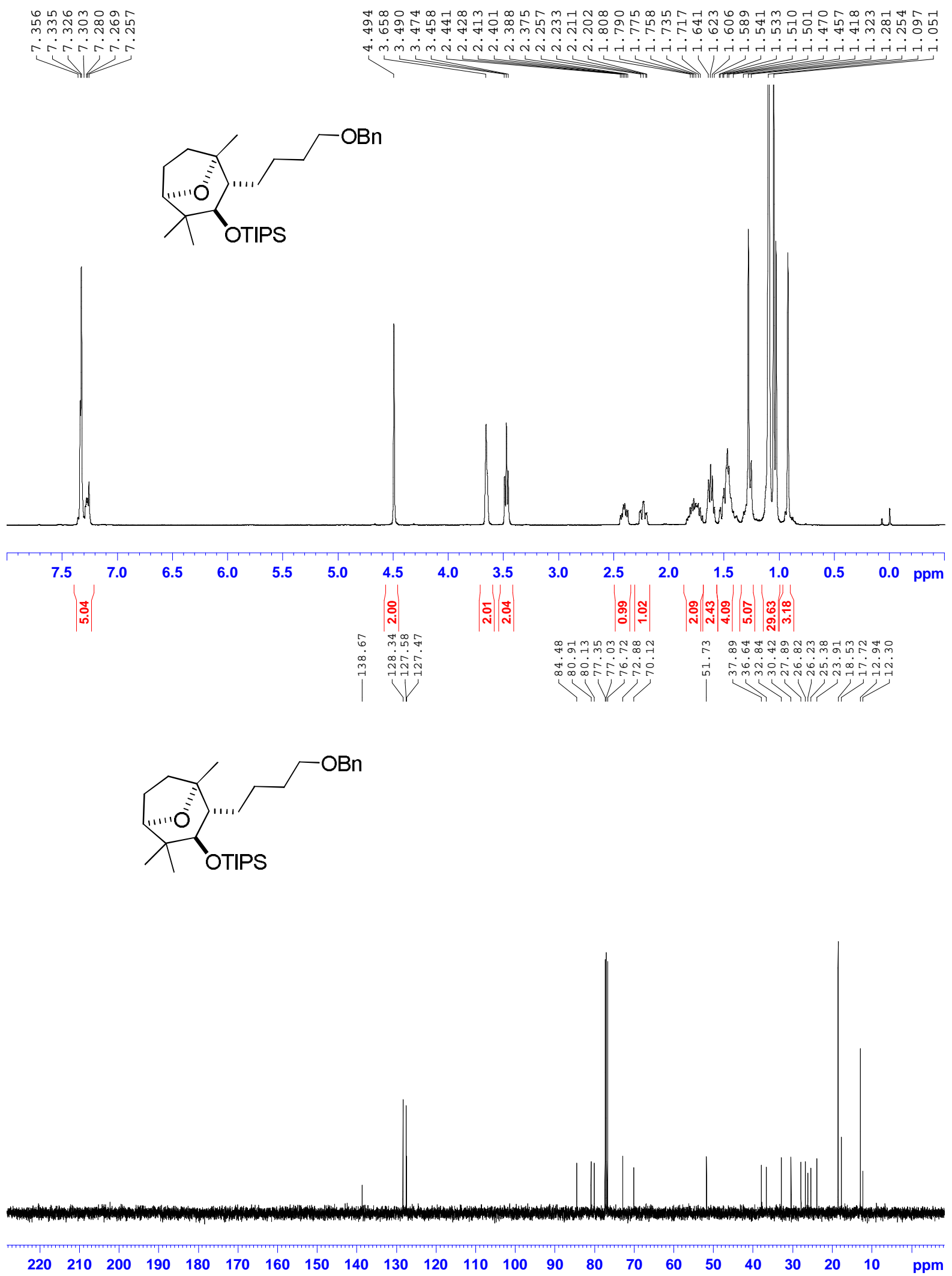

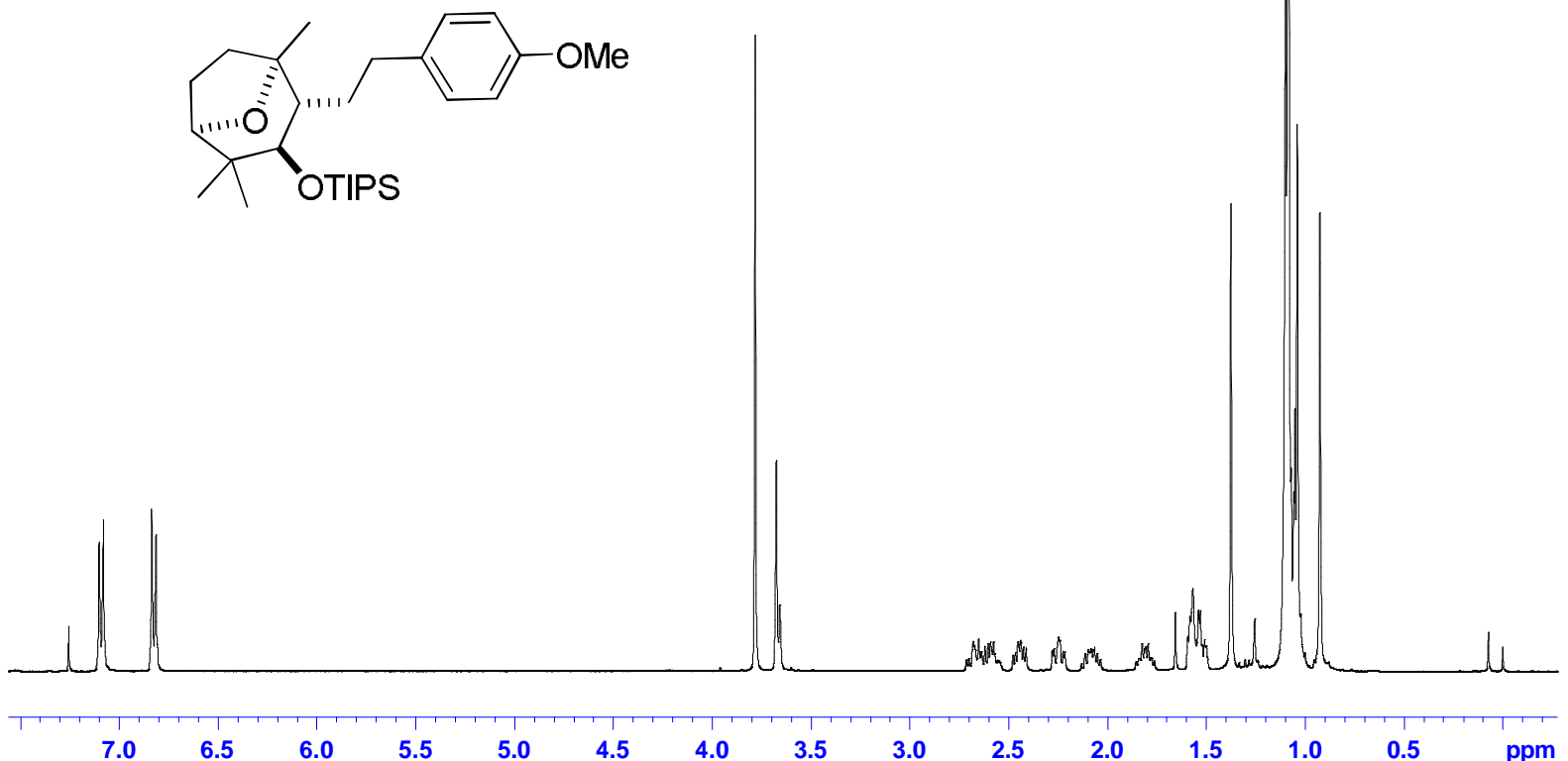

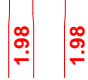

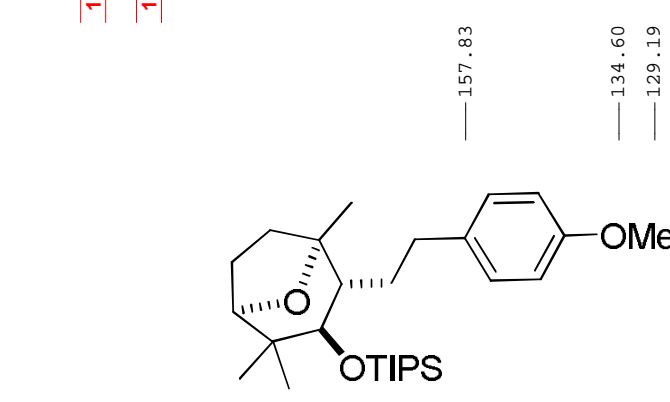

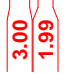

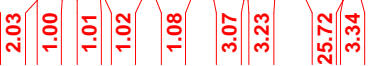

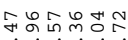

कis

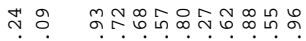

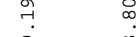

UV

i.

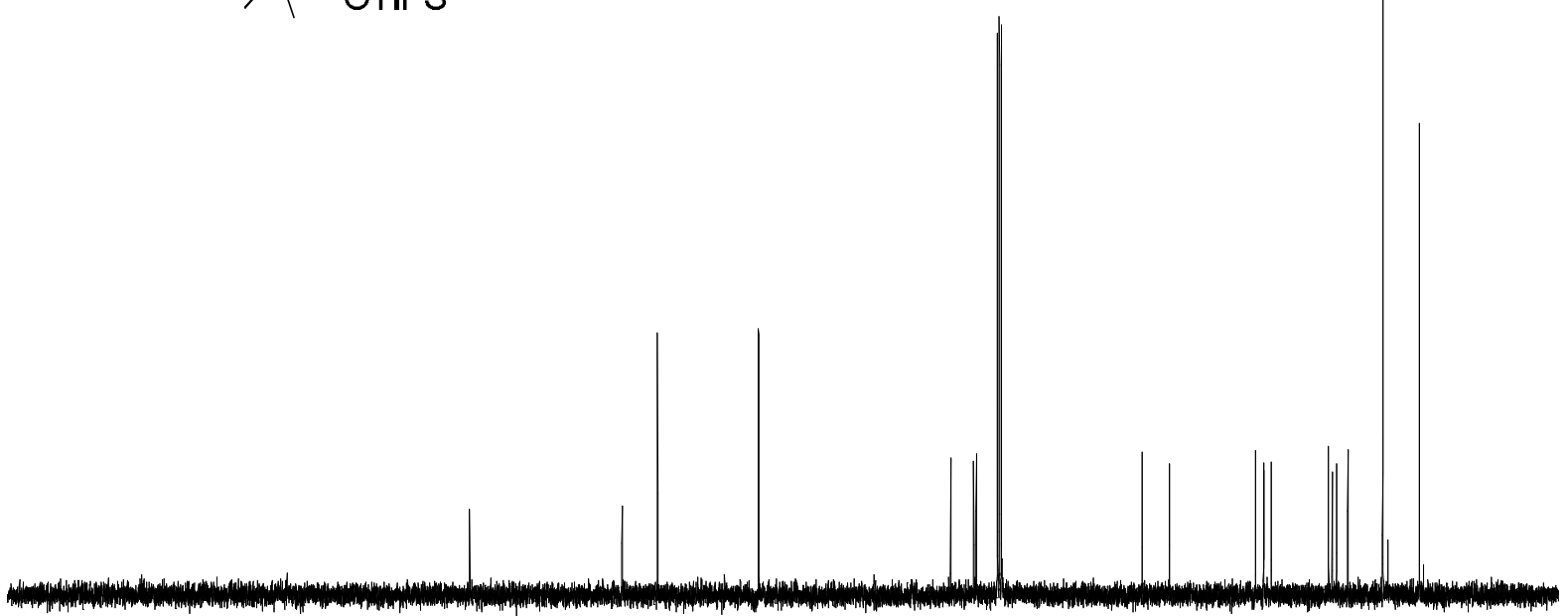

$\begin{array}{lllllllllllllllllllllll}220 & 210 & 200 & 190 & 180 & 170 & 160 & 150 & 140 & 130 & 120 & 110 & 100 & 90 & 80 & 70 & 60 & 50 & 40 & 30 & 20 & 10 & \mathrm{ppm}\end{array}$ 


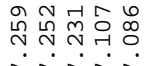

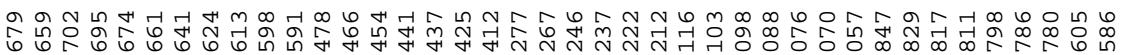

$\sin 40$

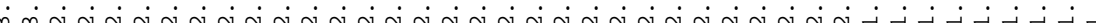

Vy

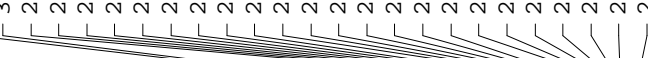

1)
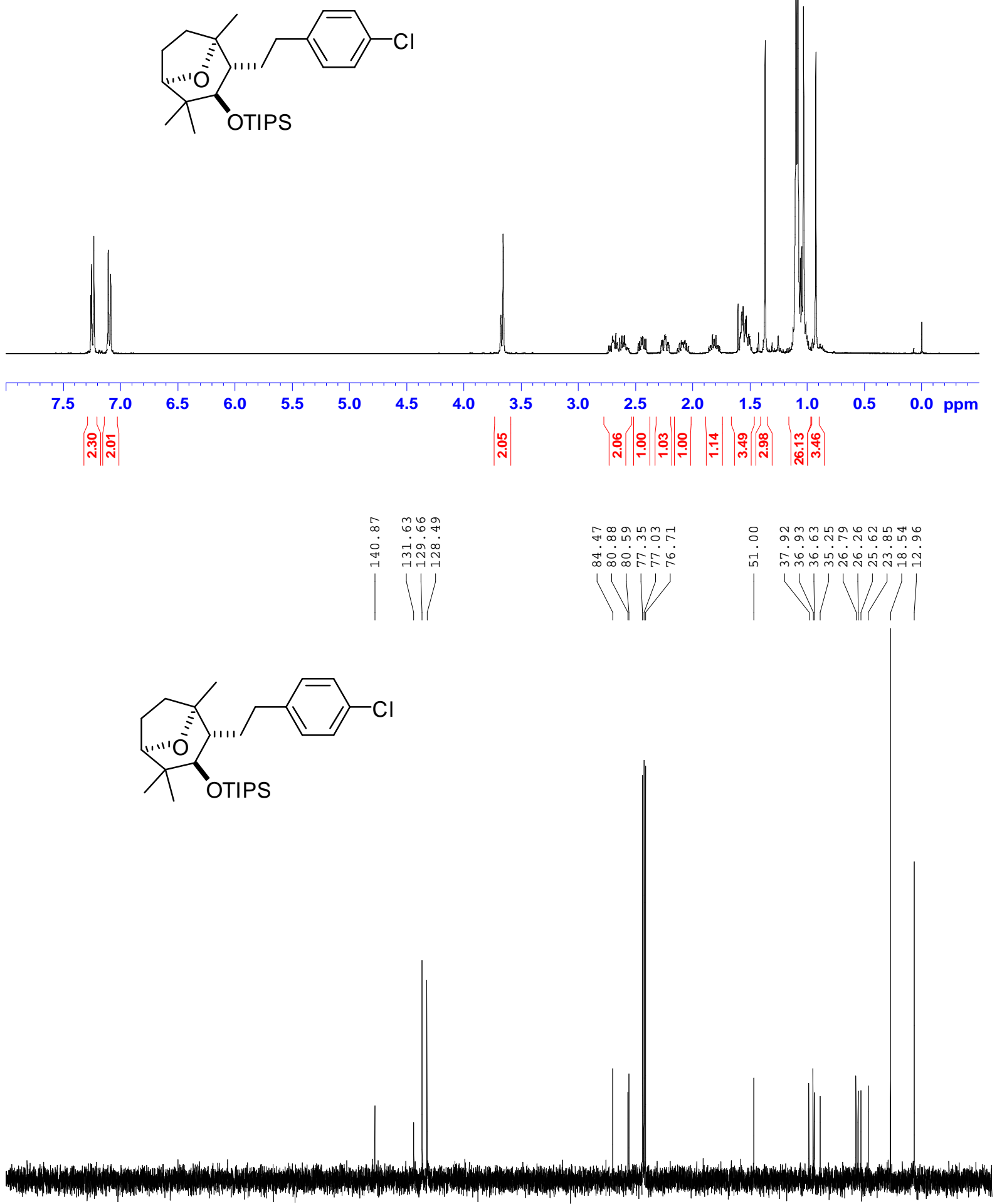

$\begin{array}{lllllllllllllllllllllll}220 & 210 & 200 & 190 & 180 & 170 & 160 & 150 & 140 & 130 & 120 & 110 & 100 & 90 & 80 & 70 & 60 & 50 & 40 & 30 & 20 & 10 & \text { ppm }\end{array}$ 

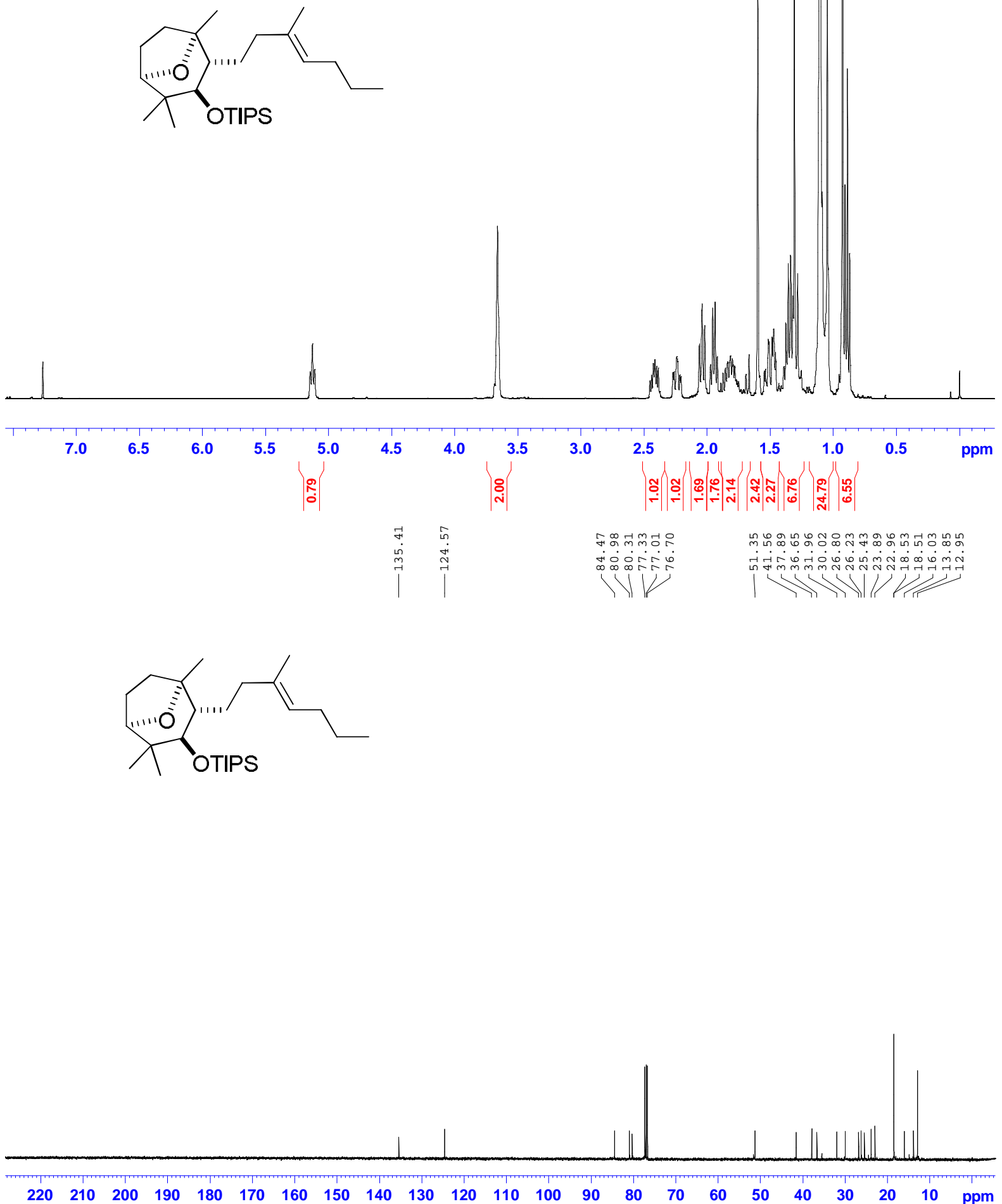

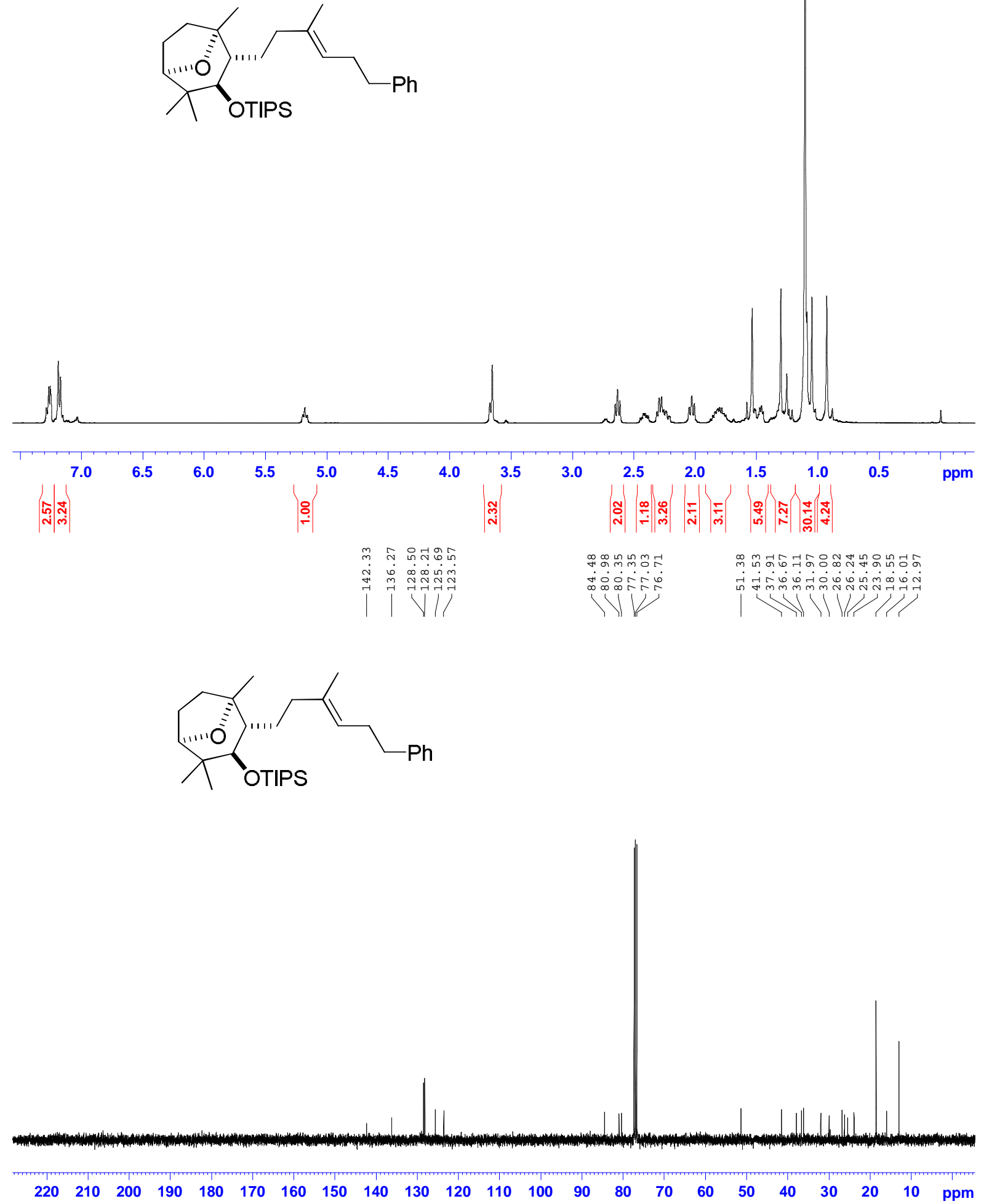

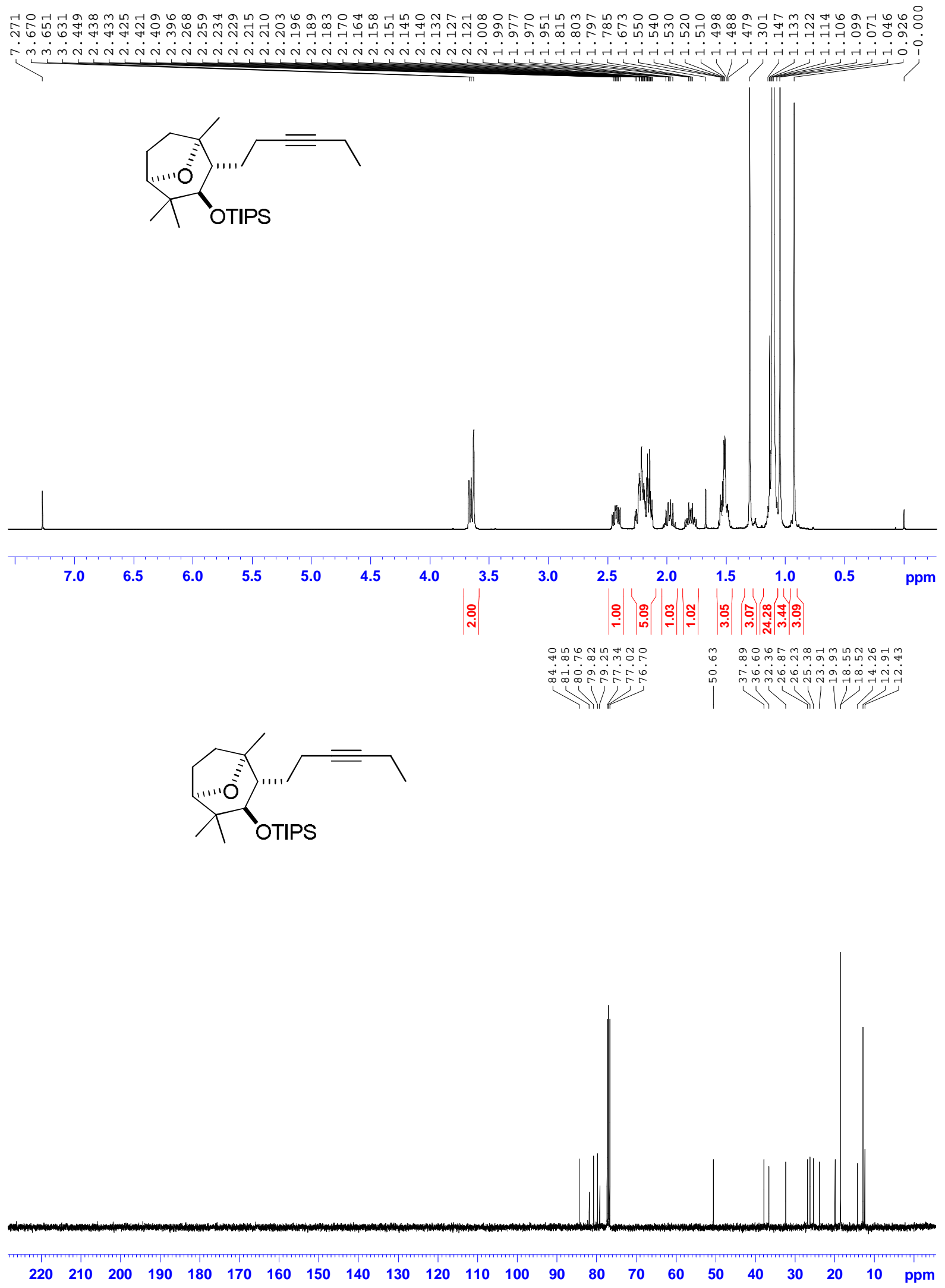

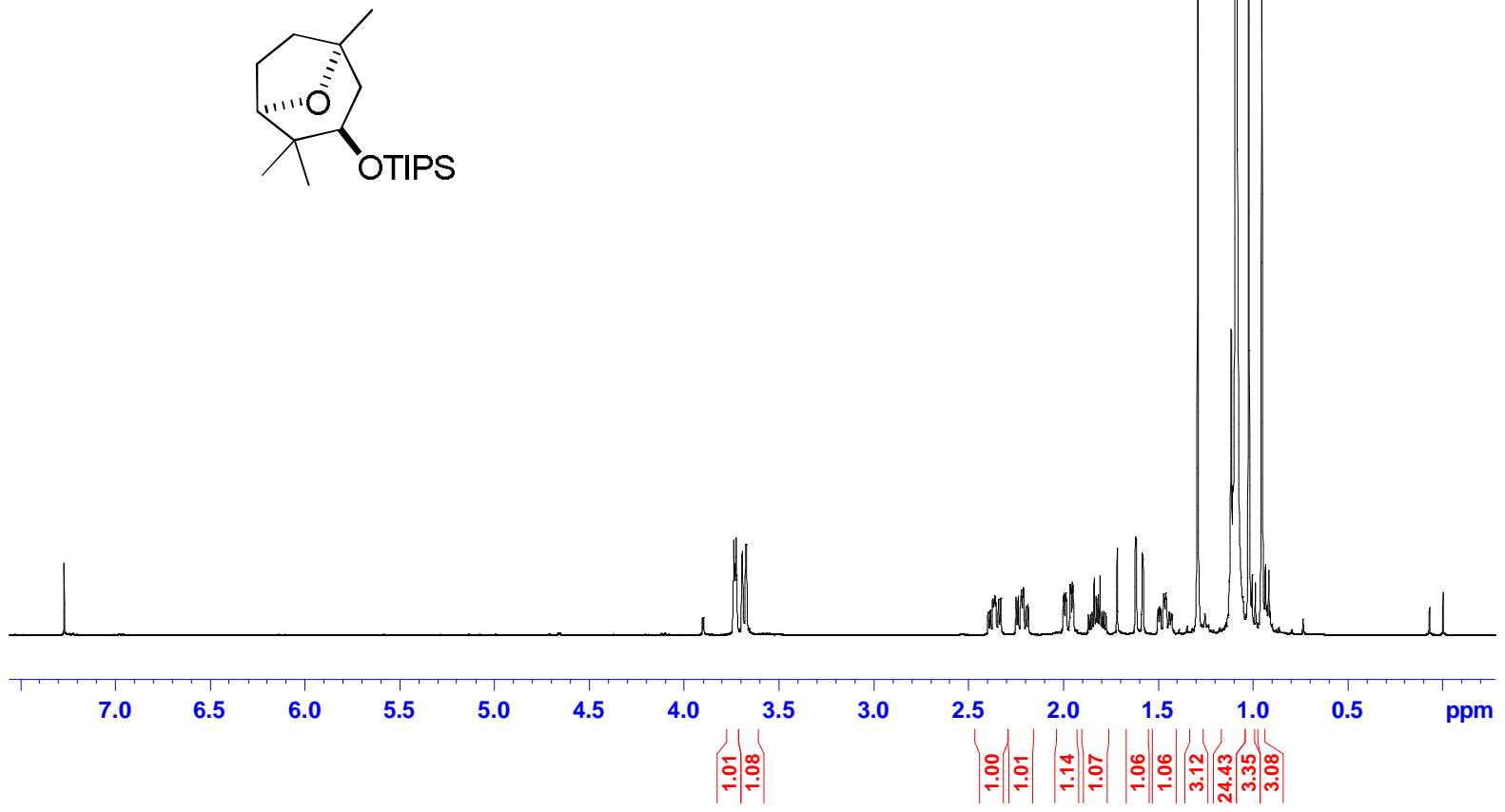

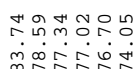

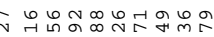

(1)

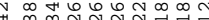

| $|W| V \mid$
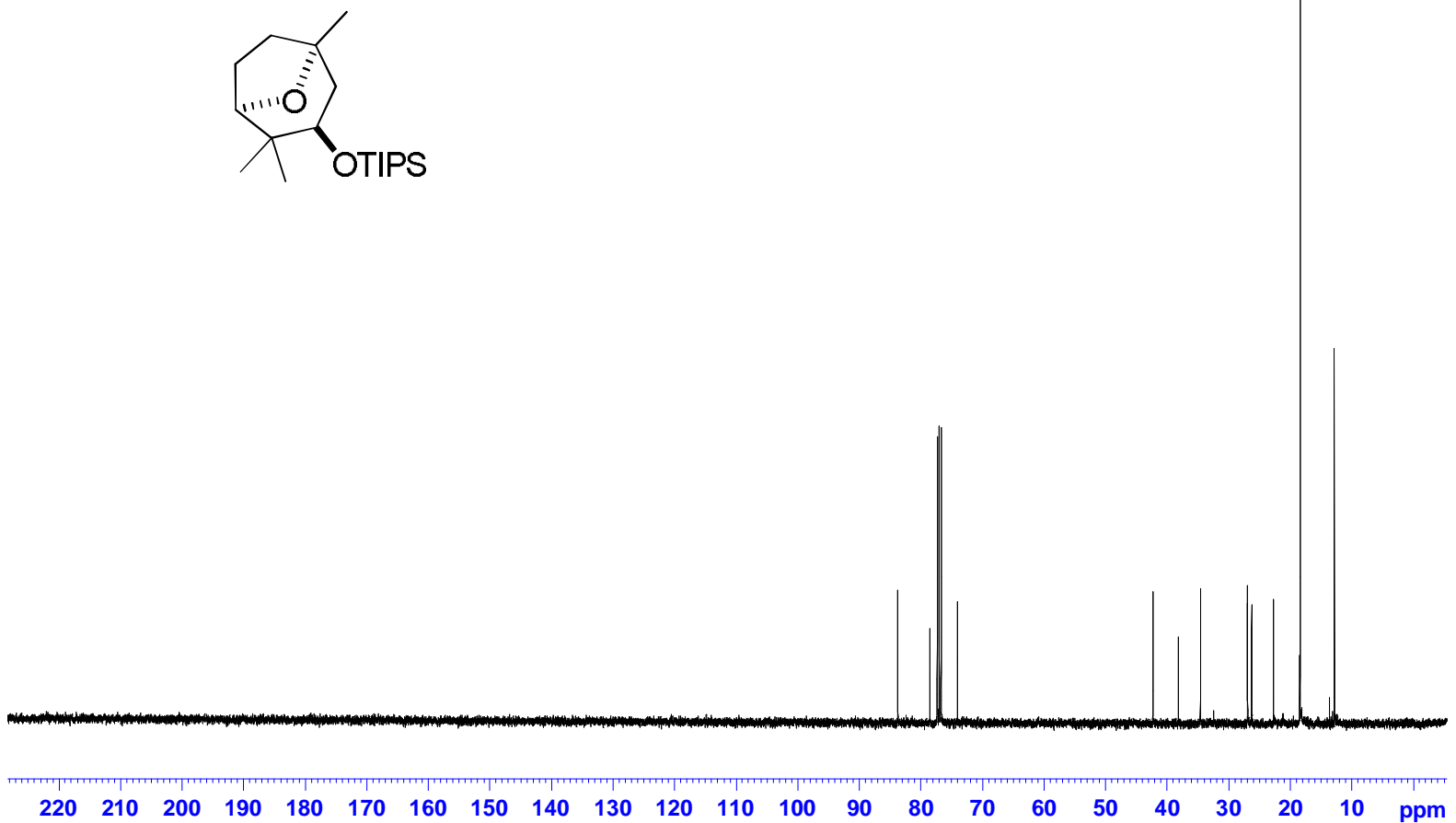

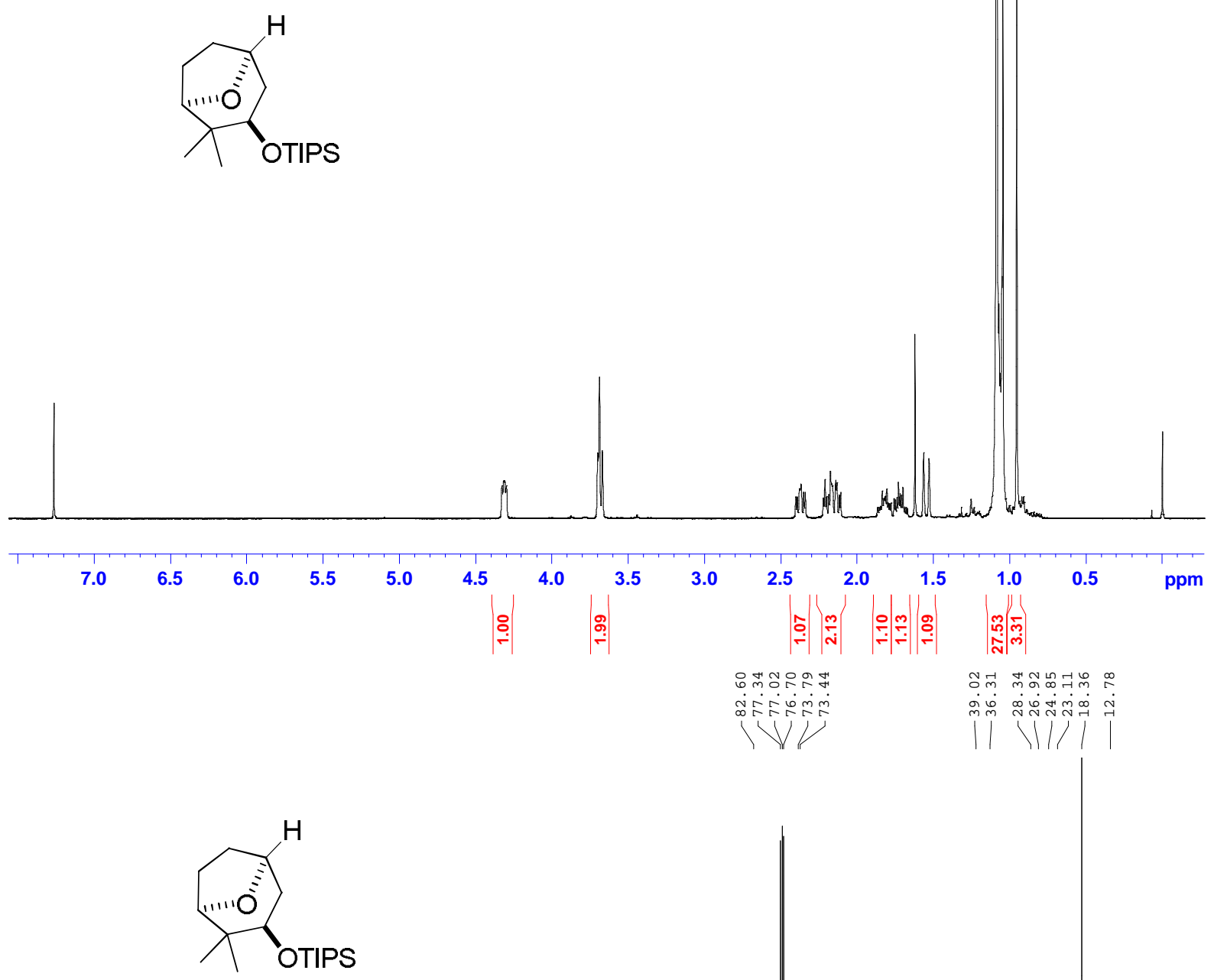

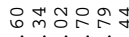

ind

WK 

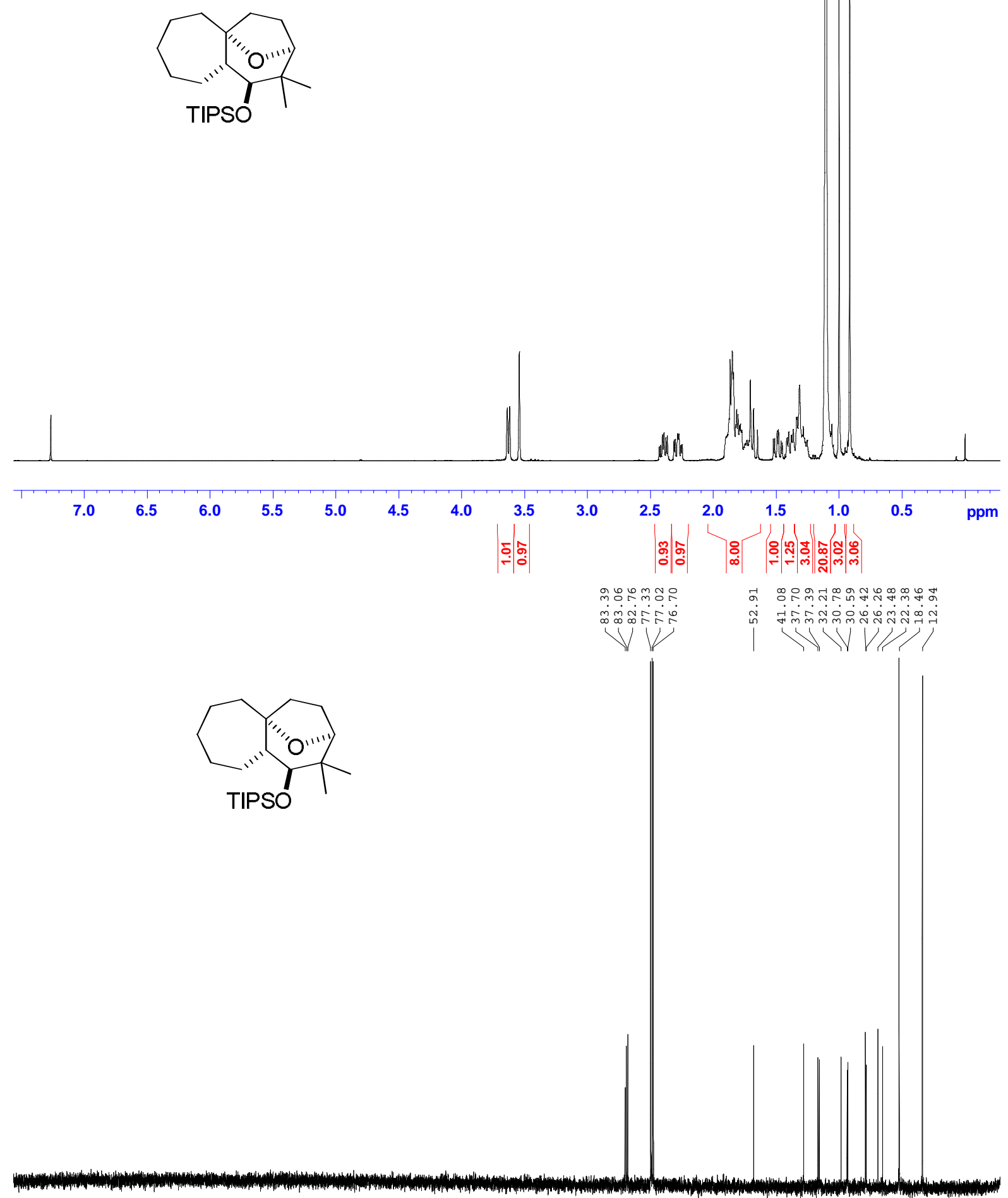

$\begin{array}{llllllllllllllllllllllll}220 & 210 & 200 & 190 & 180 & 170 & 160 & 150 & 140 & 130 & 120 & 110 & 100 & 90 & 80 & 70 & 60 & 50 & 40 & 30 & 20 & 10 & \text { ppm }\end{array}$ 


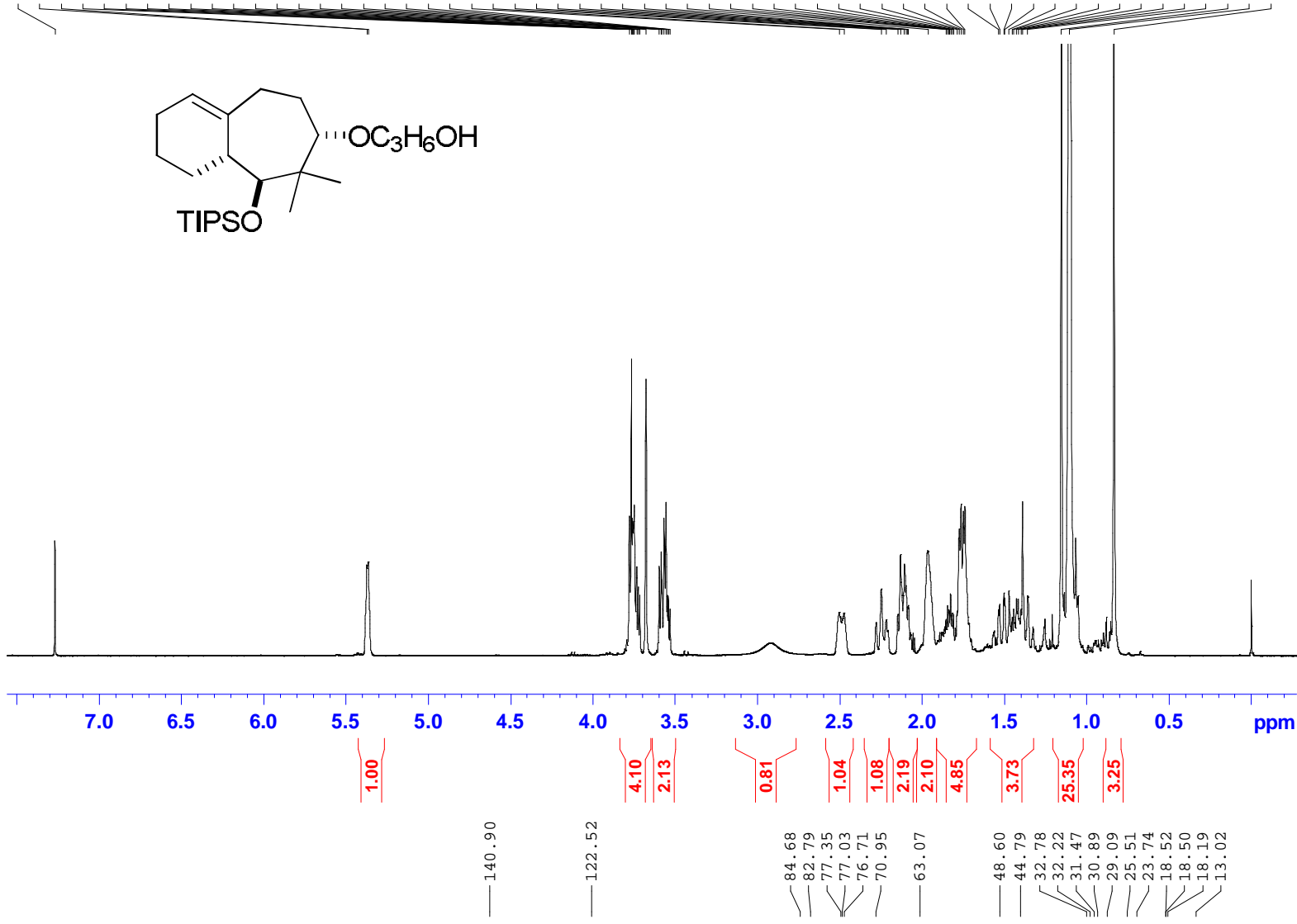<smiles>CCO[C@H]1CCC2=CCCC[C@H]2C(C)(C)C1OCO</smiles>

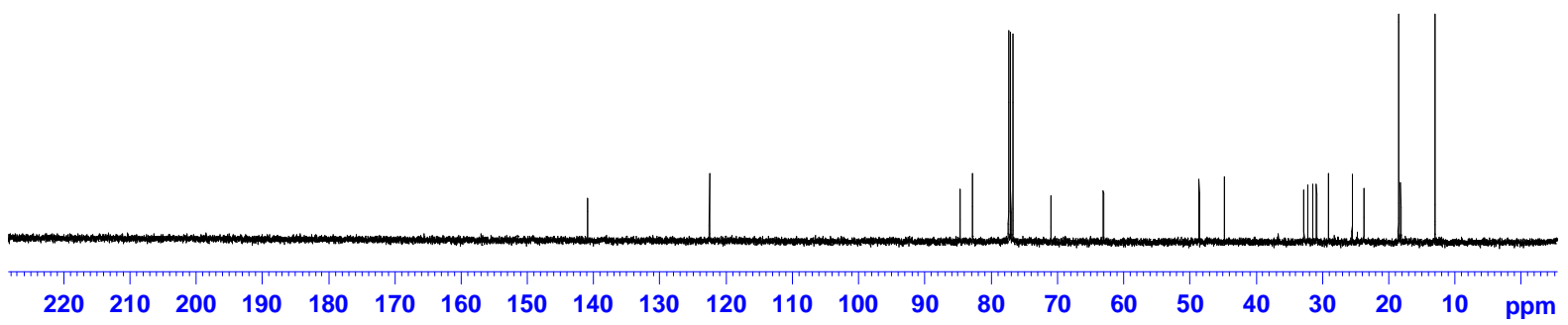



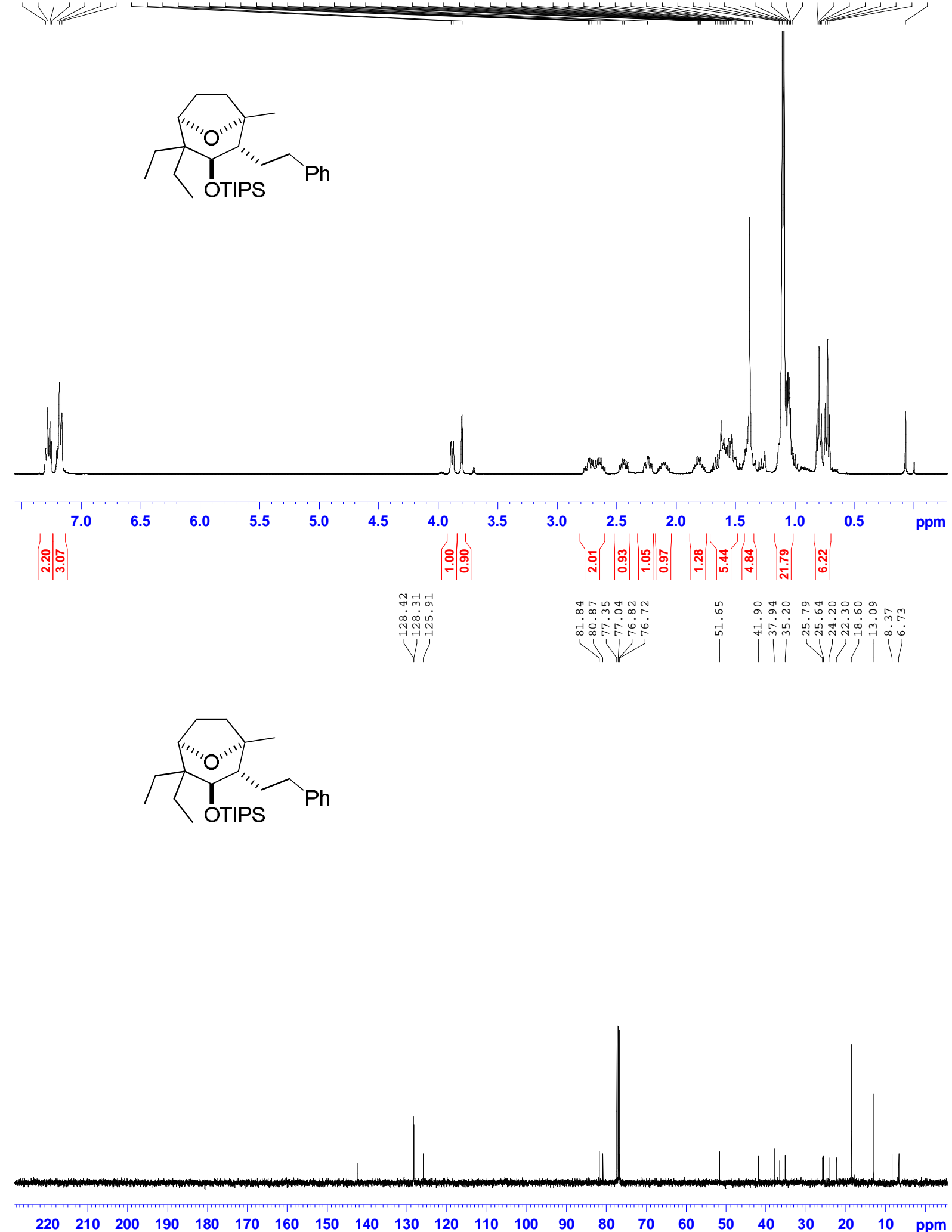


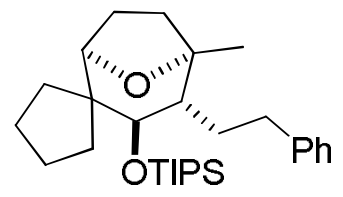
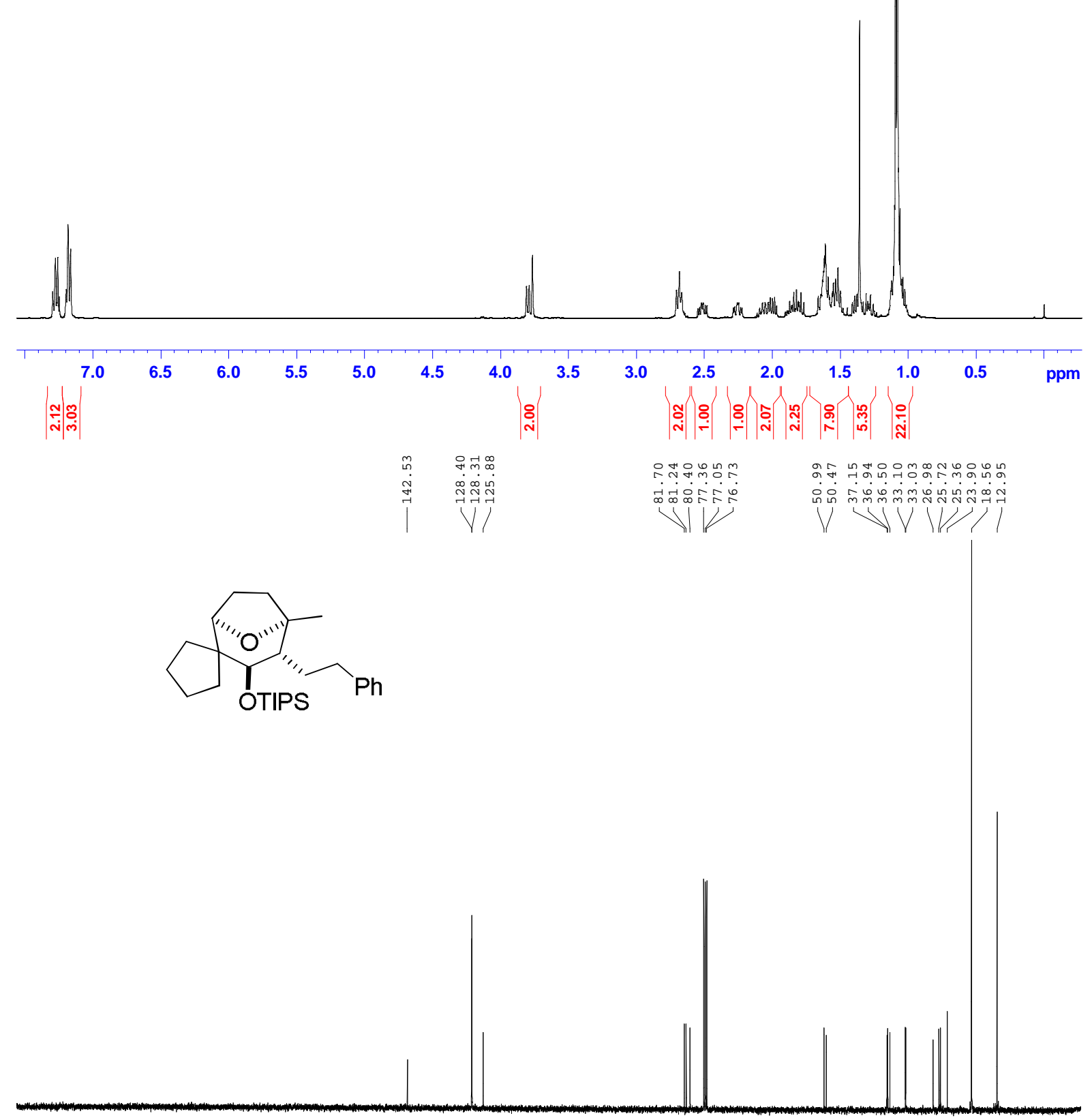

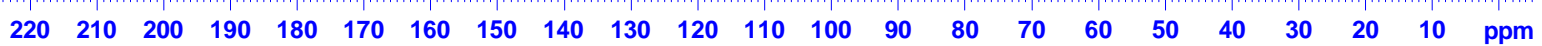



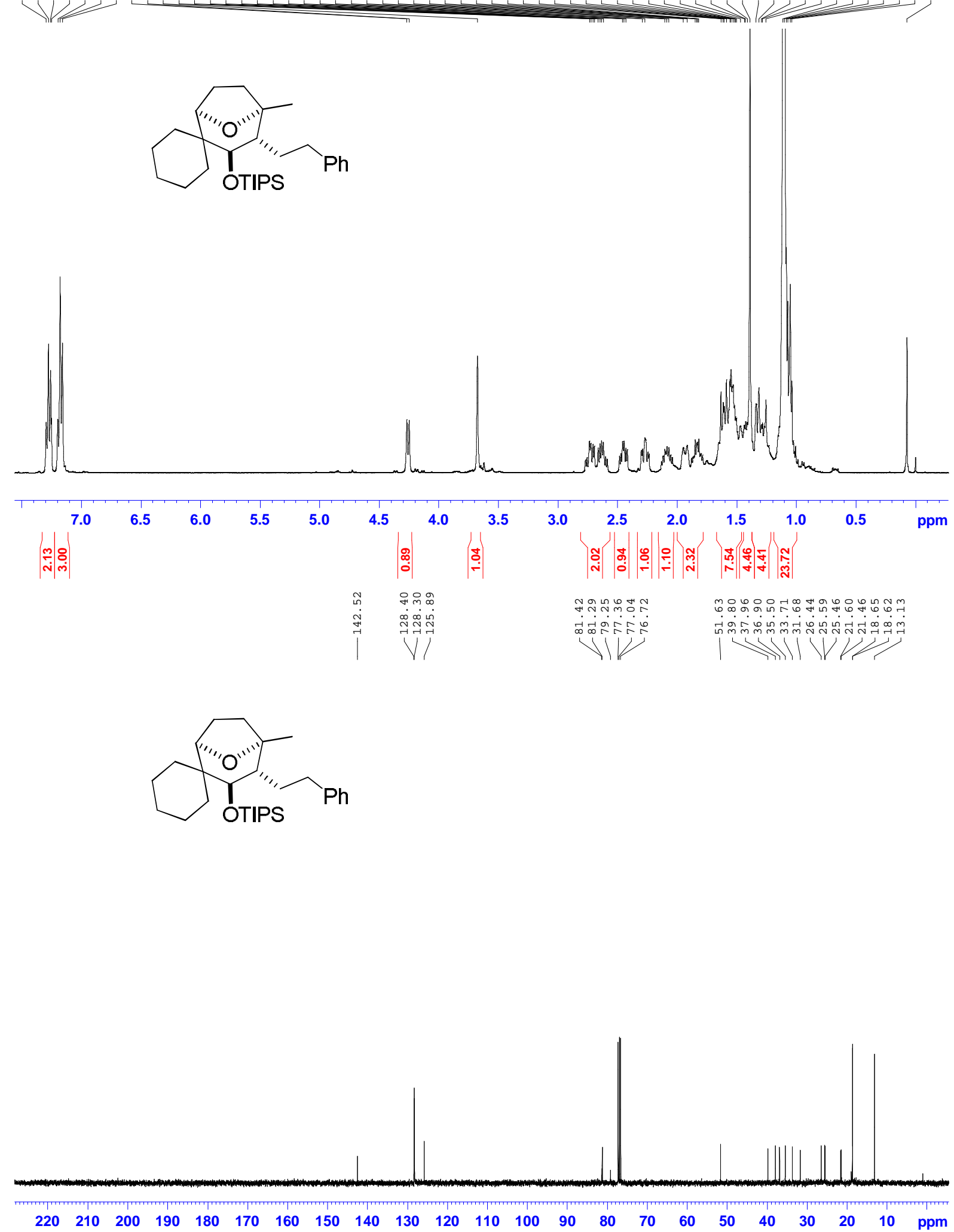
<smiles>CC12CCC(O1)[C@@H](Cc1ccccc1)[C@H]2CCc1ccccc1</smiles>
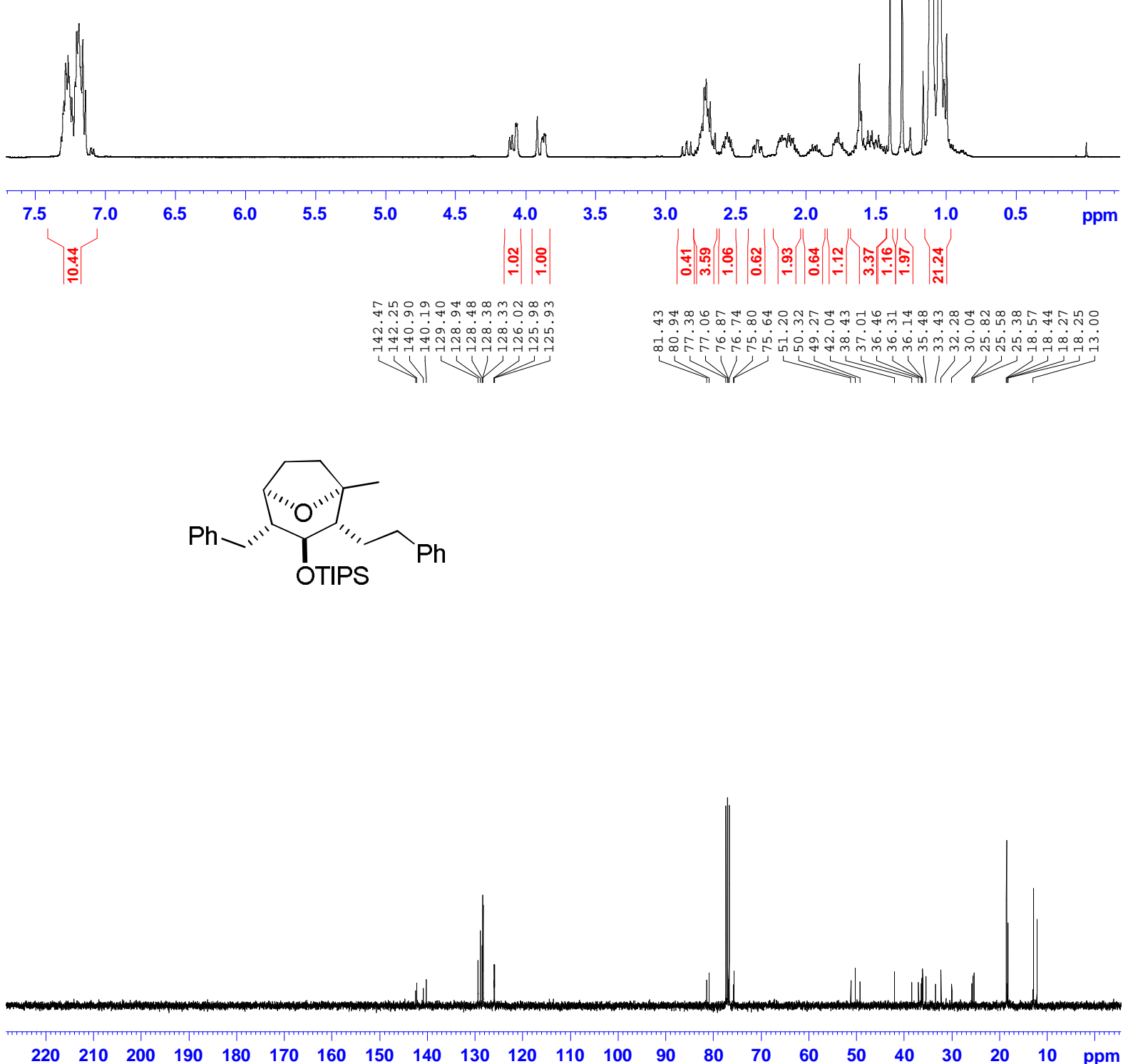


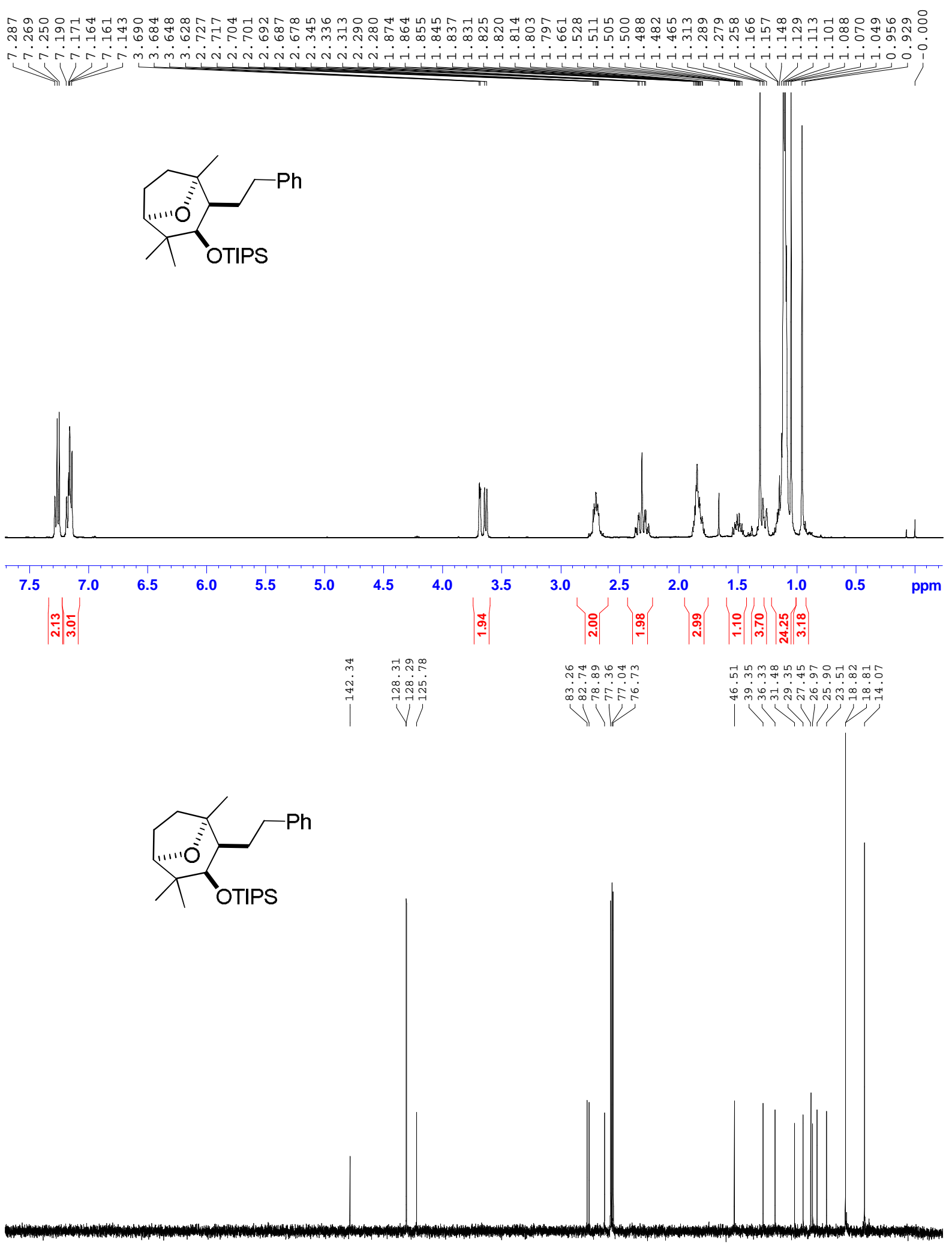

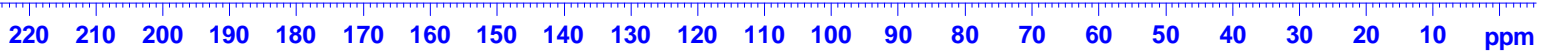



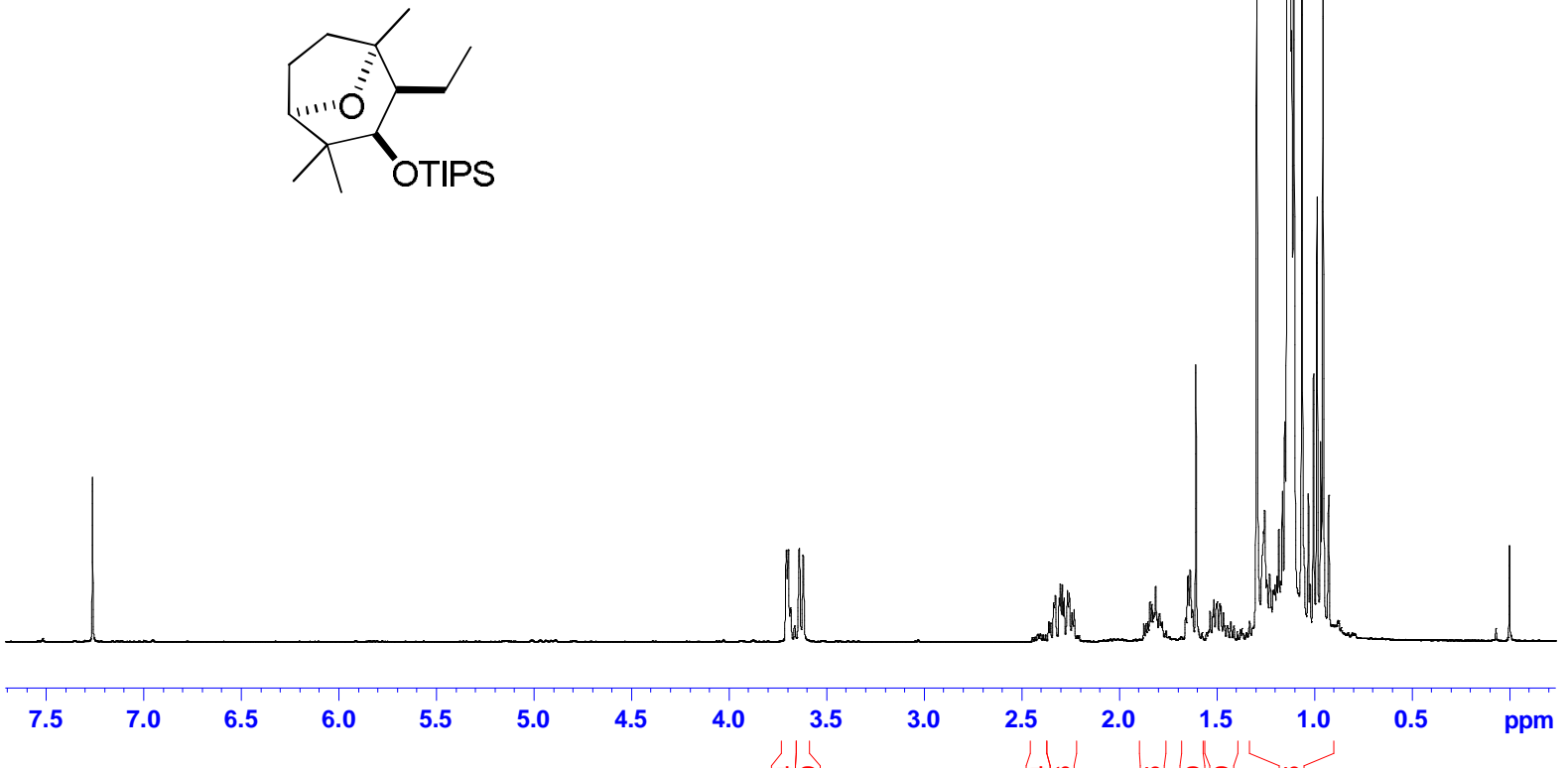

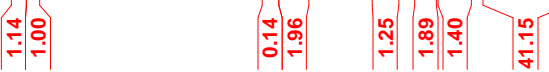

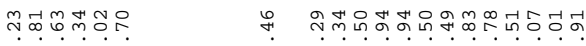

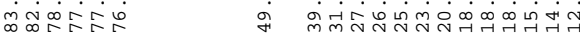

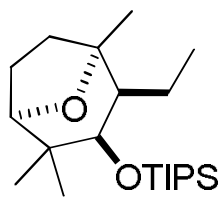



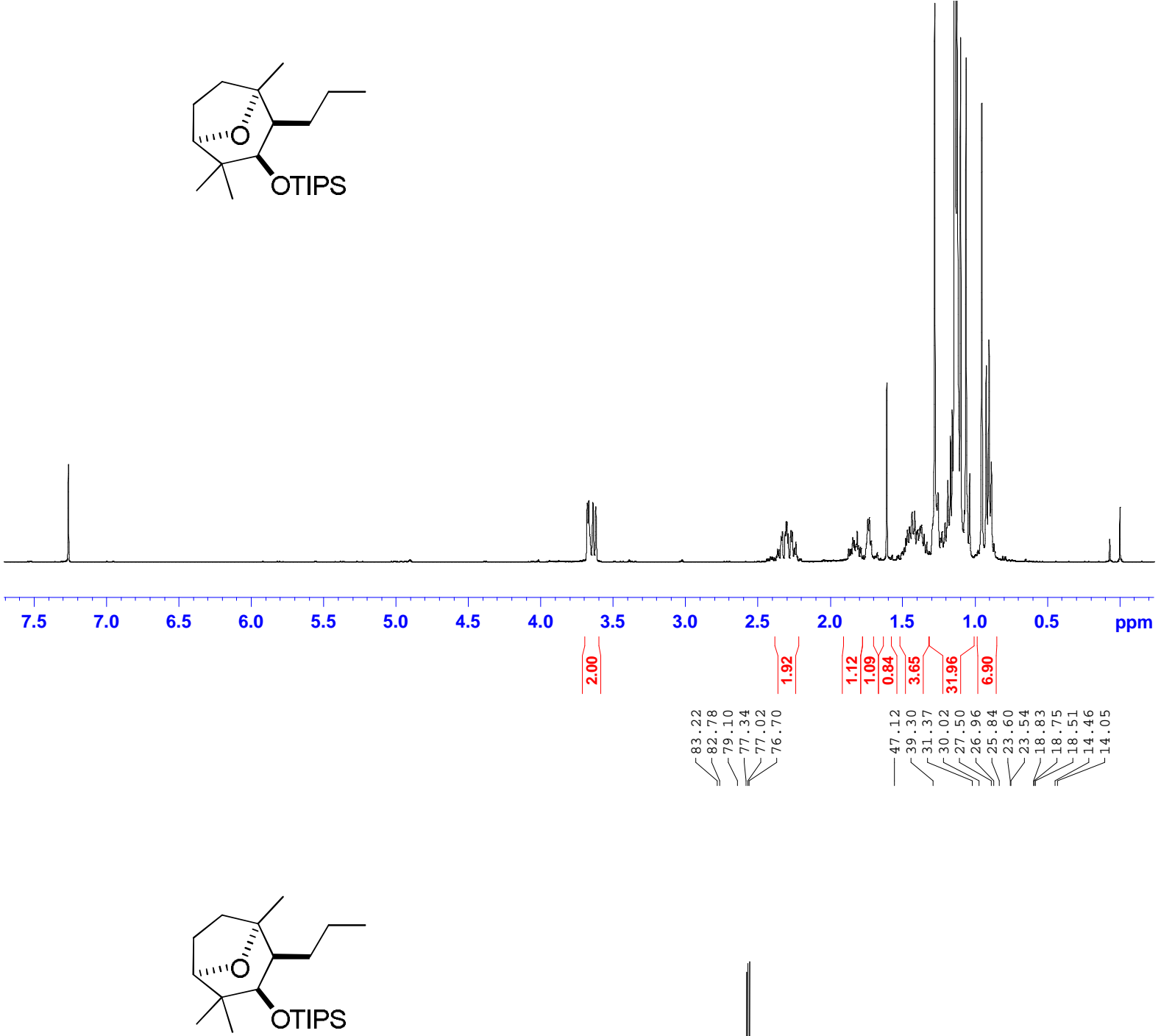

$\begin{array}{lllllllllllllllllllllll}220 & 210 & 200 & 190 & 180 & 170 & 160 & 150 & 140 & 130 & 120 & 110 & 100 & 90 & 80 & 70 & 60 & 50 & 40 & 30 & 20 & 10 & \mathrm{ppm}\end{array}$ 

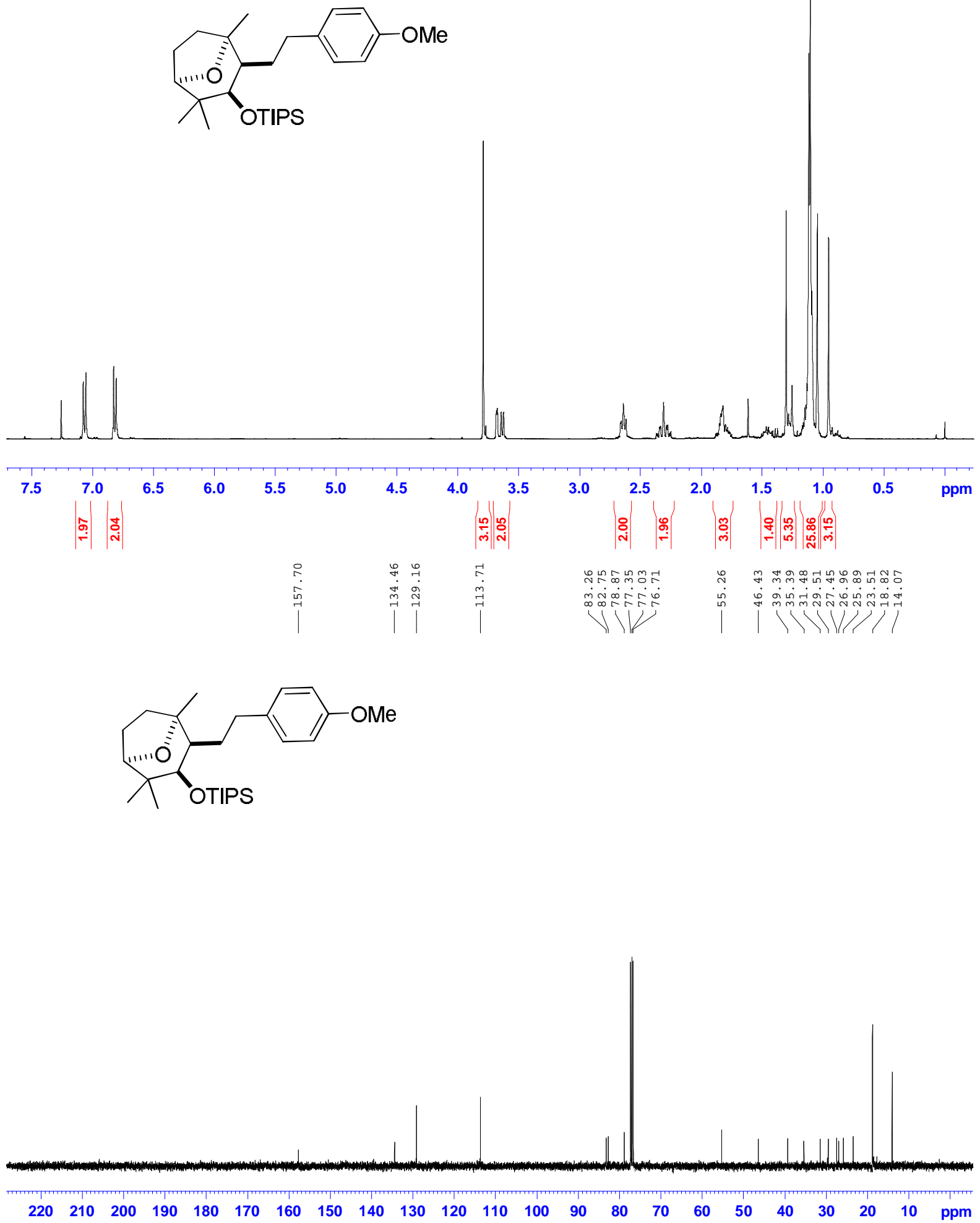

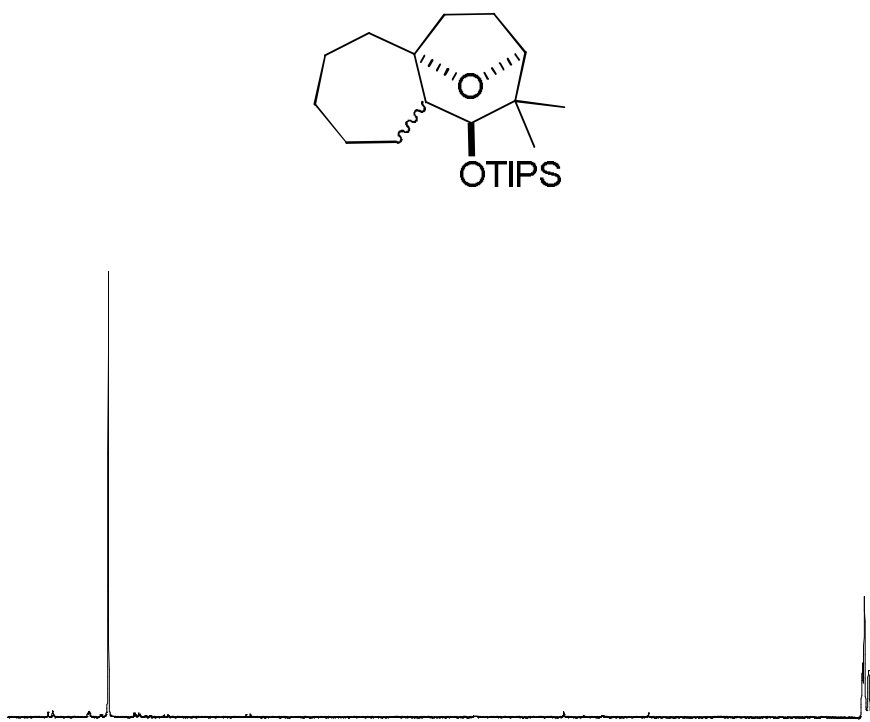

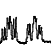
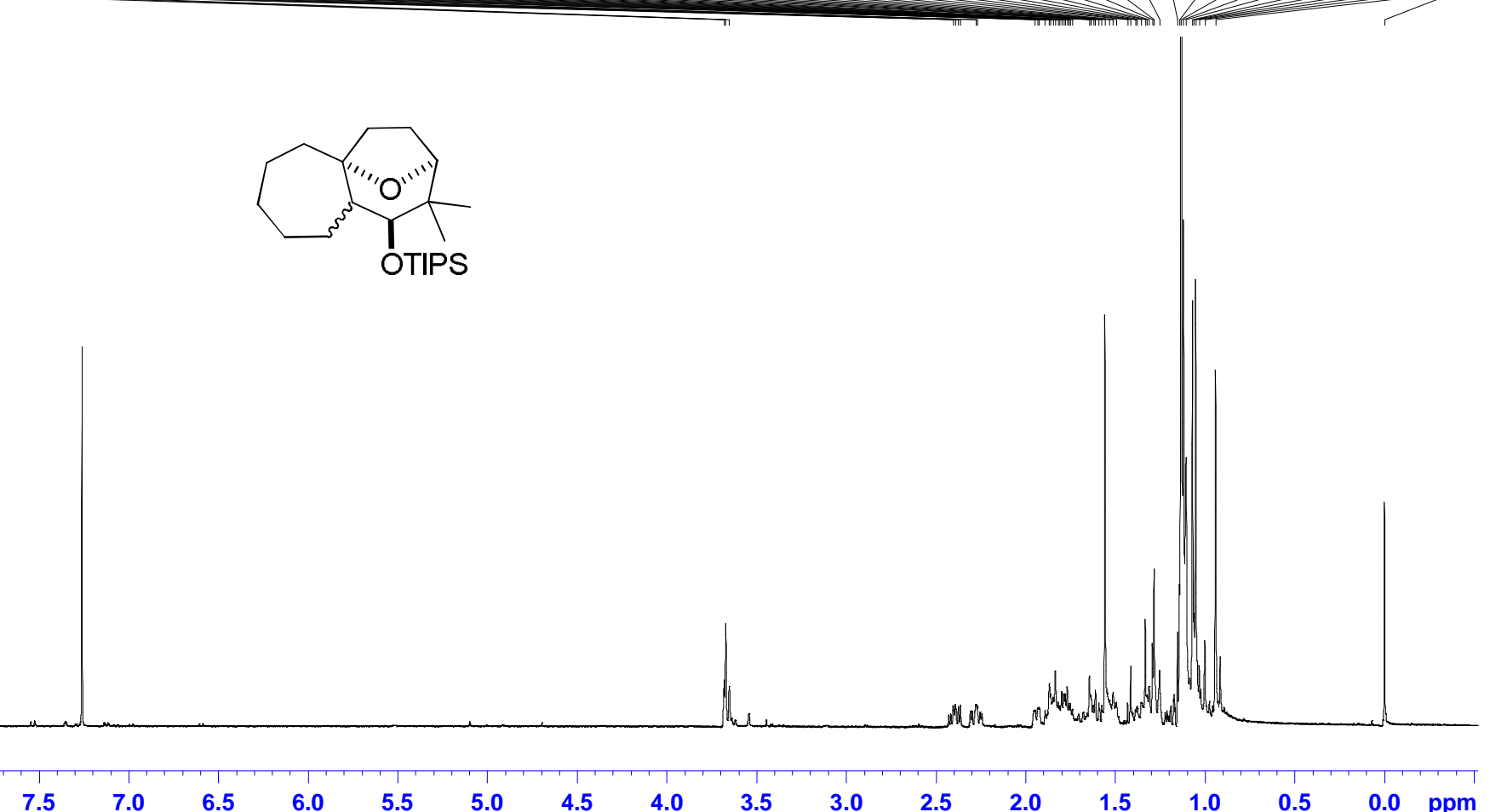

7.5 7.0

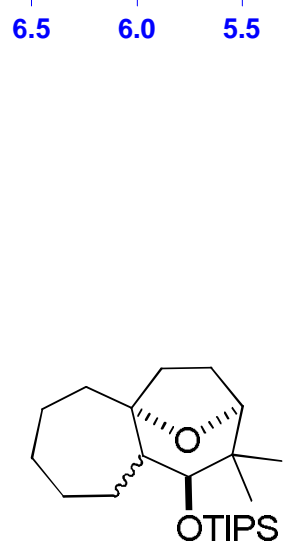

$5.0 \quad 4.5$

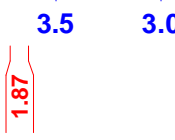

2.5

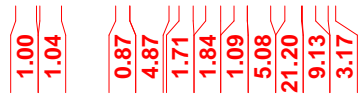
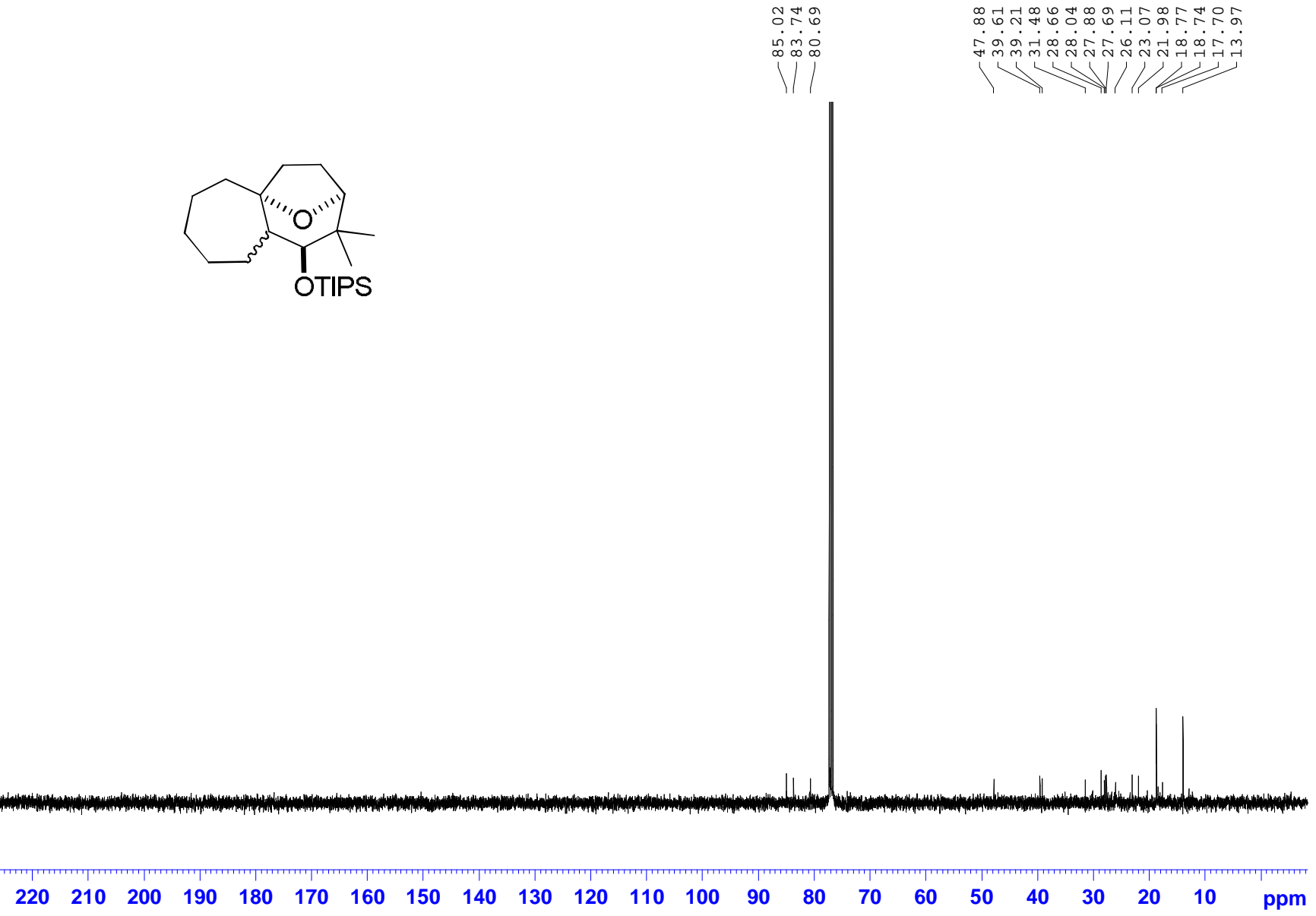

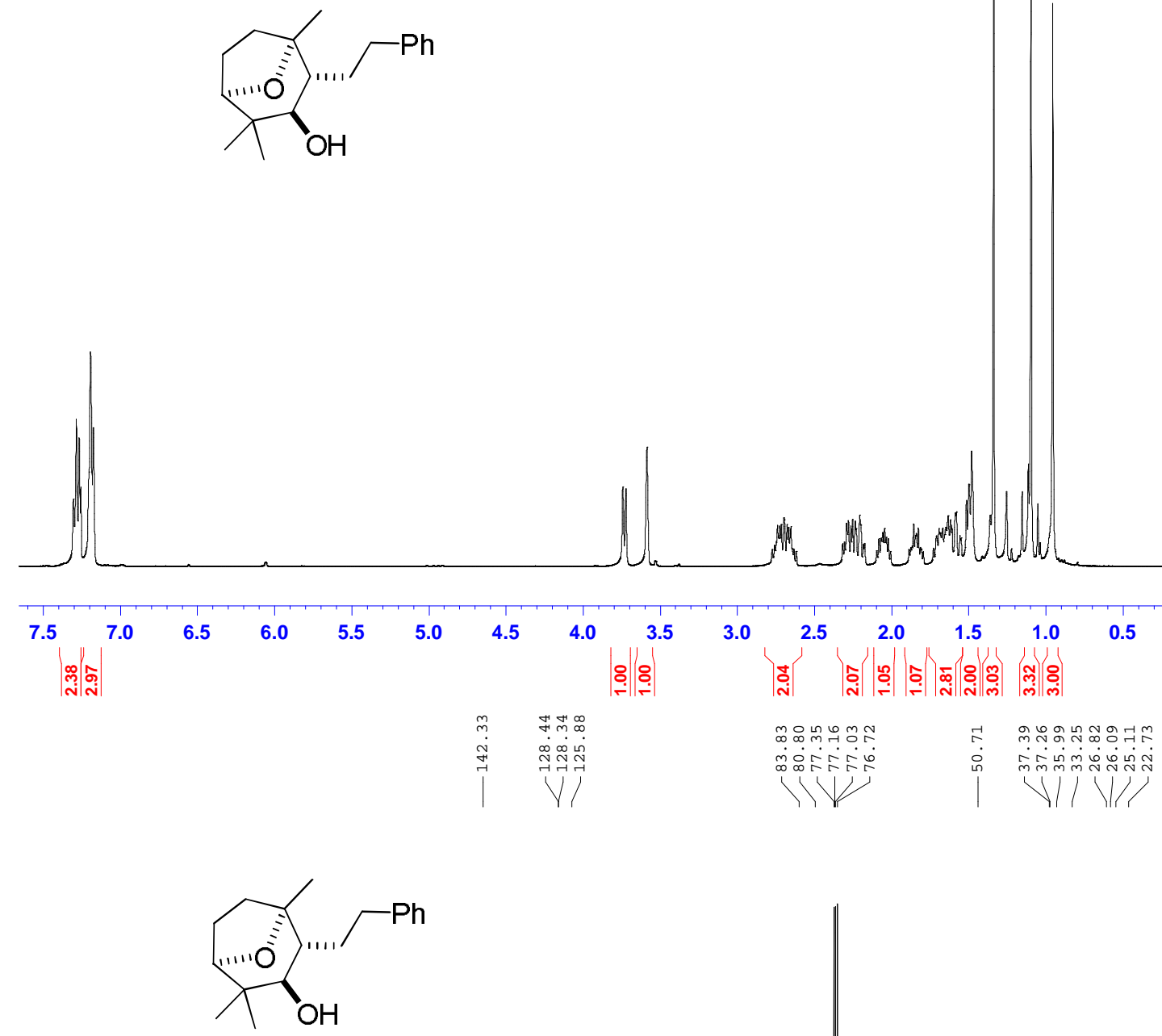

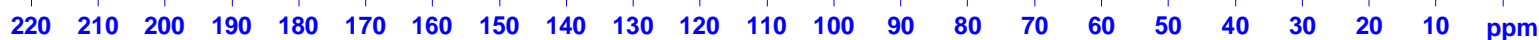


<smiles>COc1ccc(CCC2C(O)C(C)(C)[C@@H]3CC[C@@]2(C)O3)cc1</smiles>

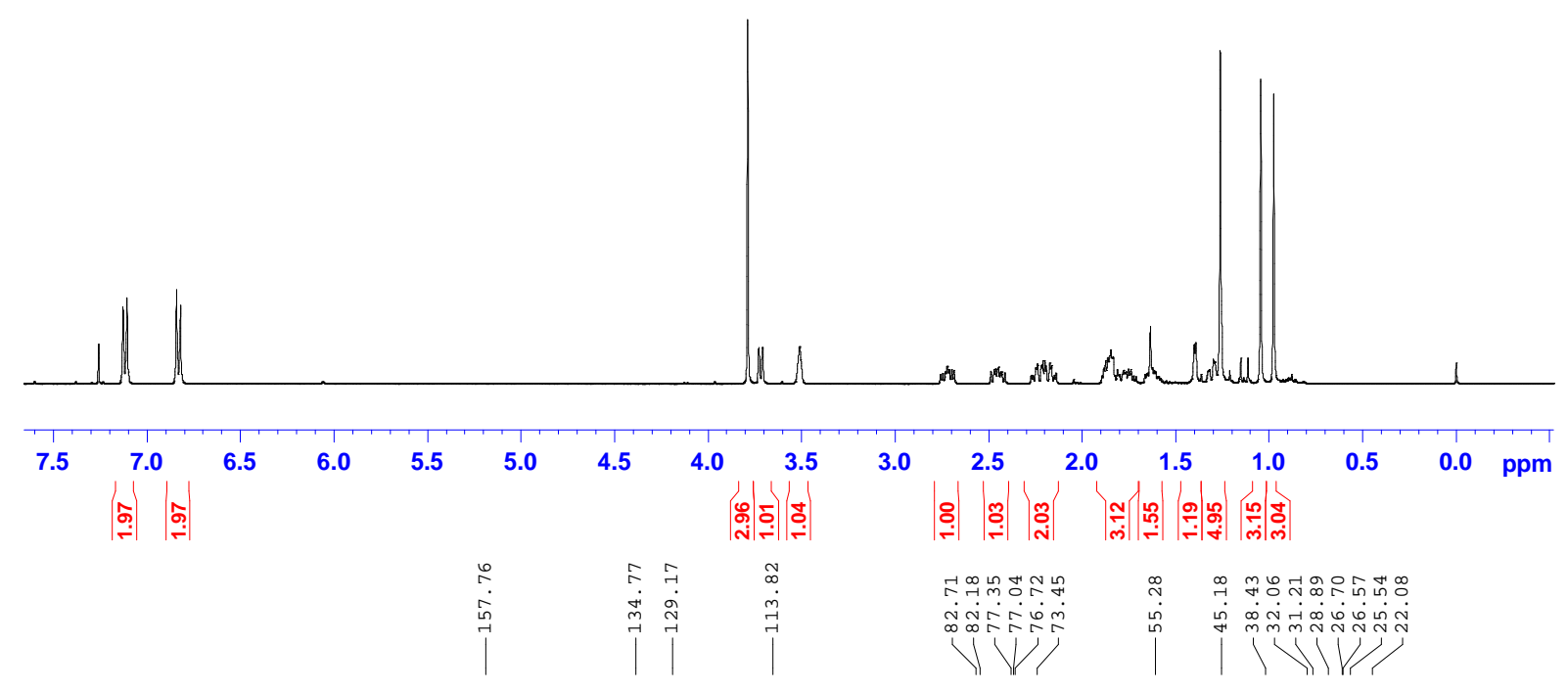<smiles>COc1ccc(CCC2C(O)[C@]3(C)CC[C@@H]2OC3(C)C)cc1</smiles>

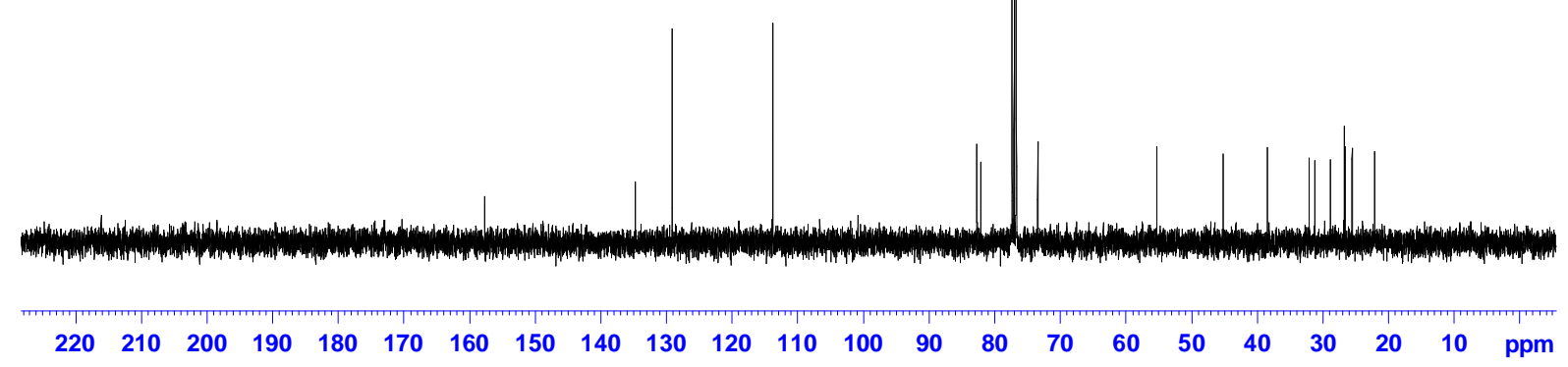



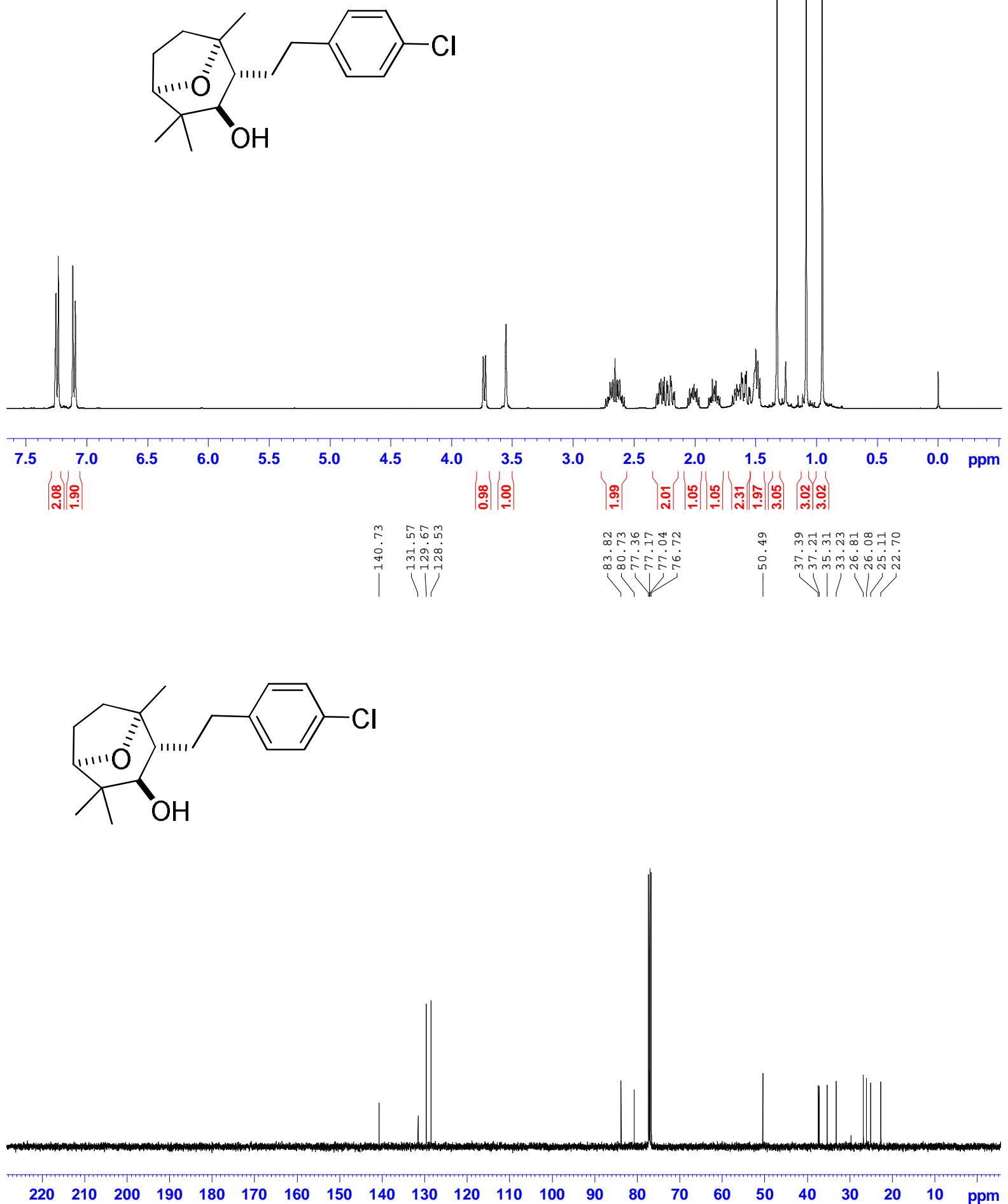
<smiles>CC(=CCCc1ccccc1)CCC1OCCCO1</smiles>

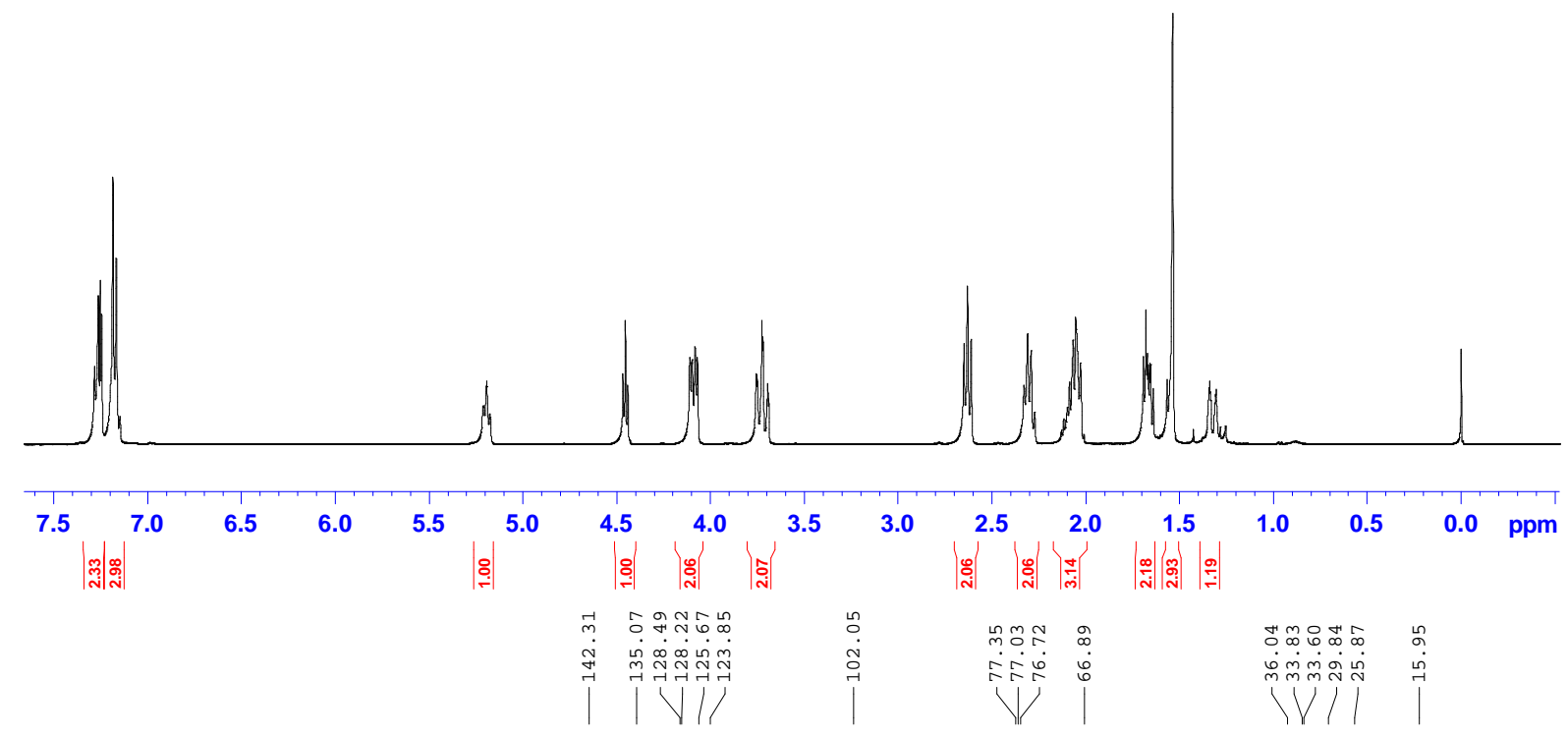<smiles>CC(=CCCc1ccccc1)CCC1OCCCO1</smiles>

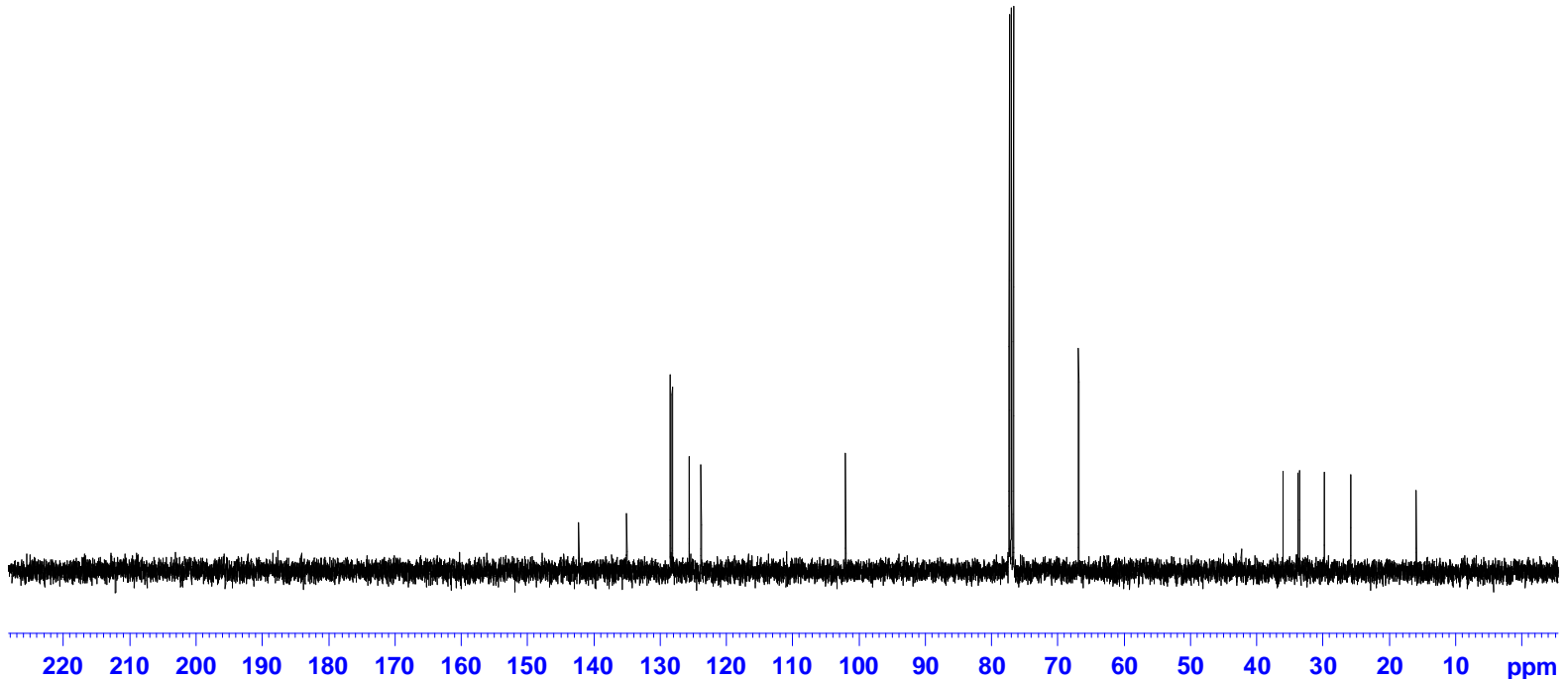




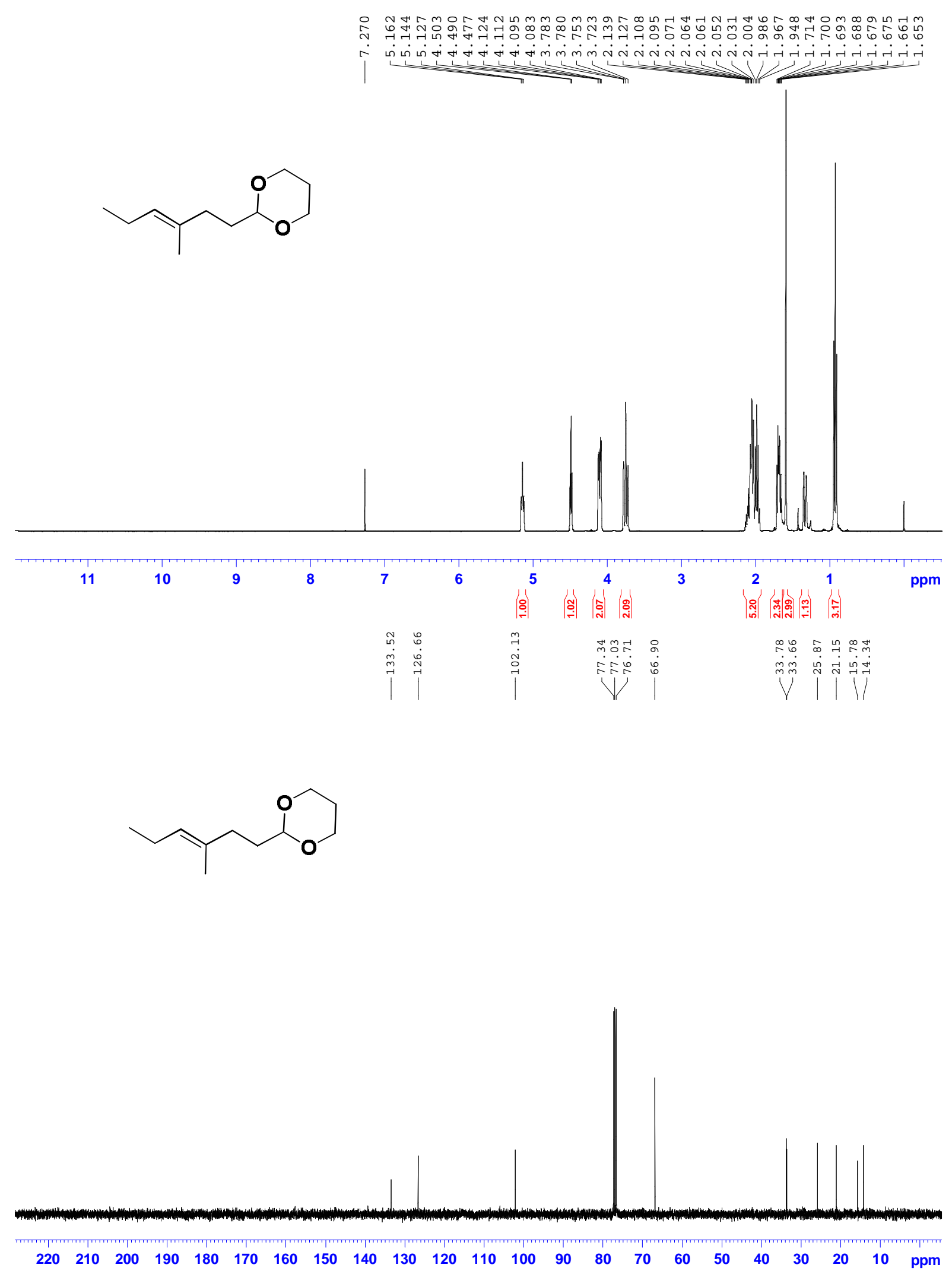




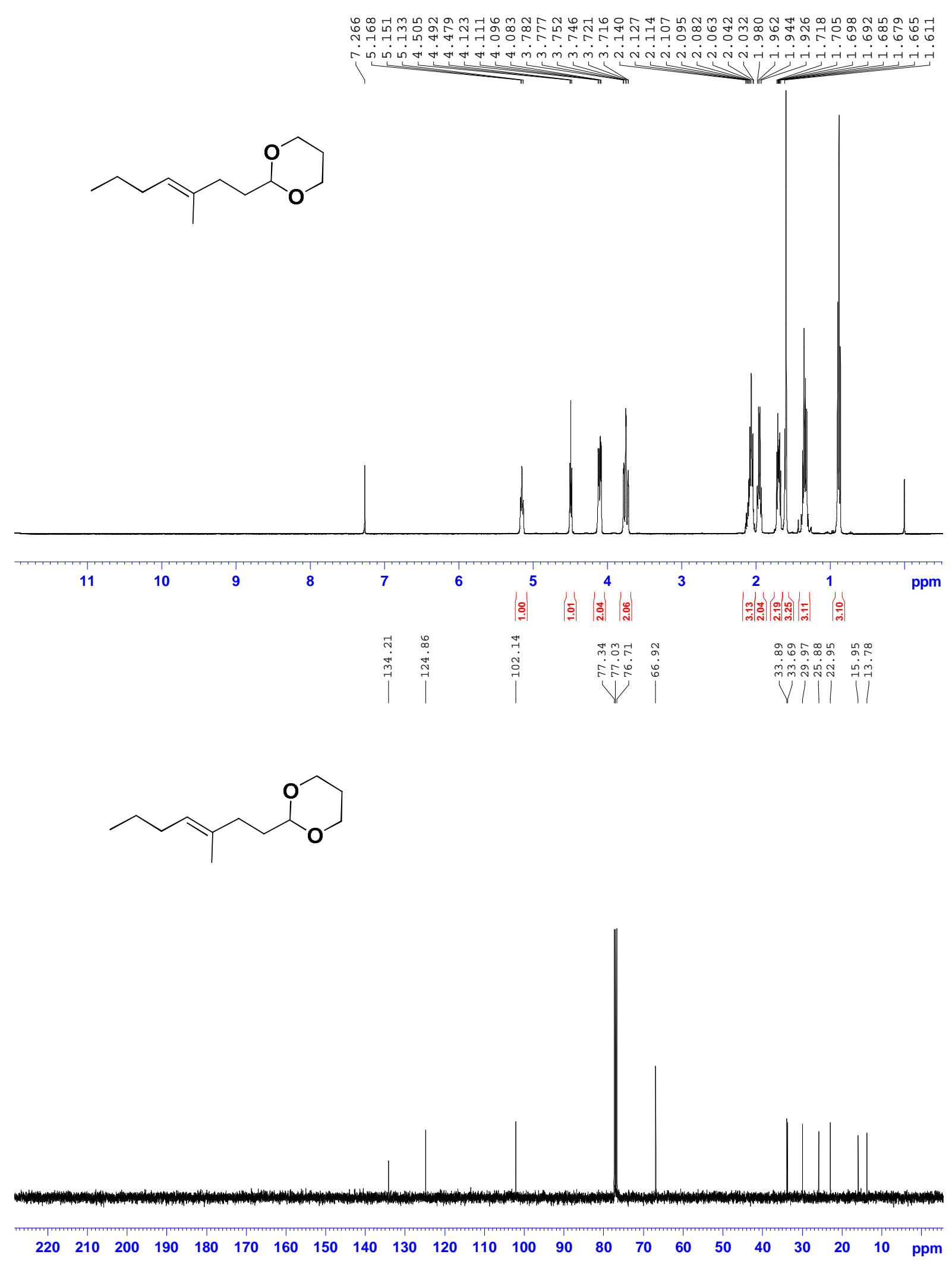




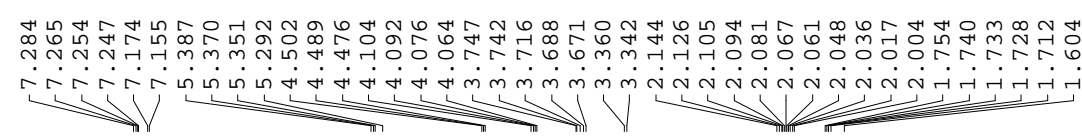

$\overbrace{}^{P}$

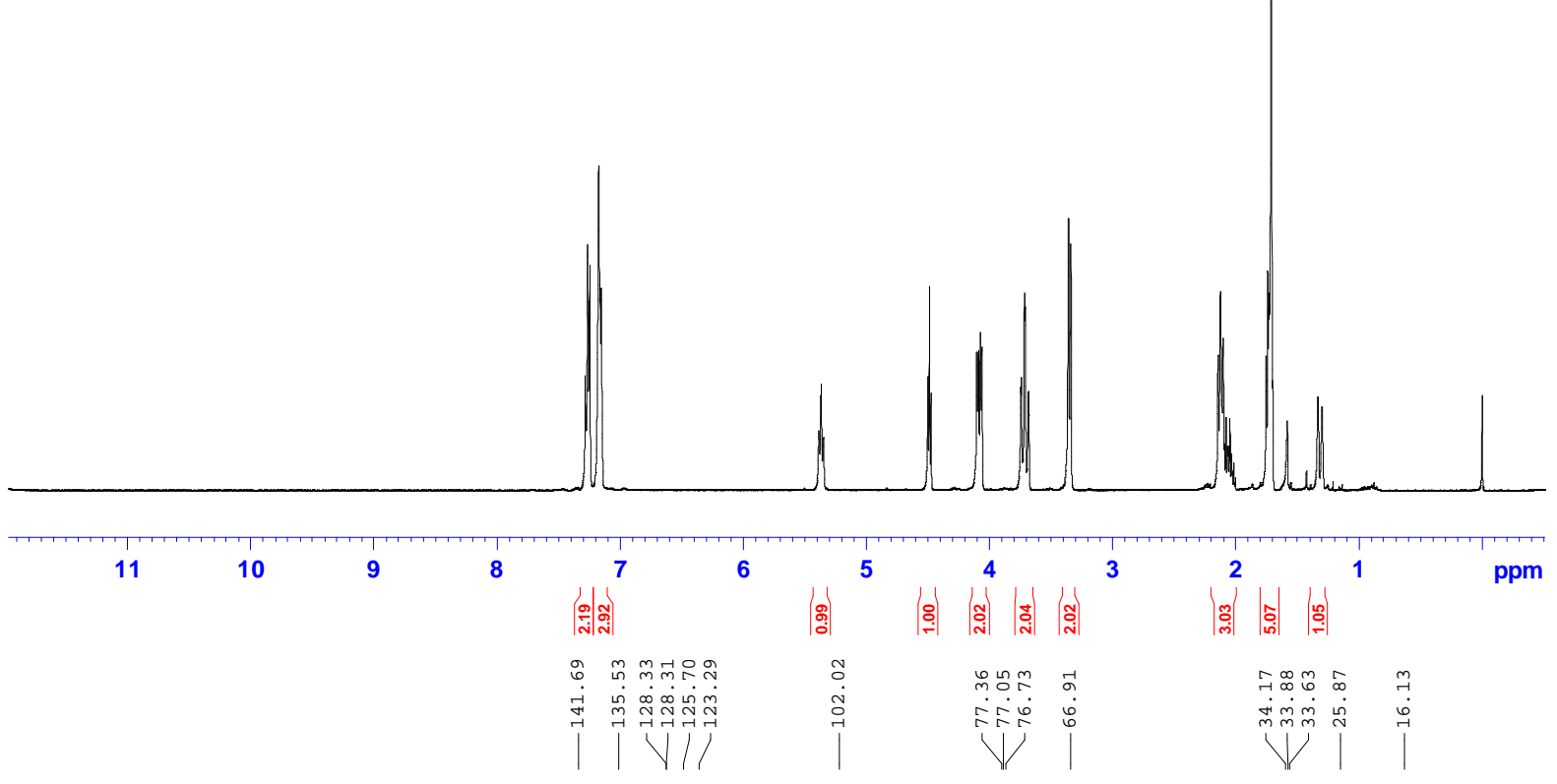<smiles>C/C(=C\Cc1ccccc1)CCC1OCCCO1</smiles>

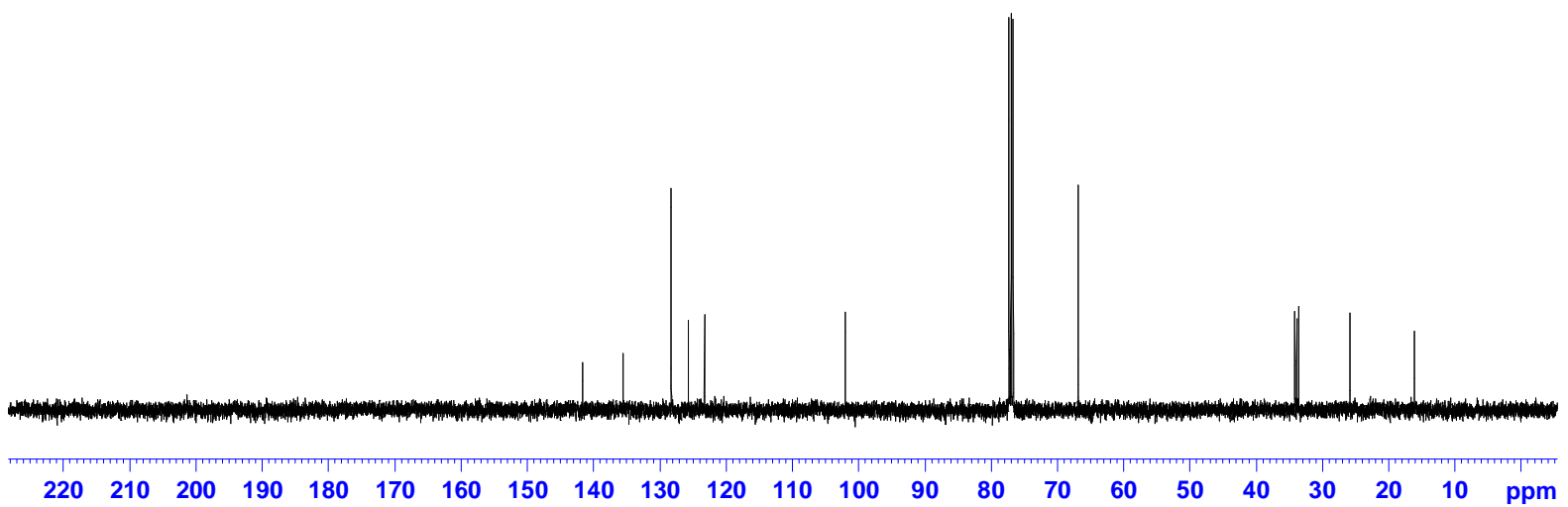




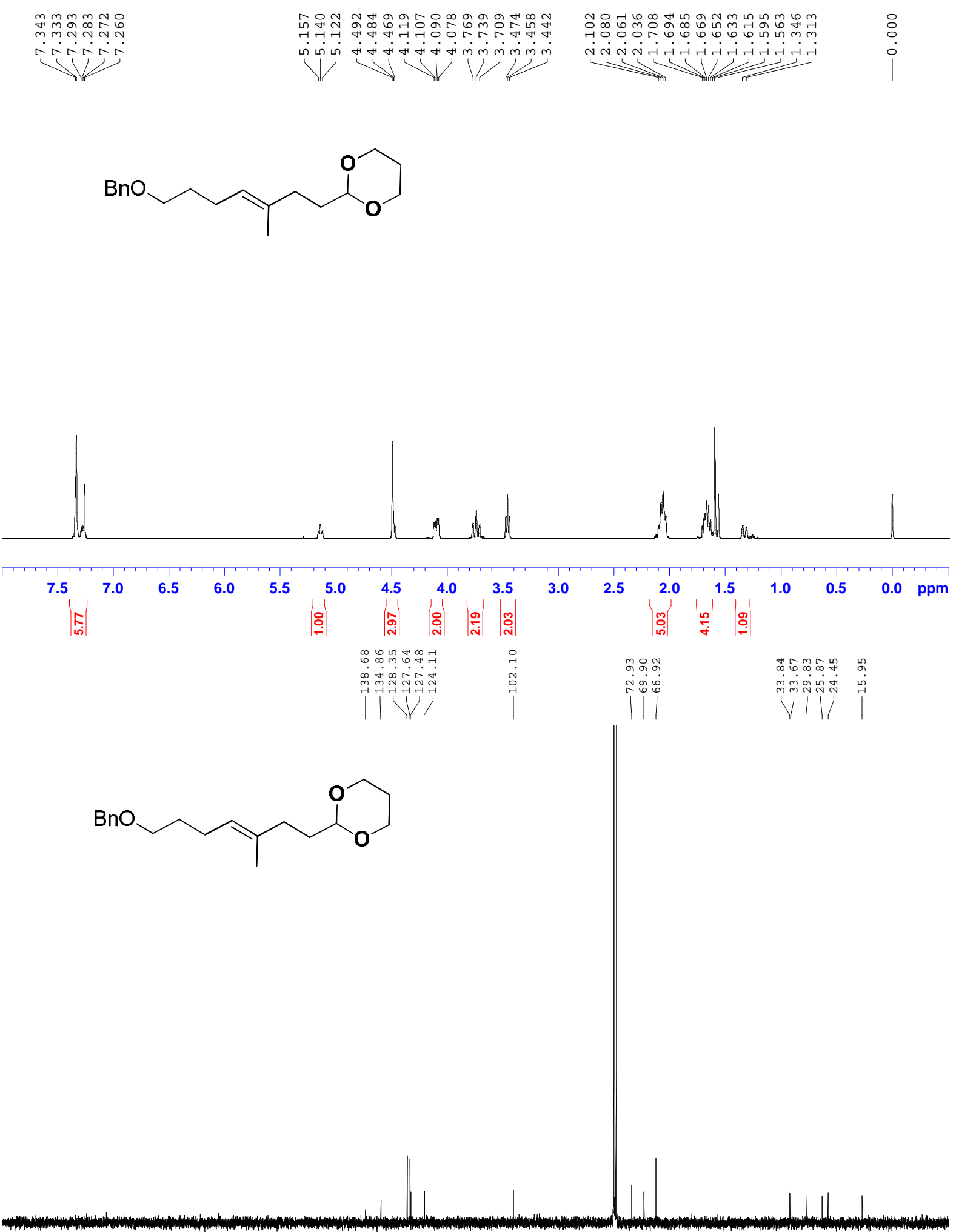

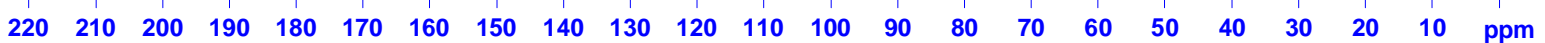



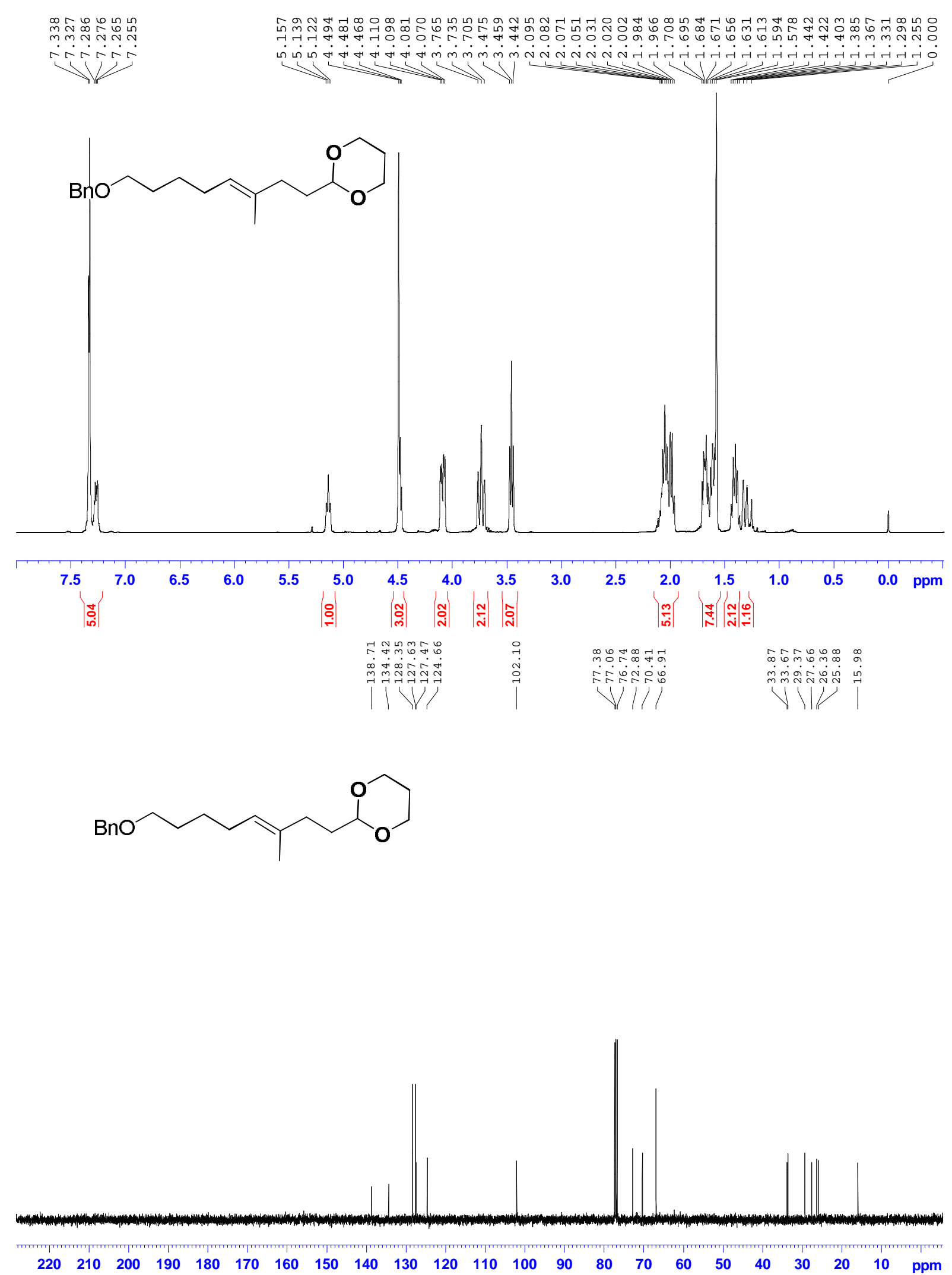

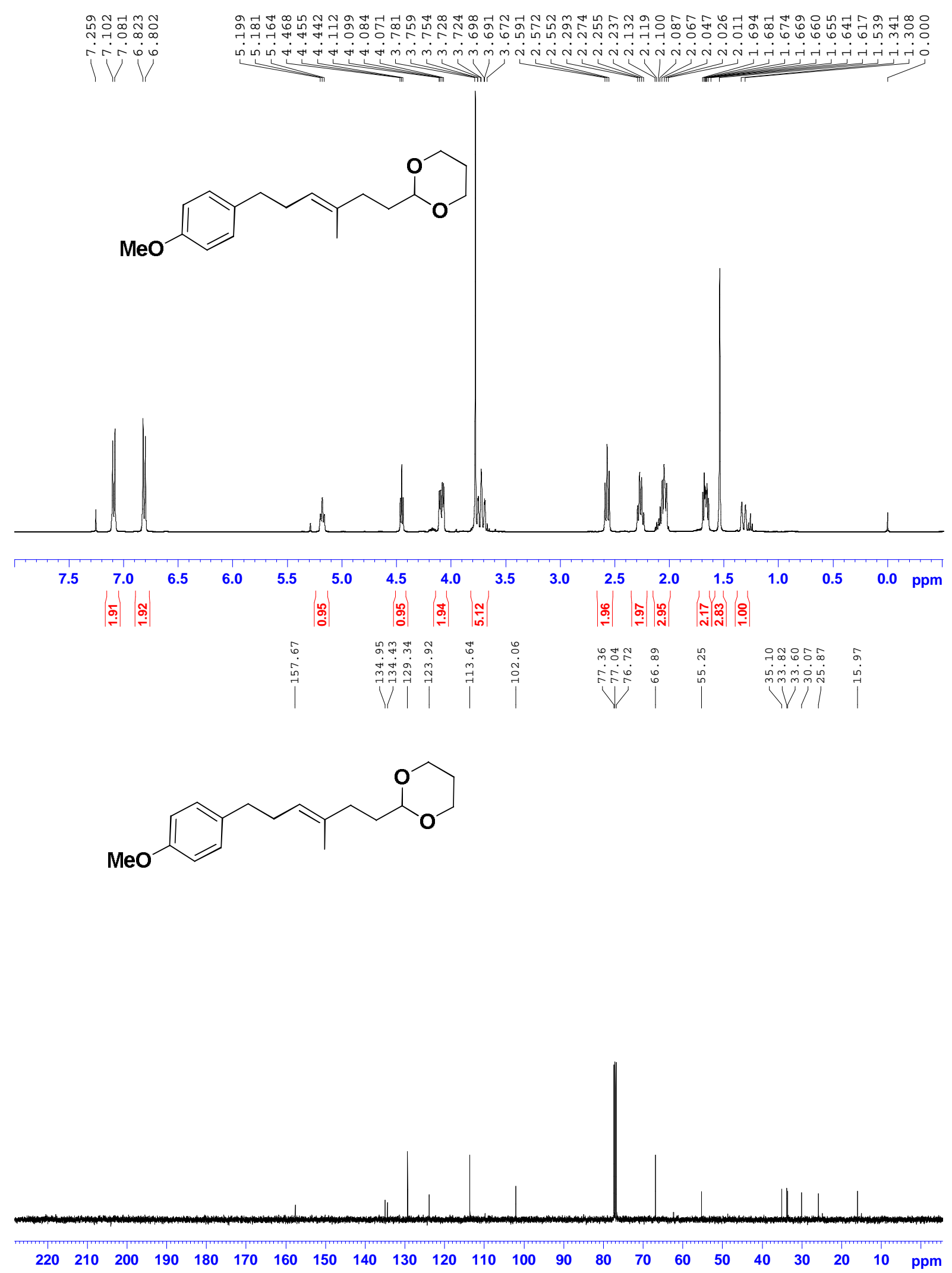


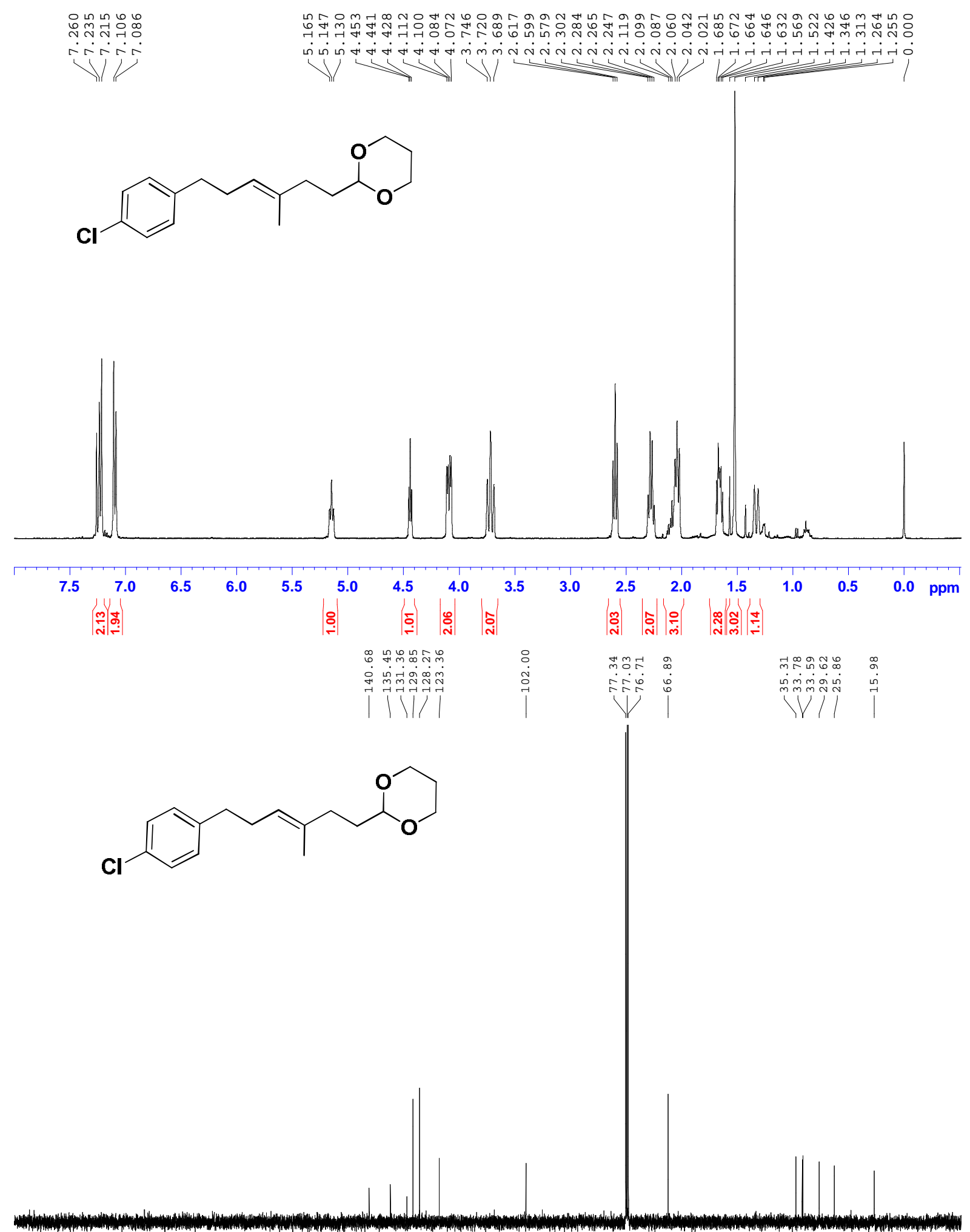

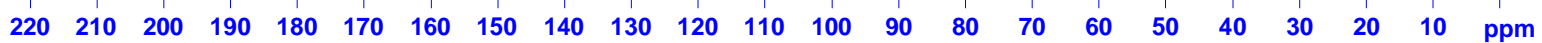


<smiles>CCC/C=C(\C)CC/C=C(\C)CCC1OCCCO1</smiles>
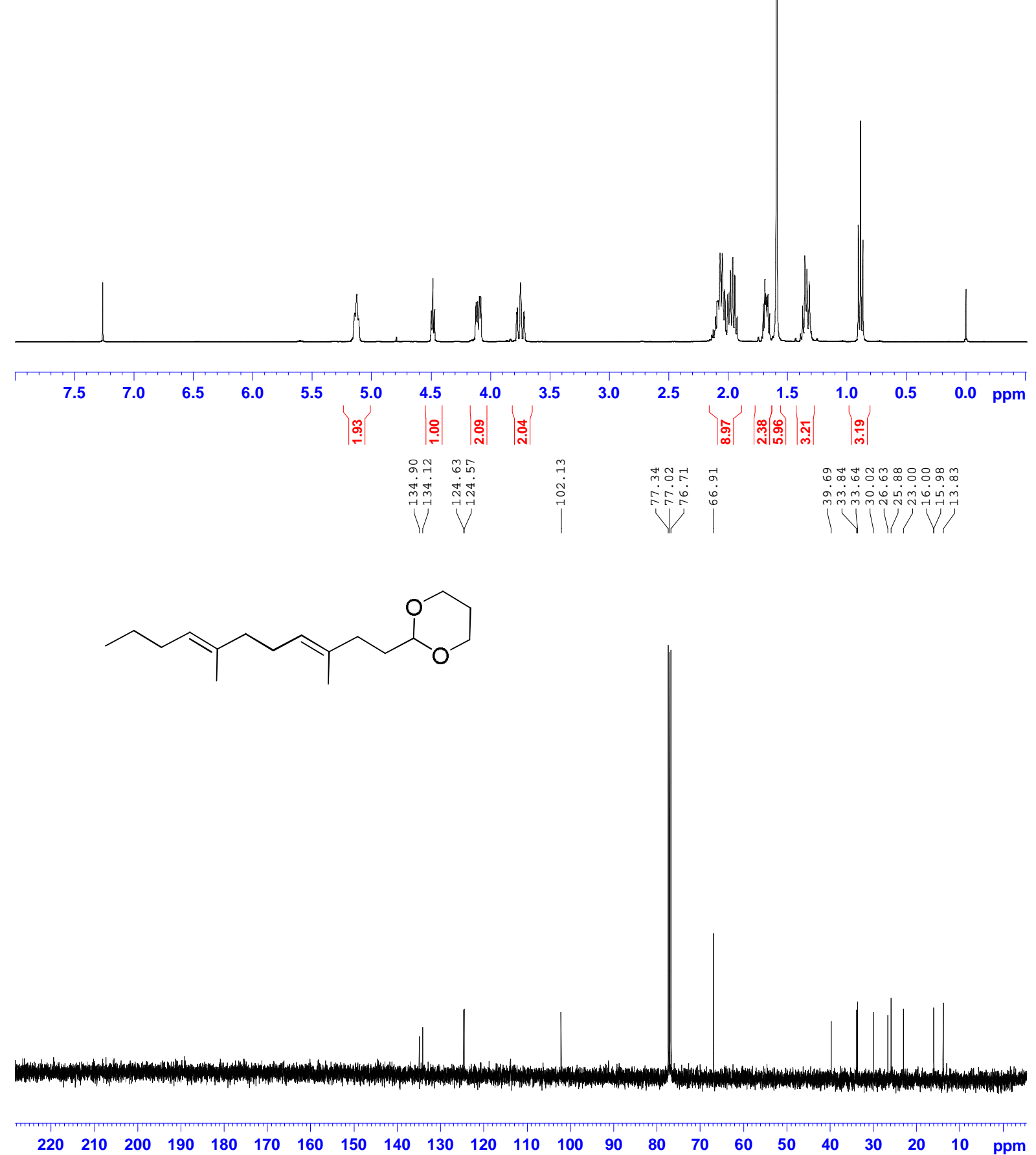


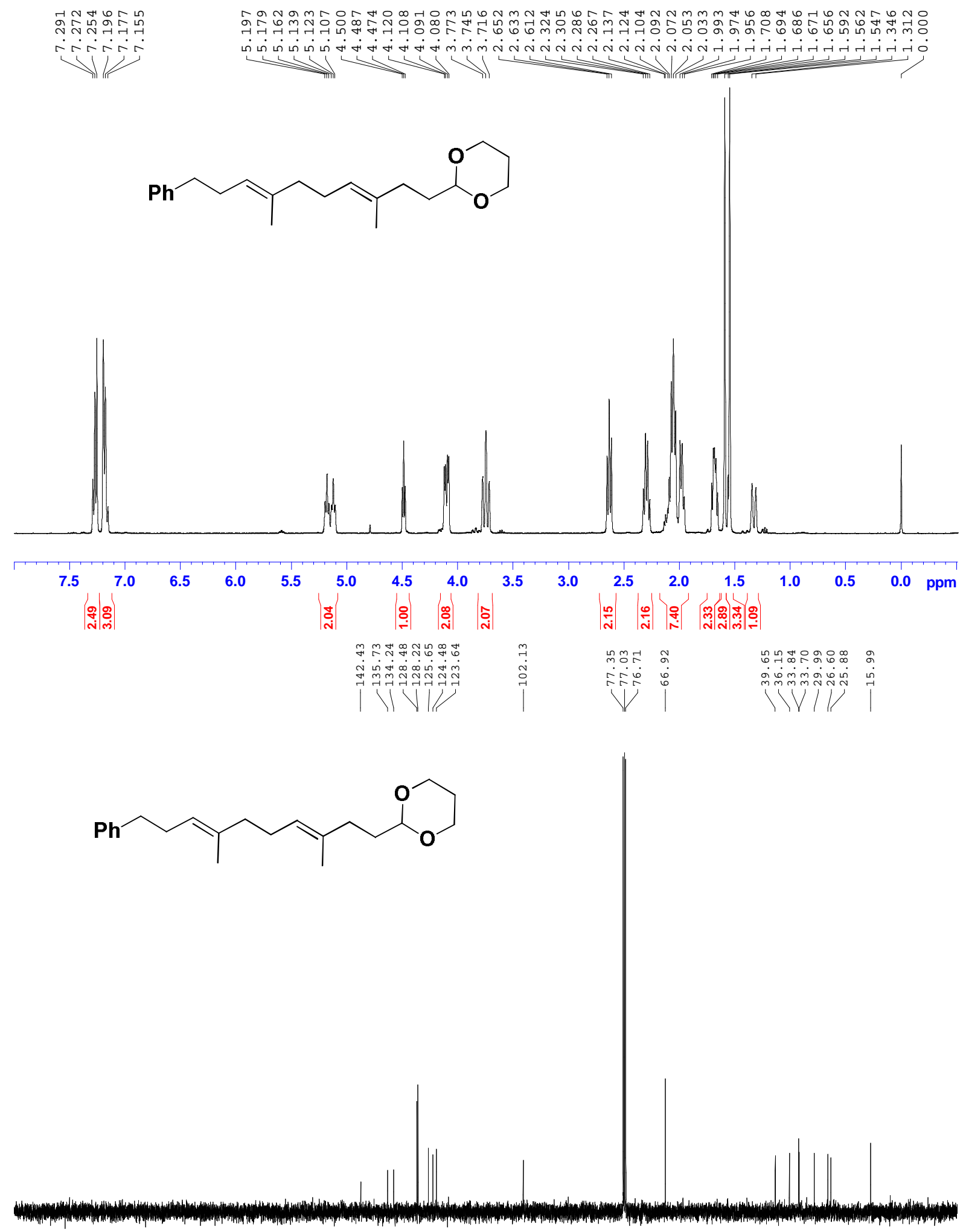

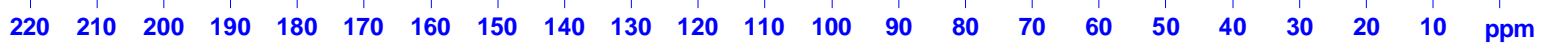




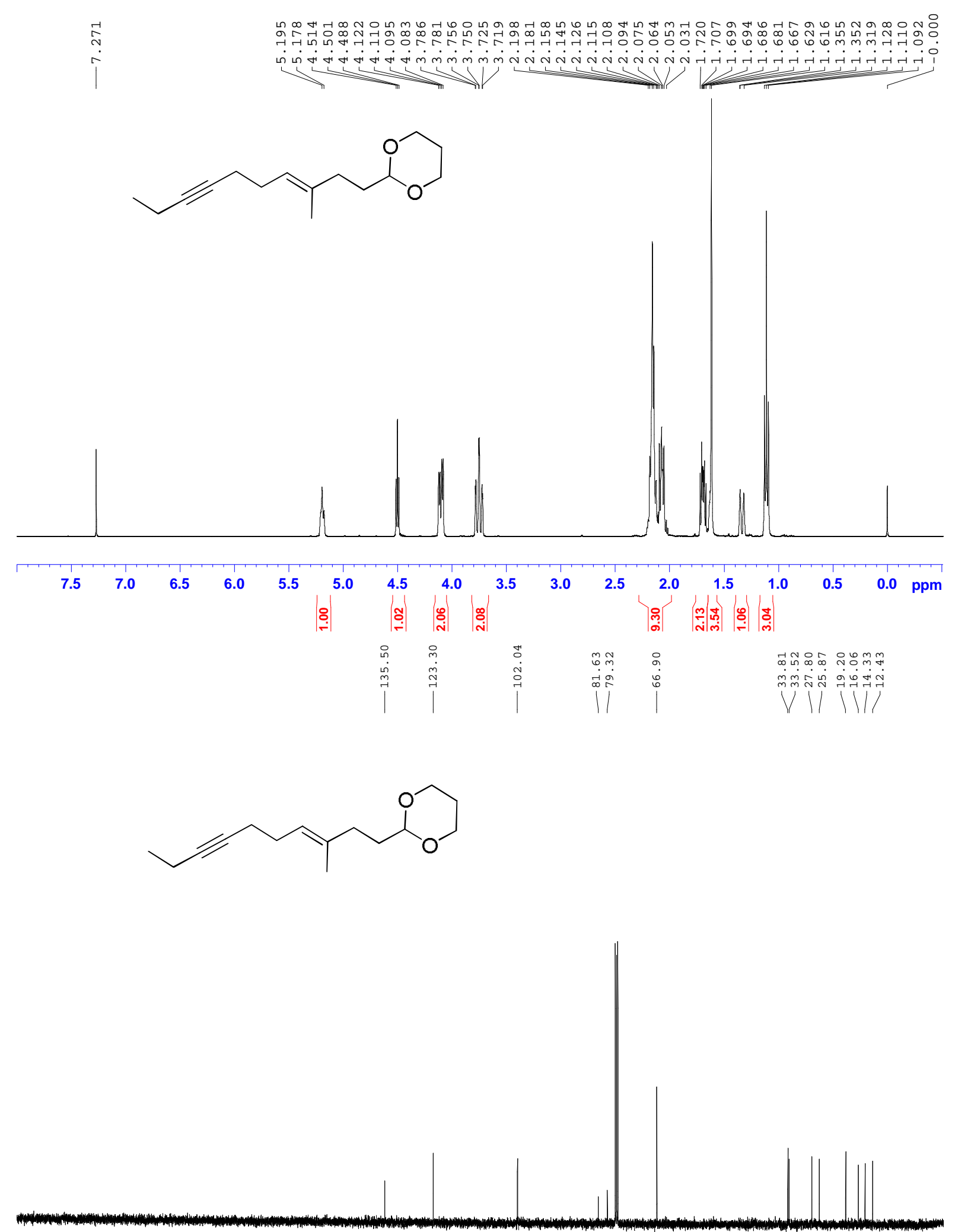

$\begin{array}{lllllllllllllllllllllll}220 & 210 & 200 & 190 & 180 & 170 & 160 & 150 & 140 & 130 & 120 & 110 & 100 & 90 & 80 & 70 & 60 & 50 & 40 & 30 & 20 & 10 & \text { ppm }\end{array}$ 

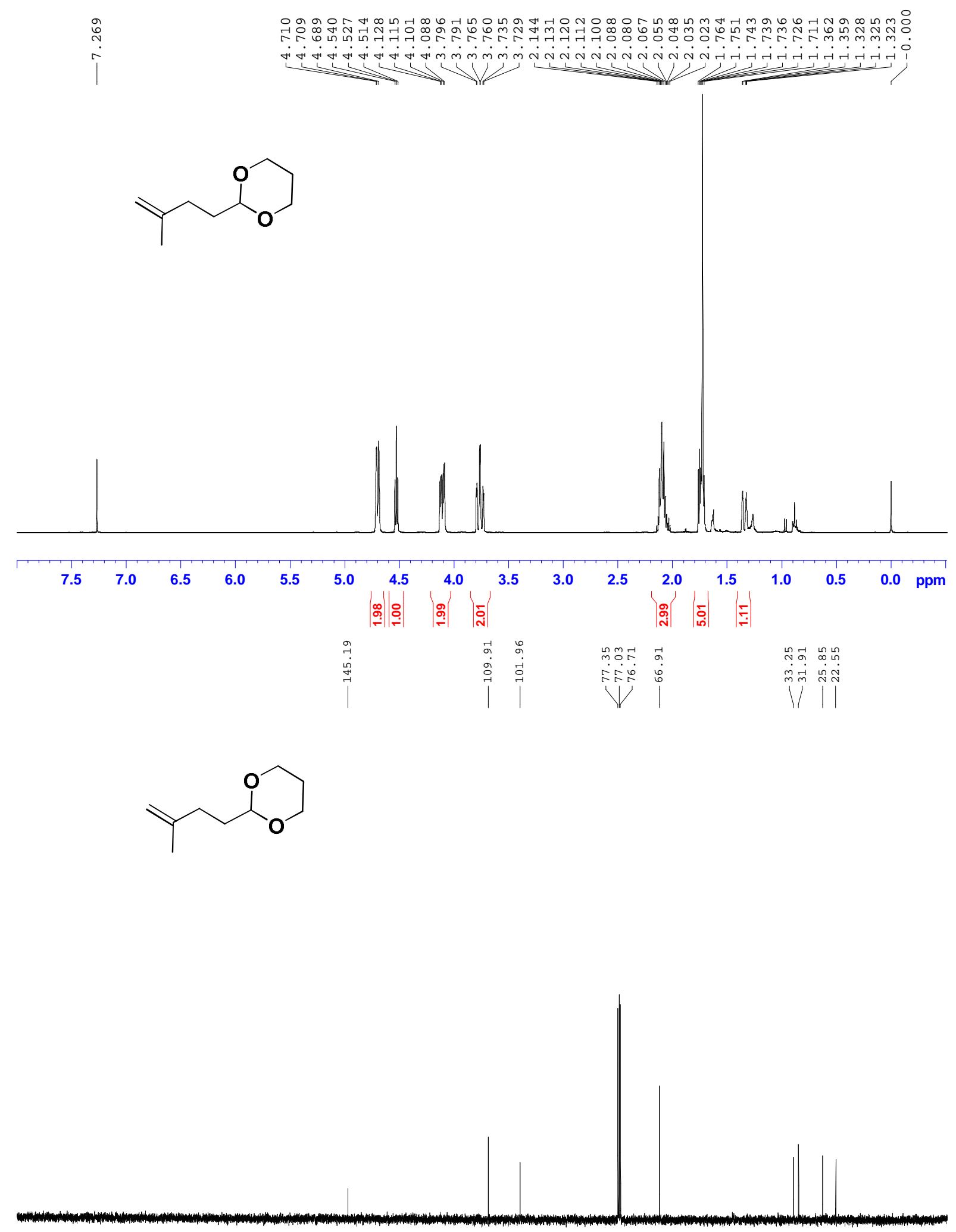

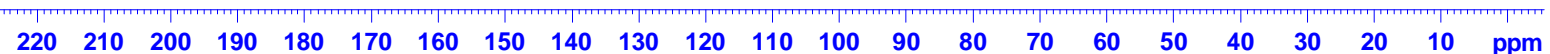




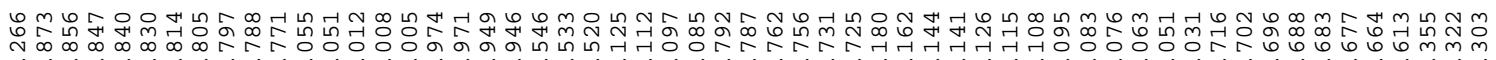

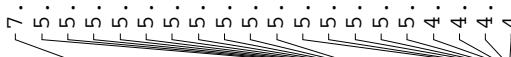<smiles>C=CCCC1OCCCO1</smiles>

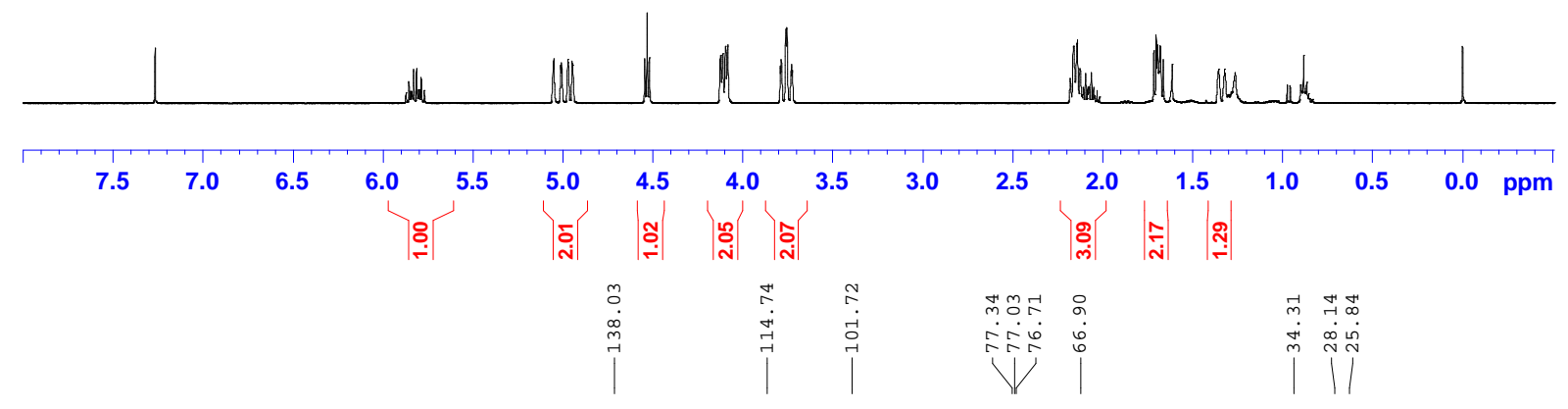<smiles>C=CCCC1OCCCO1</smiles>

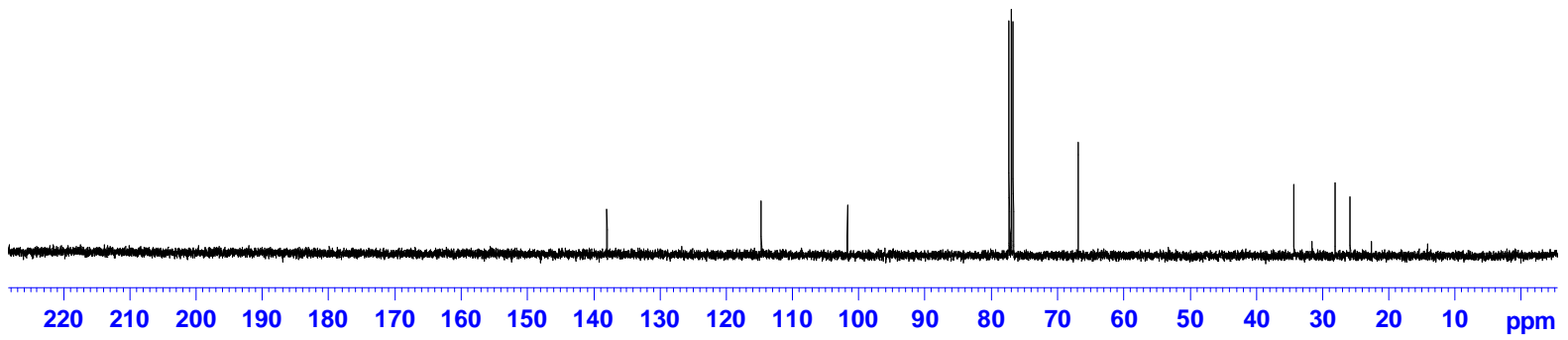




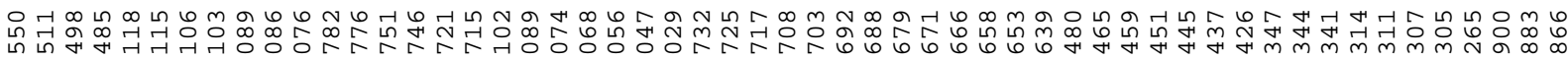

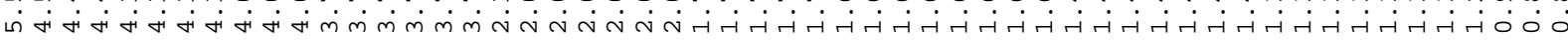<smiles>C1=C(CCC2OCCCO2)CCCCC1</smiles>
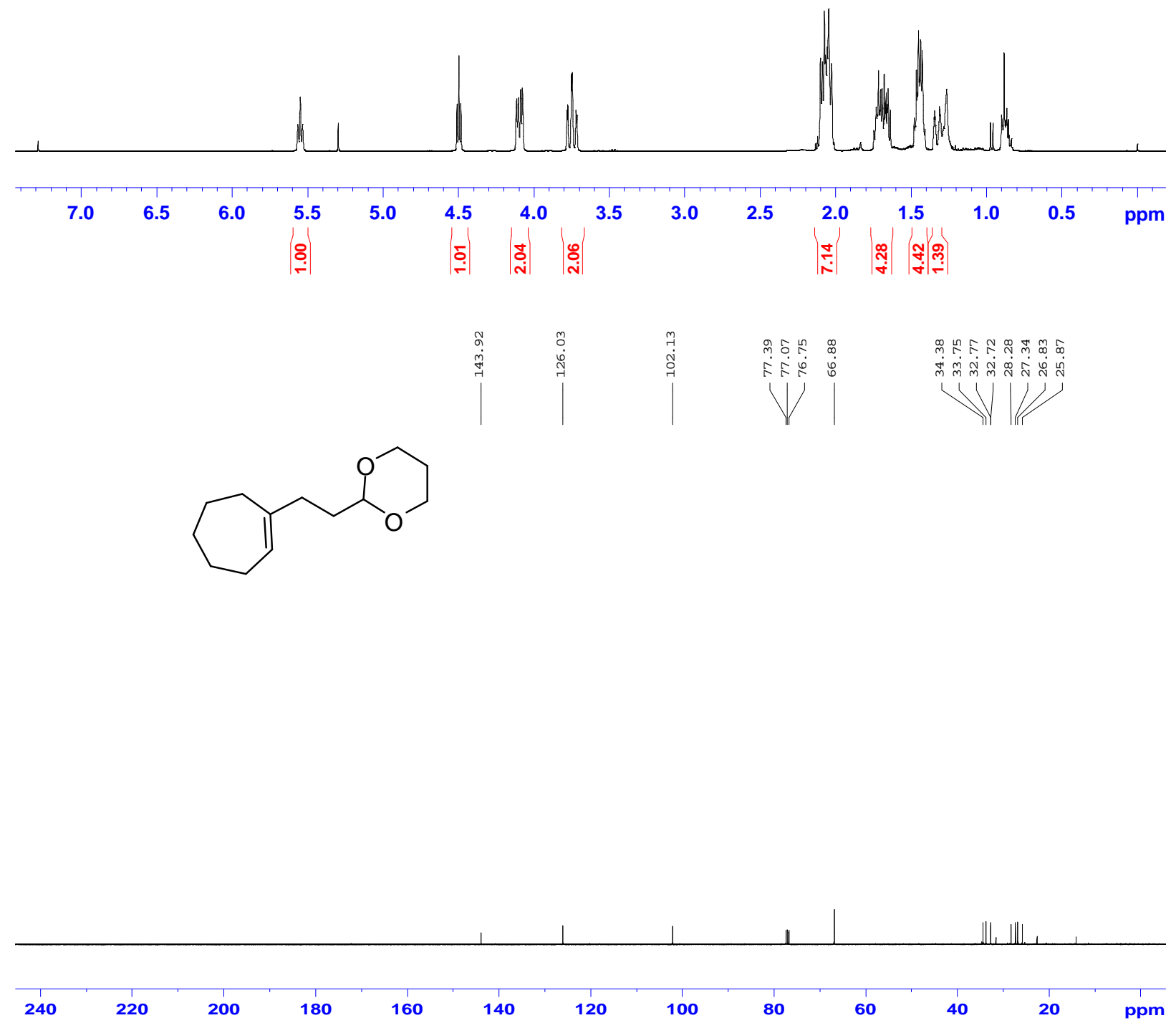
<smiles>C1=C(CCC2OCCCO2)CCCC1</smiles>

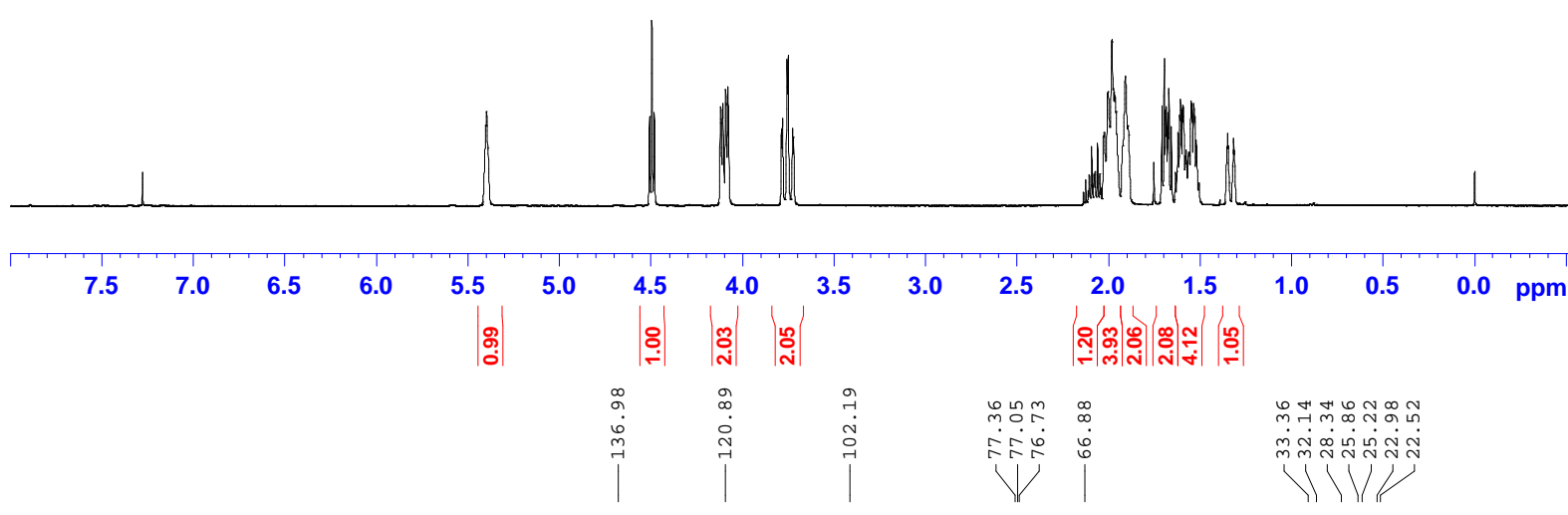<smiles>C1=C(CCC2OCCCO2)CCCC1</smiles>

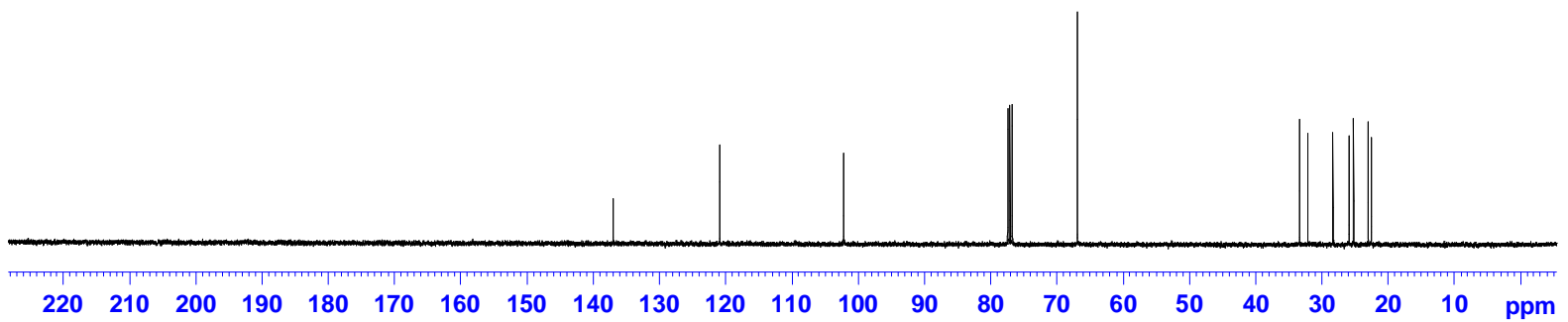


<smiles>C/C(=C\CCc1ccccc1)CC[C@H]1C[C@@H](C)CCO1</smiles>
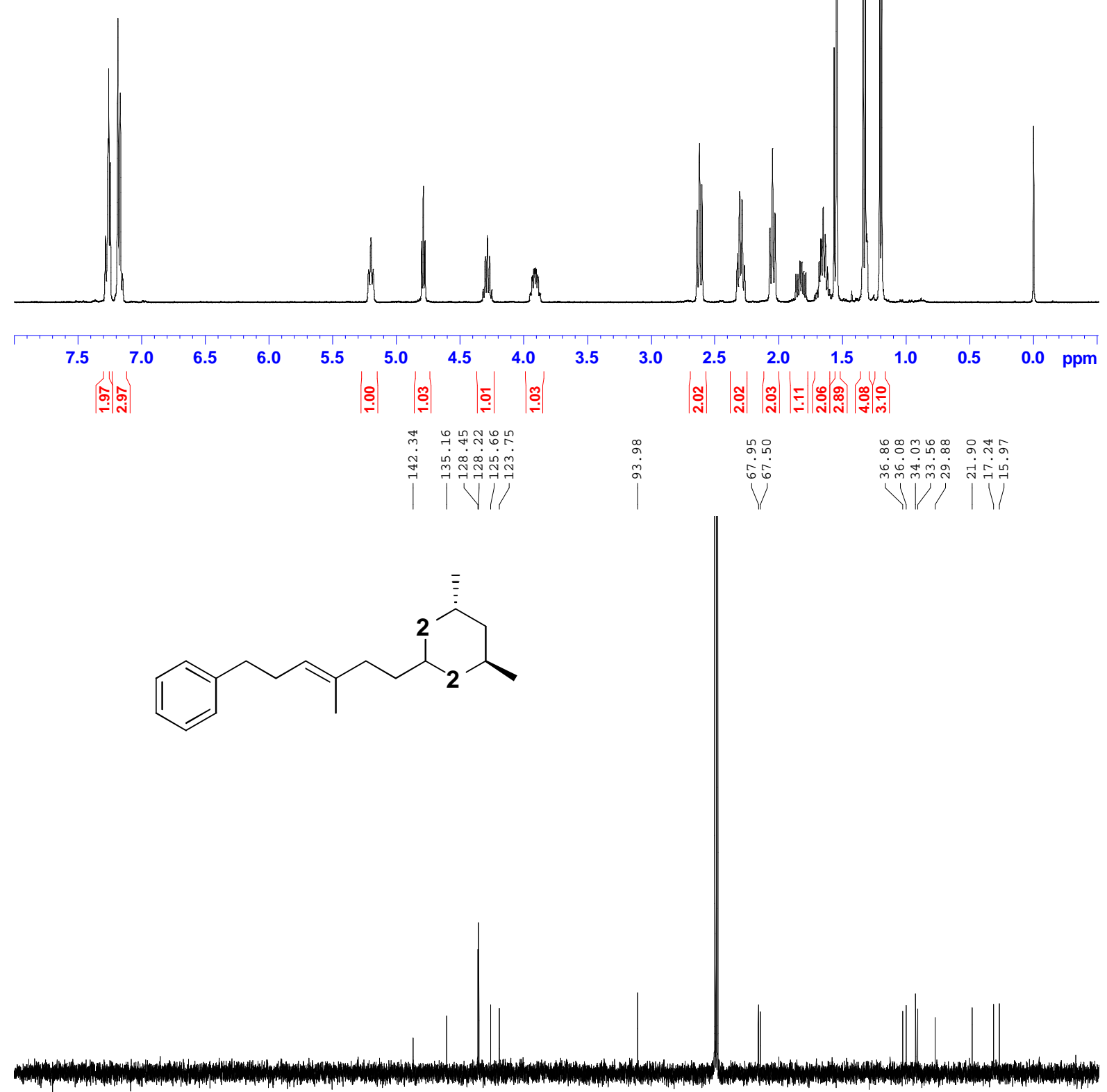

$\begin{array}{lllllllllllllllllllllll}220 & 210 & 200 & 190 & 180 & 170 & 160 & 150 & 140 & 130 & 120 & 110 & 100 & 90 & 80 & 70 & 60 & 50 & 40 & 30 & 20 & 10 & \mathrm{ppm}\end{array}$ 

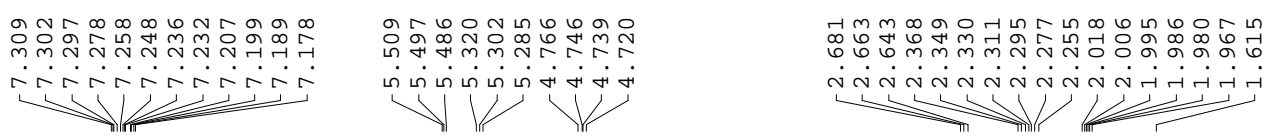

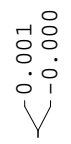
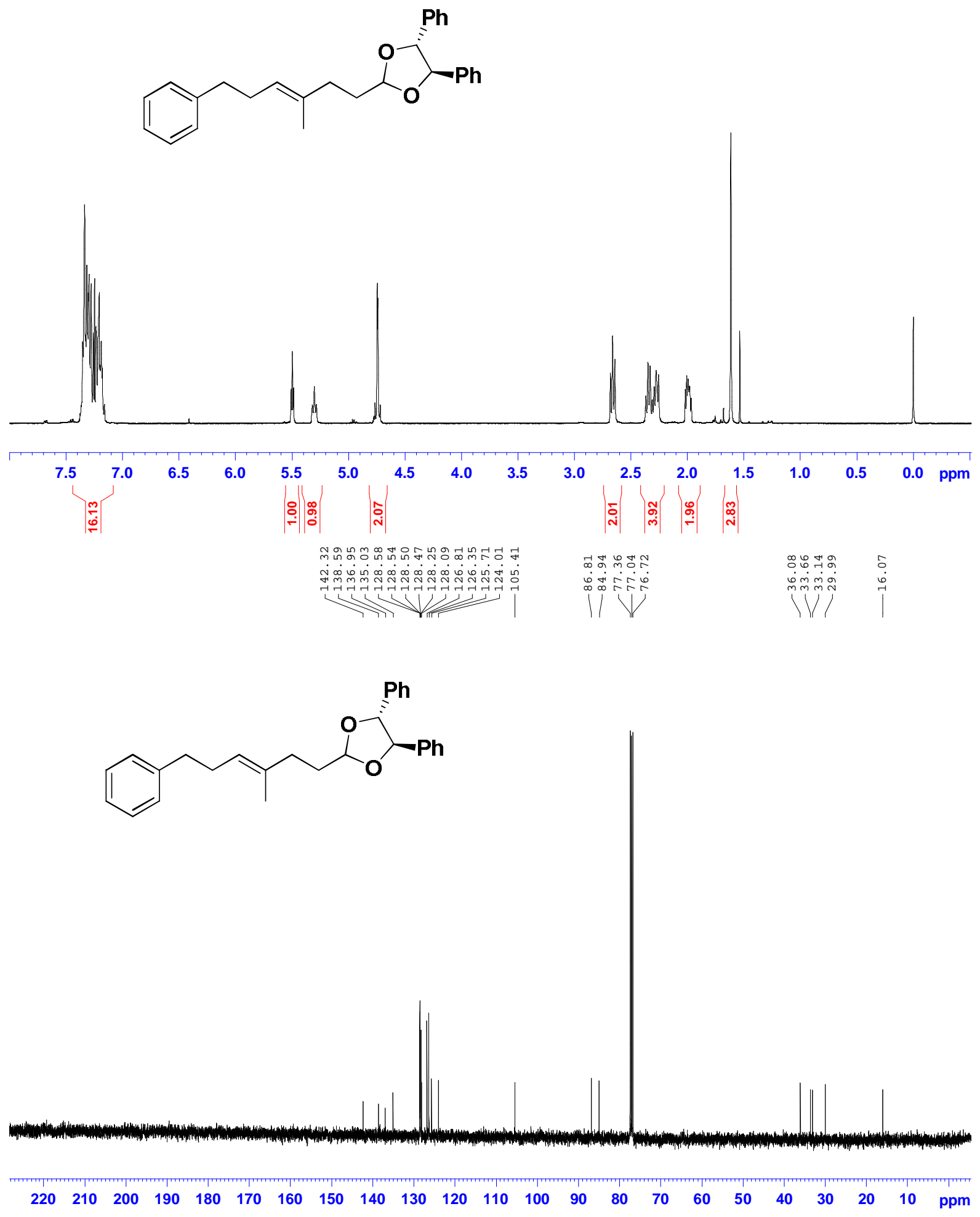

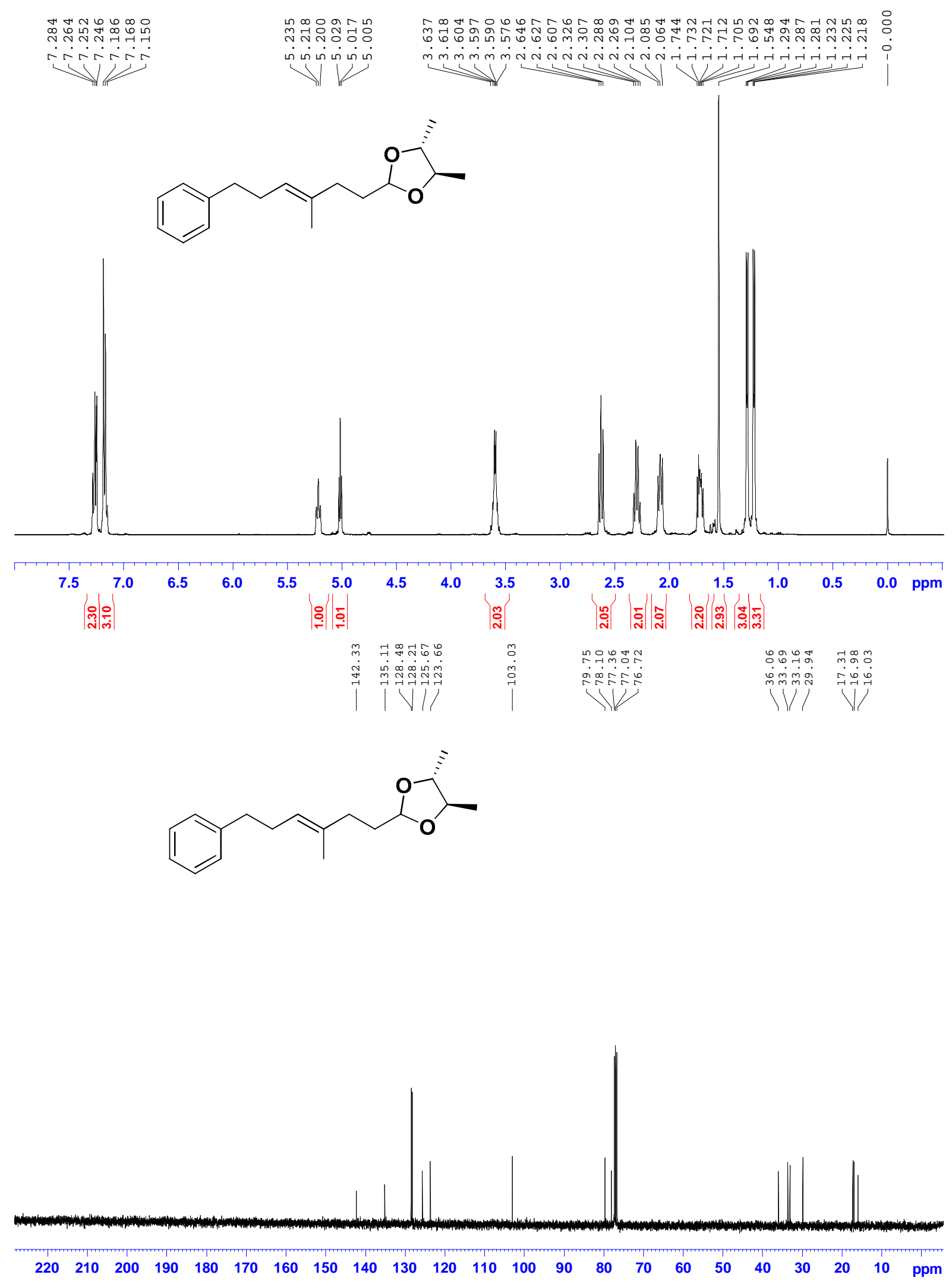

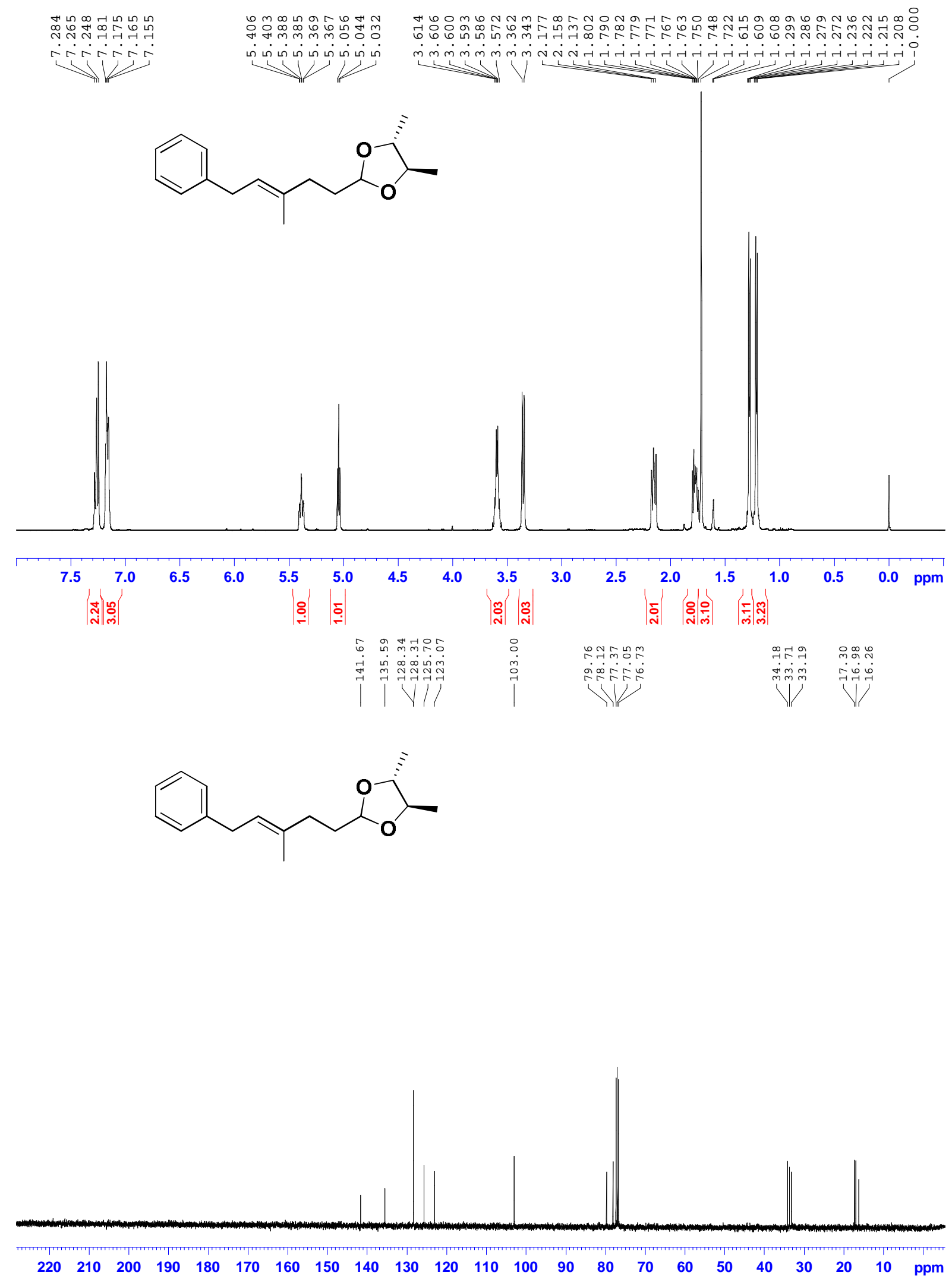

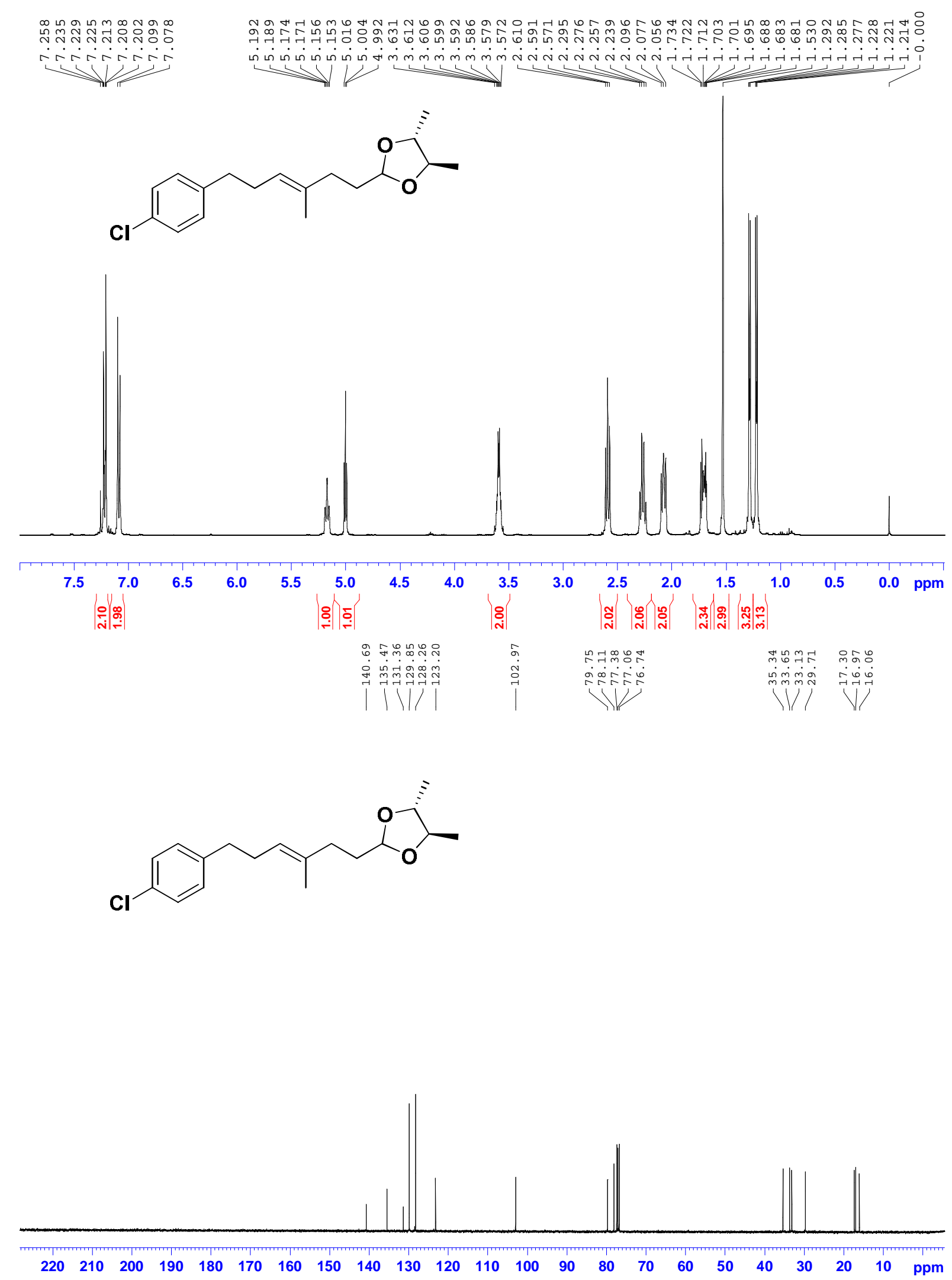

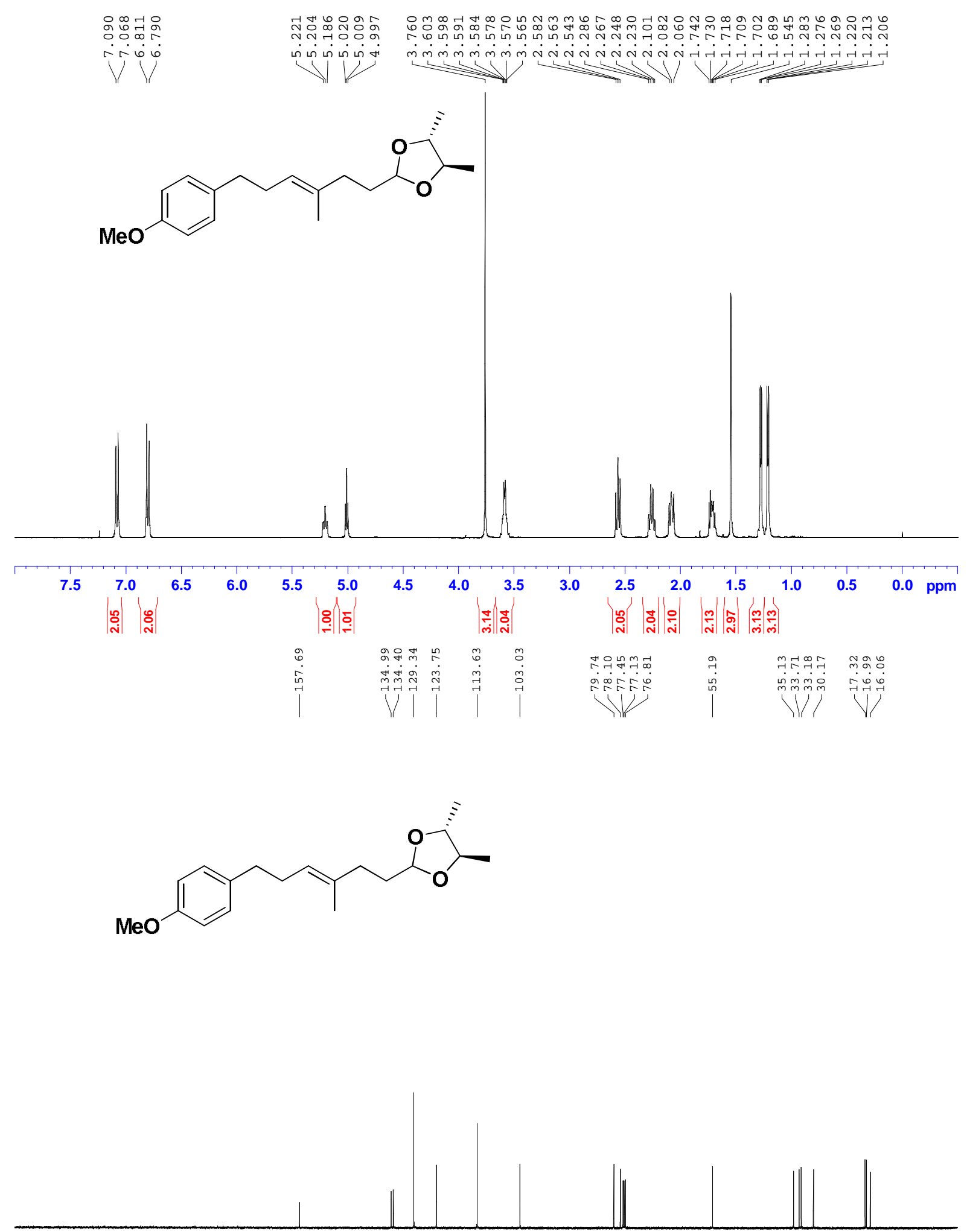

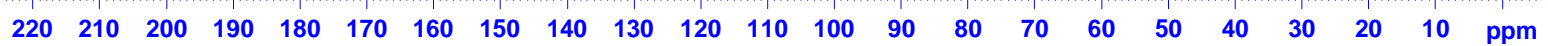



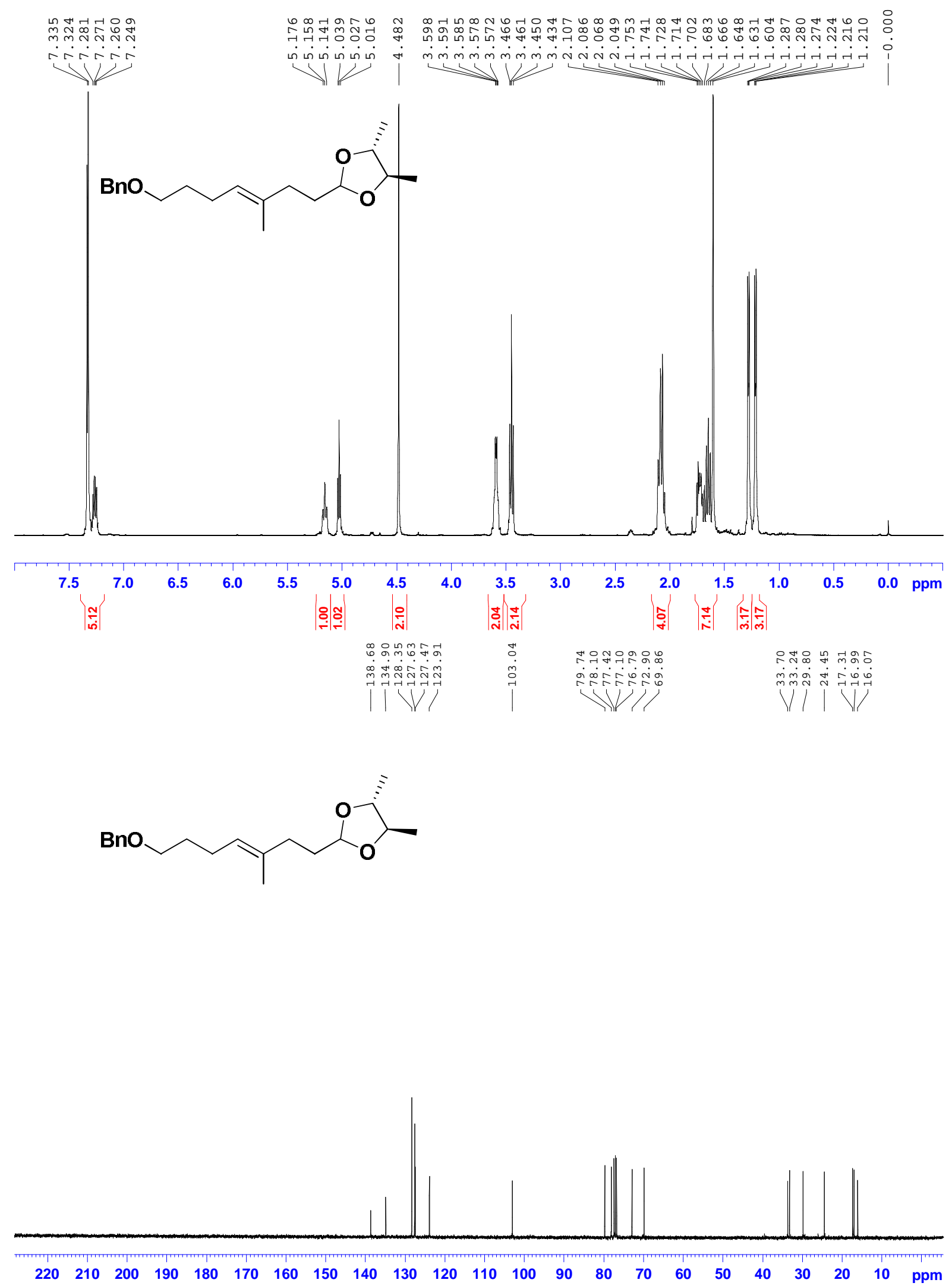
<smiles>C/C(=C\CCc1ccccc1)CCC=O</smiles>

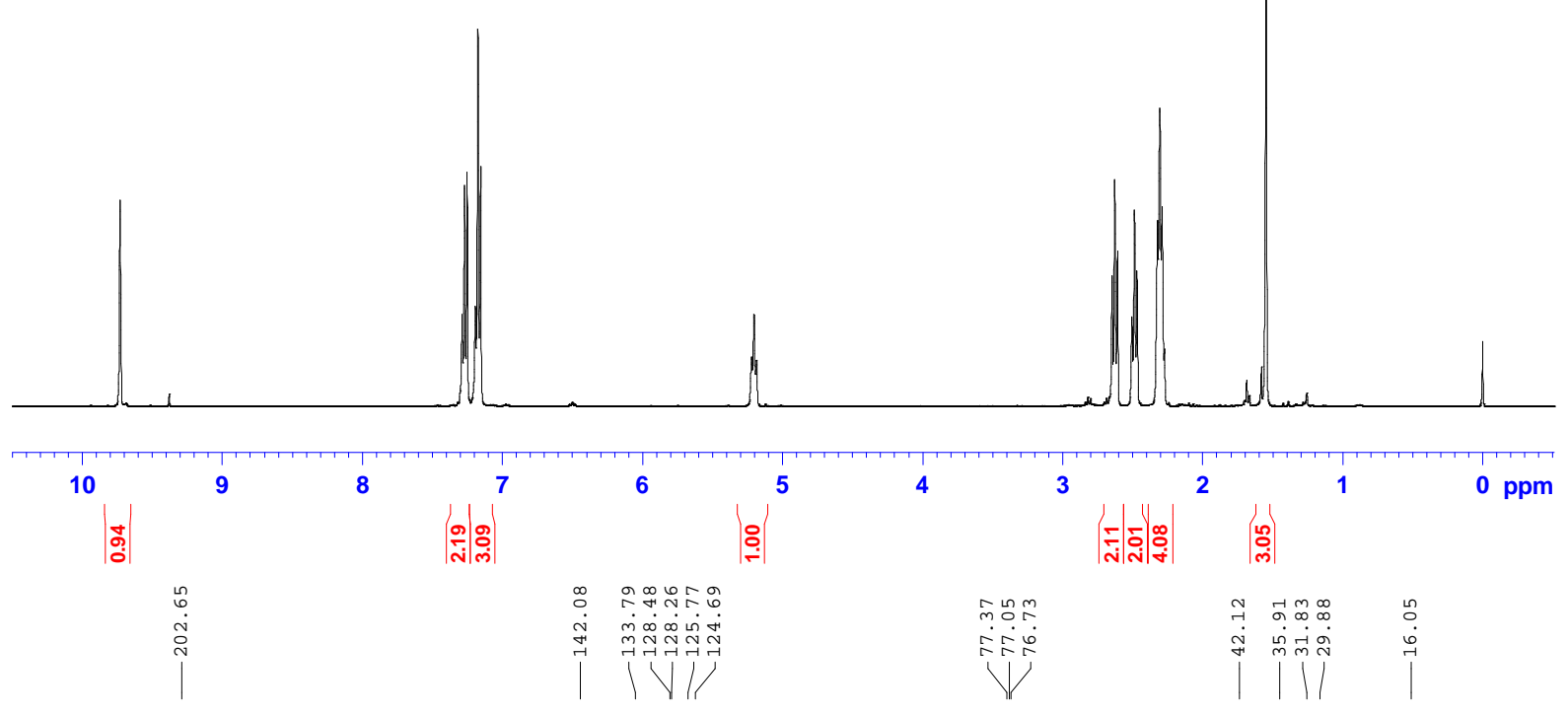<smiles>CC(=CCCc1ccccc1)CCC=O</smiles>

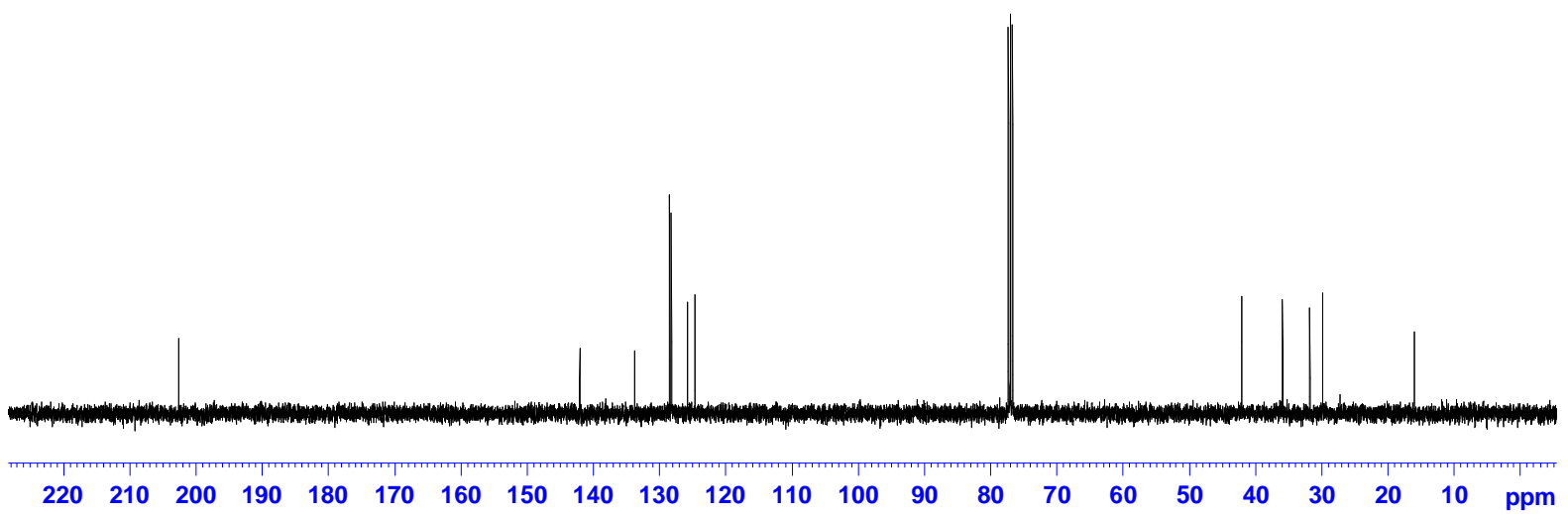




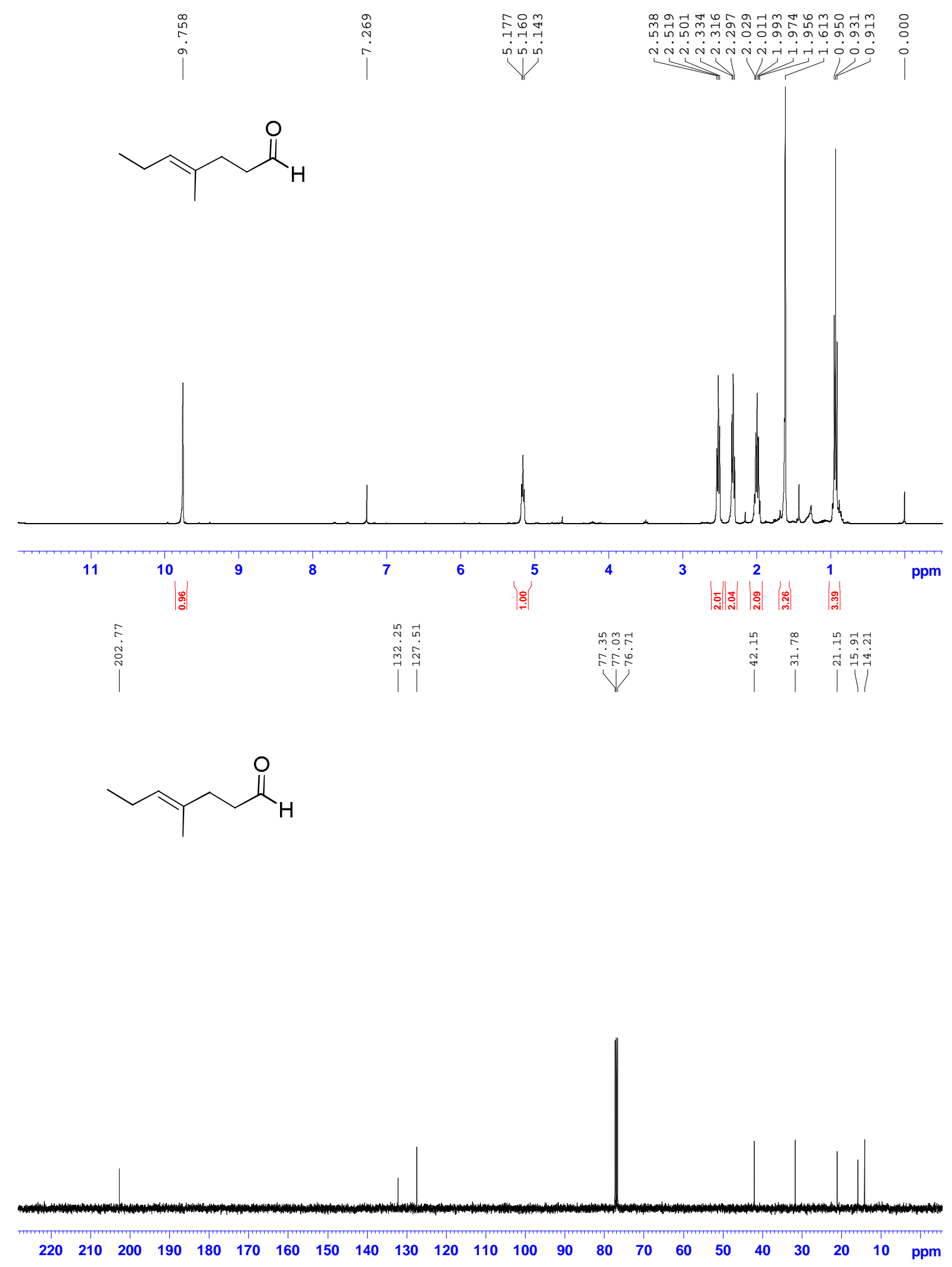




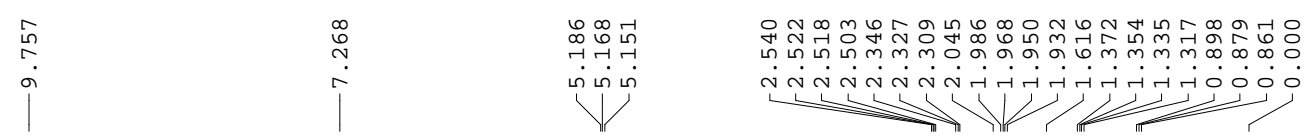<smiles>CCCC=C(C)CCC=O</smiles>
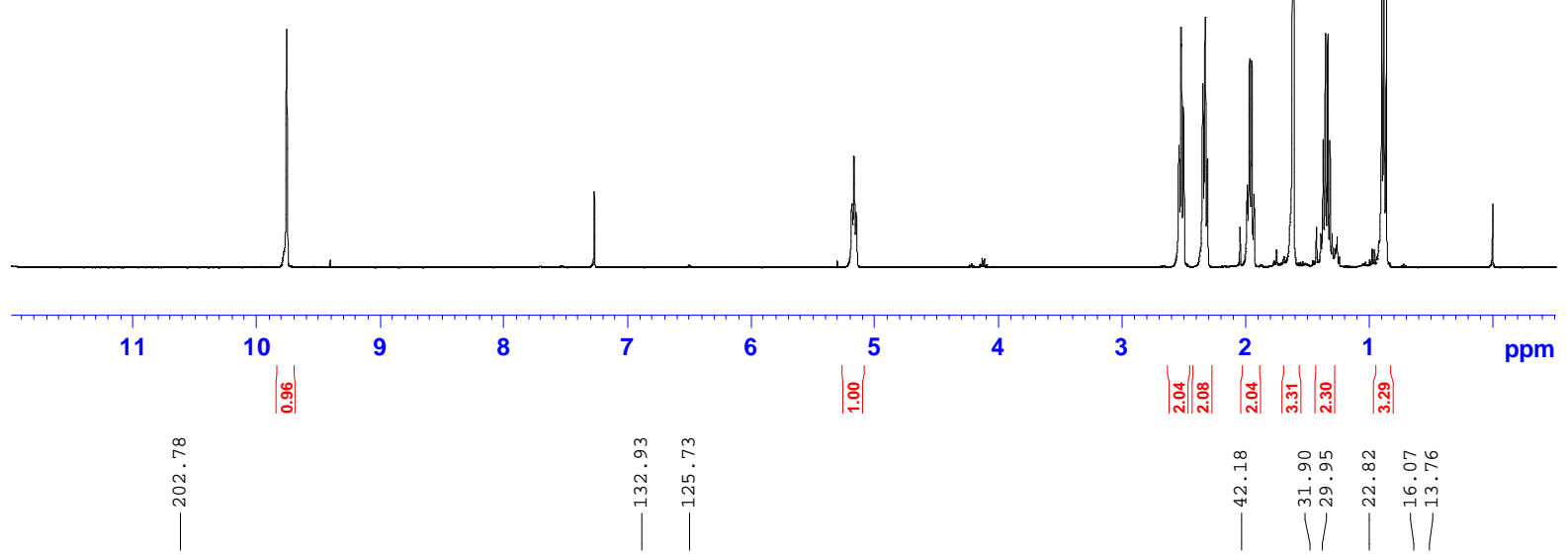<smiles>CCCC=C(C)CCC=O</smiles>

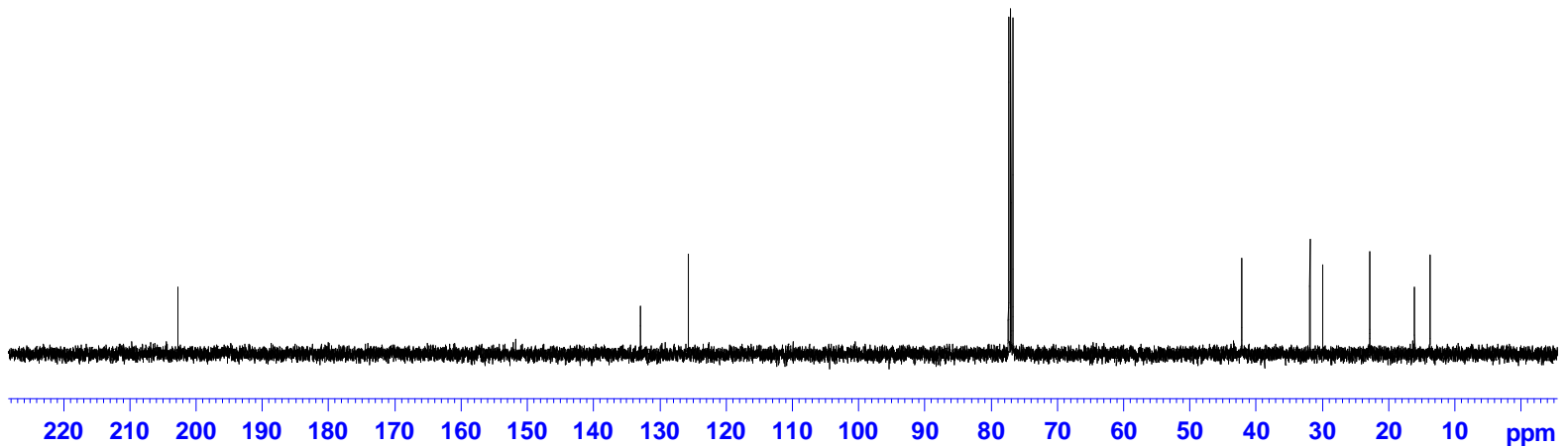




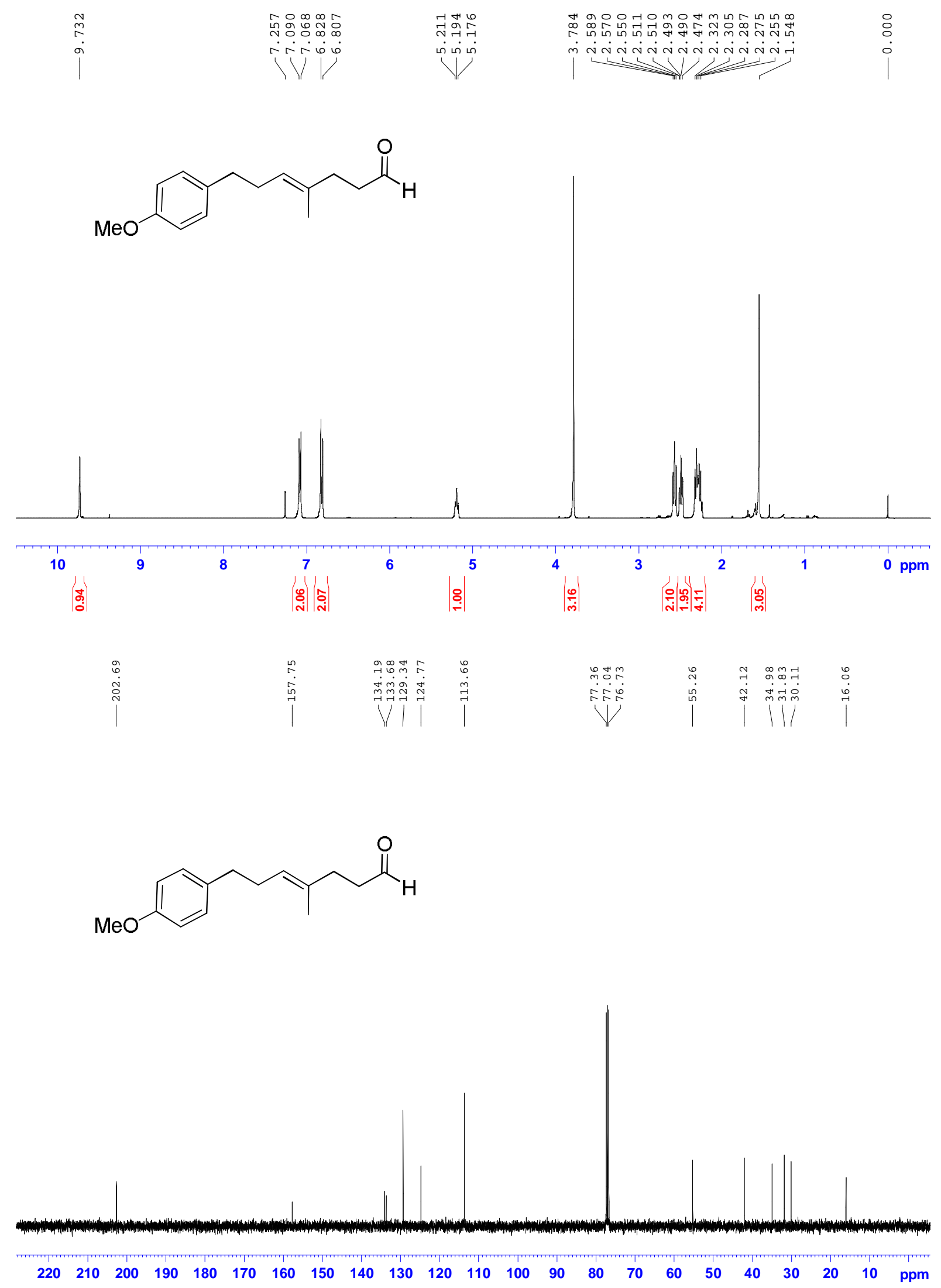



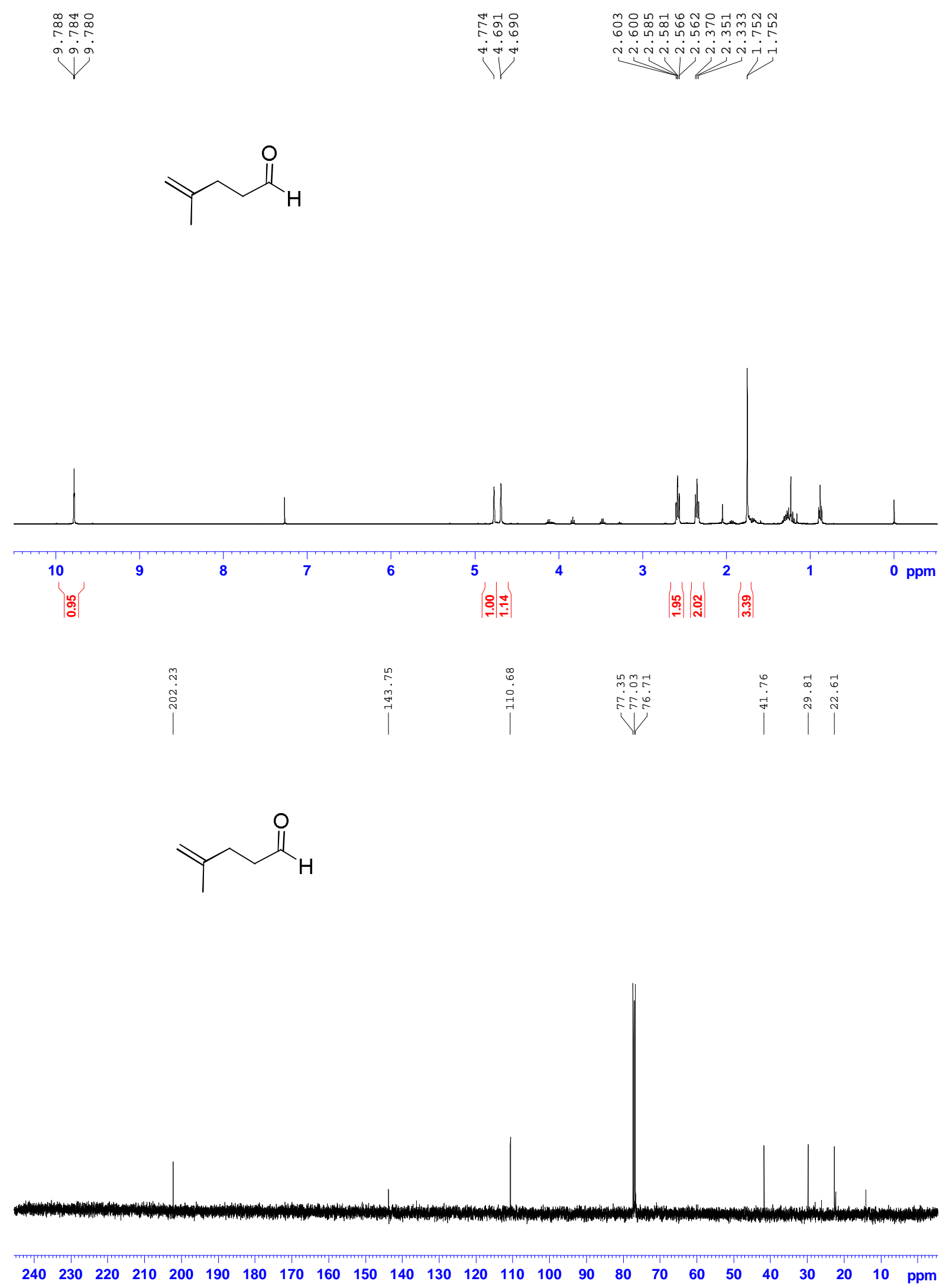


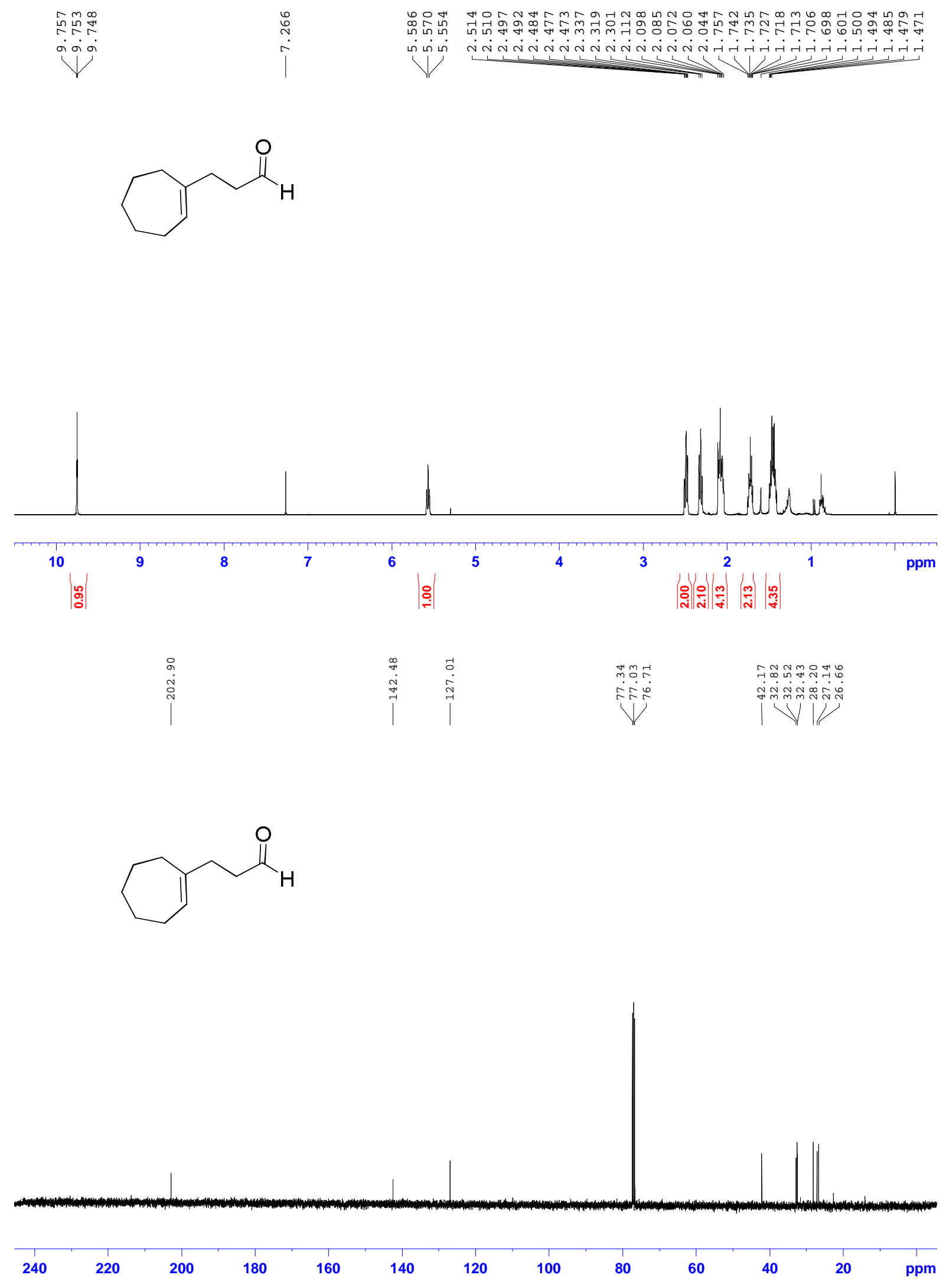

